\title{
Grupos de Lie, ações próprias e a conjectura de Palais-Terng
}

Flausino Lucas Neves Spindola

\author{
DisSERTAÇÃO APRESENTADA \\ $\mathrm{AO}$ \\ Instituto de Matemática e EstatísticA \\ DA \\ Universidade De SÃo Paulo \\ PARA \\ OBTENÇÃO DO TÍTULO \\ $\mathrm{DE}$ \\ Mestre em Ciências
}

Programa de Pós-Graduação em Matemática

Orientador: Prof. Dr. Marcos Martins Alexandrino da Silva

Durante o desenvolvimento deste trabalho o autor recebeu auxílio financeiro do CNPq 


\title{
Grupos de Lie, ações próprias e a conjectura de Palais-Terng
}

\author{
Este exemplar corresponde à redação \\ final da dissertação devidamente corrigida \\ e defendida por Flausino Lucas Neves Spindola \\ e aprovada pela Comissão Julgadora.
}

Banca Examinadora:

- Prof. Dr. Marcos Martins Alexandrino da Silva - IME-USP.

- Prof. Dr. Rosa Maria dos Santos Barreiro Chaves - IME-USP.

- Prof. Dr. Luiz Amancio Machado de Sousa Junior - UNIRIO. 
À minha mãe, Ana

e irmãs, Valéria e Ana Carolina. 


\section{Agradecimentos}

Nesse momento, fazendo um resumo de minha trajetória, vários momentos e pessoas se passam à mente. Mas certamente algumas delas se tornam imprescindíveis.

A começar pelo seio familiar, o quanto eu puder agradecer e honrar o nome de minha mãe, Ana Neves, ainda assim seria pouco. Mas na limitação das letras vale a pena prestar uma homenagem, que seja breve, a essa mulher que me integrou ao mundo e me ensinou as boas lições que permeiam minha existência, de forma muito dócil e sempre repleta de amor.

Não posso esquecer de minhas irmãs, Ana Carolina e Valéria, as quais me acompanham desde o meu nascimento. Elas me dão suporte emocional para a vida, e são essenciais em meu crescimento como pessoa. Irmãs, sou muito grato por todas as vezes que se preocuparam comigo, me apoiaram e estimularam.

Mas um projeto de mestrado não se faz sem um grande mestre na guia. E certamente tive a sorte de encontrar uma pessoa magnífica para me conduzir. Dizer que Marcos é meu orientador de mestrado é pouco, porque ele o é para a vida. Sua preocupação com os alunos extrapola o ambiente acadêmico e o trabalho em si, mas preza por uma formação completa. Jamais esquecerei as horas de conversas sobre perspectivas futuras e o incentivo dado à busca pelo conhecimento. Marcos suas lições, sejam de geometria ou de vida, sempre carregarei comigo.

Bem, não posso esquecer dos amigos e demais pessoas com as quais convivemos no ambiente da universidade. Aldair e Gustavo são grandes companheiros de residência, os quais considero como família; Eduardo, um bom amigo. Vou evitar fazer uma lista de nomes, mas agradeço aqui a todos os colegas e amigos que, de forma direta ou indireta, estiveram comigo em algum momento. Vocês todos são muito importantes pra mim! 


\section{Resumo}

Esta dissertação tem dois objetivos.

Nosso primeiro objetivo é apresentar alguns aspectos da teoria dos grupos de Lie e ações próprias, baseado em aulas dadas por M. Alexandrino

Nosso segundo objetivo e rever a prova da conjectura de Terng e Palais dada por Alexandrino [A]. Este teorema nos garante que folheações riemannianas singulares com distribuição normal é uma folheação riemanniana singular com seções.

Adaptamos a demonstração para o caso particular das ações isometricas.

Palavras-chave: Grupos de Lie, Ações Próprias, Conjectura de Palais-Terng. 


\section{Abstract}

The aim of this work is twofold.

Our first goal is to present some aspects of the theory of Lie group and proper action based on lectures given by M. Alexandrino.

The second goal is to review the proof of the conjecture of Terng and Palais given by Alexandrino [A]. This theorem assure us that a singular riemannian foliation with integrable normal distributions is is a singular riemannian foliation with section.

We adapt the proof for the particular case of isometric action.

Keywords: Lie Groups, Proper Actions, Palais-Terng Conjecture. 


\section{Sumário}

1 Resultados Básicos de Grupos de Lie $\quad 3$

1.1 Grupos e Álgebras de Lie . . . . . . . . . . . . . . . . . . . 3

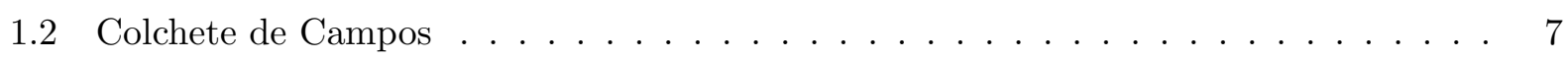

1.3 Campos Invariantes à Esquerda . . . . . . . . . . . . . . . . . 9

1.4 Subgrupos e Homomorfismos de Lie . . . . . . . . . . . . . . . . . . . 12

1.5 Aplicação Exponencial e Representação Adjunta . . . . . . . . . . . . . . . . . . 19

1.6 Subgrupos Fechados . . . . . . . . . . . . . . . . . . 22

1.7 Fatos de Geometria Riemanniana . . . . . . . . . . . . . . . . 26

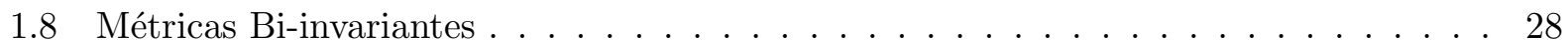

1.9 Forma de Killing . . . . . . . . . . . . . . . . . . . . . . 32

1.10 Decomposição em Ideais Simples ． . . . . . . . . . . . . . . . . . . 34

2 Ações Próprias $\quad 39$

2.1 Definições e Resultados Iniciais . . . . . . . . . . . . . . . . . . . . 39

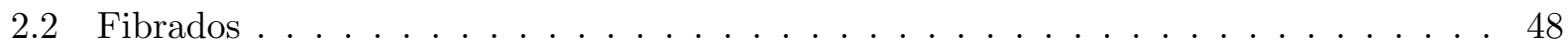

2.3 Existência de Slice . . . . . . . . . . . . . . . . . . . . 57

2.4 Fibrados Associados . . . . . . . . . . . . . . . . . . 61

2.5 Vizinhança Tubular . . . . . . . . . . . . . . . . . . . . . . . . . . . . . . . . 62

2.6 Ações Isométricas . . . . . . . . . . . . . . . . . . . . . . . . 67

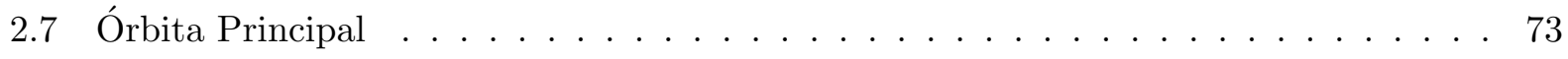

2.8 Campos de Killing . . . . . . . . . . . . . . . . . . . . . 76

2.9 Tipos de Órbita . . . . . . . . . . . . . . . . . . . 80

3 A Conjectura de Terng-Palais $\quad \mathbf{8 5}$

3.1 Fatos sobre Imersões Riemannianas . . . . . . . . . . . . . . . . . . . . . . 85

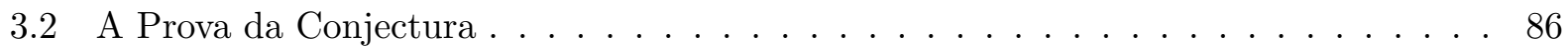

3.2 .1 Demonstração do Teorema $3.8 \ldots \ldots$. . . . . . . . . . . . . . 90

$\begin{array}{ll}\text { Referências Bibliográficas } & 93\end{array}$

$\begin{array}{ll}\text { Referências Bibliográficas } & 93\end{array}$ 


\section{Introdução}

Apresentamos aqui um texto introdutório auto-suficiente sobre grupos de Lie e ações, no intuito de organizar um instrumental teórico importante para compreender e demonstrar o teorema final deste texto (a conjectura de Palais-Terng). Algumas vezes, alguns conceitos e teoremas importantes que exigiriam uma abordagem mais geral serão utilizados, mas para evitar o desvio de nosso foco central e prolongar demasiadamente a dissertação, nos ocuparemos em indicar referências onde tais conceitos podem ser elucidados para eventual consulta do leitor.

Os dois primeiros capítulos desta dissertação são baseados em notas de aula da disciplina "Introdução aos Grupos de Lie e Ações Próprias" ministrada no primeiro semestre de 2006 pelo professor Marcos Alexandrino no IME/USP. Em nosso primeiro capítulo, buscamos reunir as definições e resultados da teoria dos grupos e álgebras de Lie de maneira sucinta e compreensível para um leitor a nível de mestrado, também apresentando relações importantes que podem ser feitas ao pensar nos subgrupos e subálgebras de Lie. A disposição das seções permite um estudo cuidadoso das proposições principais e finalizamos com importante resultado sobre decomposição de álgebras e grupos de Lie.

O segundo capítulo se preocupa em introduzir as ações de um grupo de Lie em uma variedade. Neste capítulo é que tratamos das ações isométricas próprias bem como dos espaços de órbitas e slice tão necessárias para o trabalho no capítulo 3 .

No capítulo 3 finalmente vamos atacar o problema proposto por Palais-Terng, que garante a existência de uma seção imersa completa totalmente geodésica sempre ortogonal às órbitas de uma ação isométrica própria caso a distribuição normal às órbitas seja integrável. Nesta demonstração, vamos reunir todo o conhecimento acumulado sobre ações de grupos de Lie na tentativa de demonstrar um resultado recente. Para tal, faremos uma adaptação da prova de Alexandrino ( [A]) ao caso particular de órbitas de uma ação isométrica própria. 


\section{Capítulo 1}

\section{Resultados Básicos de Grupos de Lie}

Este capítulo consiste em um texto claro e objetivo sobre a teoria dos grupos de Lie. Os conceitos principais dessa teoria são aqui apresentados e as propriedades enunciadas e demonstradas.

De início, vamos introduzir as definições de grupos de Lie e álgebras de Lie, estabelecendo condições para construir álgebra de Lie a partir de grupo de Lie (através de campos invariantes à esquerda) e admitindo o terceiro teorema de Lie que nos garante a existência de um grupo de Lie conexo para uma álgebra de Lie dada.

Um estudo importante de subgrupos e subálgebras de Lie é realizado na seção 1.4, o que a torna rica em proposições e teoremas importantes sobre homomorfismos de grupos e de álgebras de Lie. Estudamos em seguida a exponencial de Lie e sua relação com os conceitos de adjunta e adjunta pequena. Finalizamos com o estudo das métricas bi-invariantes e mostrando um importante resultado de decomposição de grupos de Lie por meio do qual estabelecemos condições para a existência de uma única métrica bi-invariante.

\subsection{Grupos e Álgebras de Lie}

Procuramos nesta seção introduzir de forma clara os conceitos de grupos de Lie e álgebras de Lie. Para isto, iniciamos definindo variedade suave e depois dando a ela a estrutura de grupo necessária para torná-la um grupo de Lie. Exibiremos alguns exemplos importantes na compreensão da definição e mostraremos o primeiro resultado interessante desse capítulo que dá estrutura de grupo de Lie a uma subvariedade mergulhada de grupo de Lie.

Definição 1.1 (Variedade). Uma variedade suave (analítica) $M$, de dimensão $m$ (usualmente denotada por $M^{m}$ ), é um espaço topológico Hausdorff com base enumerável munido de uma família de homeomorfismos

$$
\varphi_{\alpha}: \mathcal{U}_{\alpha} \longrightarrow \mathcal{V}_{\alpha}
$$

de abertos $\mathcal{U}_{\alpha}$ de $M$ em abertos $\mathcal{V}_{\alpha}$ de $\mathbb{R}^{m}$ satisfazendo as propriedades:

1) $M=\cup_{\alpha} \mathcal{U}_{\alpha}$

2) Se $\mathcal{W}=\mathcal{U}_{\alpha} \cap \mathcal{U}_{\beta} \neq \emptyset$, então

$$
\varphi_{\beta} \circ \varphi_{\alpha}^{-1}: \varphi_{\alpha}(\mathcal{W}) \longrightarrow \varphi_{\beta}(\mathcal{W})
$$


é suave (analítica)

3) A família $\left\{\left(\mathcal{U}_{\alpha}, \varphi_{\alpha}\right)\right\}$ é maxima com relação às condições acima.

Observação 1.2. Algumas denominações especiais são dadas aos homeomorfismos e abertos da definição acima. O par $\left(\mathcal{U}_{\alpha}, \varphi_{\alpha}\right)$ é chamado de carta local e, sendo $p \in \mathcal{U}_{\alpha}$, costumamos dizer que $\mathcal{U}_{\alpha}$ é vizinhança coordenada de $p, \varphi_{\alpha}$ é coordenada local em $p$ e sua inversa $\varphi_{\alpha}^{-1}$ parametrização local em $p$. A composição $\varphi_{\beta}^{-1} \circ \varphi_{\alpha}$ é chamada de mudança de coordenadas, e uma família $\left\{\left(\mathcal{U}_{\alpha}, \varphi_{\alpha}\right)\right\}$ satisfazendo 1) e 2) da definição acima é chamada atlas suave (analítico) de $M$. No caso em que 3) é satisfeita, dizemos que $M$ possui uma estrutura suave (analítica).

Definição 1.3. Sejam $M$ e $N$ duas variedades suaves (analíticas), com respectivas estruturas $\left\{\left(\mathcal{U}_{\alpha}, \psi_{\alpha}\right)\right\}$ e $\left\{\left(\mathcal{V}_{\beta}, \varphi_{\beta}\right)\right\}$. Dizemos que uma aplicação $f: M \longrightarrow N$ é suave (analítica) em $p \in M$ se $\varphi_{\beta} \circ f \circ \psi_{\alpha}^{-1}$ for suave (analítica) em $\psi_{\alpha}(p)$. Vamos denotar o conjunto das funções suaves de $M$ em $N$ por $\mathcal{C}^{\infty}(M, N)$, e $\mathcal{C}^{\infty}(M)$ o conjunto das funções suaves com domínio $M$ e contra-domínio $\mathbb{R}$.

Observação 1.4. Diremos que a função $f$ é suave se ela for suave em todos os pontos de seu domínio. Note ainda que a definição 1.3 independe da escolha das cartas.

Definição 1.5 (Grupo de Lie). Uma variedade suave (analítica) G é chamada Grupo de Lie se:

a) $G$ é grupo;

b) As aplicações

$$
\begin{aligned}
& \cdot: G \times G \longrightarrow G \quad \text { e } \quad i: G \longrightarrow G \\
& (a, b) \longmapsto a \cdot b \quad \text { e } \quad a \longmapsto a^{-1}
\end{aligned}
$$

são suaves (analíticas).

Observação 1.6. Na definição acima, a condição (b) é equivalente a afirmar que

$$
\begin{aligned}
G \times G & \longrightarrow G \\
(a, b) & \longmapsto a \cdot b^{-1}
\end{aligned}
$$

é suave (analítica).

Teorema 1.7. Seja $G$ um grupo de Lie suave. Então, $G$ admite uma estrutura analítica que o torna um grupo de Lie analítico.

Este resultado é enunciado devido à sua relevância, apesar de que neste texto trabalharemos apenas com estruturas suaves. Sua demonstração foge ao escopo deste texto, mas pode ser encontrada em $[\mathrm{DK}]$, pg. 28. Convém observar, porém, que todos os resultados aqui apresentados também são válidos em grupos de Lie analíticos.

Exemplo 1.8. Alguns exemplos de Grupos de Lie são: 
a) $\left(\mathbb{R}^{n},+\right)$;

b) $\left(S^{1}, \cdot\right)$ sendo $\cdot$ a multiplicação complexa dada por $e^{i \theta} \cdot e^{i \sigma}=e^{i(\theta+\sigma)}$

c) $T^{n}=\underbrace{S^{1} \times \ldots \times S^{1}}_{n}$, com a operação obtida a partir da regra usual para o produto cartesiano de $\left(S^{1}, \cdot\right)$;

d) $G L_{n}(\mathbb{R})$, o grupo multiplicativo das matrizes quadradas de ordem $n$ inversíveis sobre $\mathbb{R}$;

e) $S L_{n}(\mathbb{R})$, o grupo das matrizes reais quadradas de ordem $n$, com determinante 1 ;

f) $O_{n}=\left\{A \in G L_{n}(\mathbb{R}): A^{-1}=A^{t}\right\}$;

g) $U_{n}=\left\{A \in G L_{n}(\mathbb{C}): A^{-1}=\bar{A}^{t}\right\}$

Observação 1.9. Cabem alguns comentários com relação aos exemplos d), e), f) e g) apresentados acima. O espaço das matrizes quadradas $M_{n}(\mathbb{R})$ possui uma estrutura natural de variedade, e considerando a aplicação determinante (a qual é suave) obtemos $G L_{n}(\mathbb{R})=\operatorname{det}^{-1}(\mathbb{R}-\{0\})$. Portanto, $G L_{n}(\mathbb{R})$ é um aberto de $M_{n}(\mathbb{R})$ e possui estrutura de variedade. A multiplicação de matrizes é polinomial em cada componente, e a inversa é dada por:

$$
A^{-1}=\frac{1}{\operatorname{det} A} \cdot \operatorname{adj} A
$$

onde adj $A$ denota a adjunta clássica da matriz $A$ dada por $(\operatorname{adj} A)_{i j}=(-1)^{i+j} \operatorname{det} M_{j i}$, sendo $M_{j i}$ obtida pela omissão da j-ésima linha e i-ésima coluna da matriz $A$. Consequentemente, tais aplicações são suaves e, $\operatorname{como} \operatorname{det}(A \cdot B)=\operatorname{det} A \cdot \operatorname{det} B, \operatorname{resulta}$ que $G L_{n}(\mathbb{R})$ é grupo de Lie.

Usando as funções det e $f(X)=X X^{t}$ (esta última também suave por ter entradas polinomiais), temos que $S L_{n}(\mathbb{R})=\operatorname{det}^{-1}(1)$ e $O_{n}=f^{-1}(I)$. Fixando uma matriz $A$, é válido que $\operatorname{det}(A X)=$ $\operatorname{det} A \operatorname{det} X$ e $f(A X)=A f(X) A^{t}$, para toda matriz $X$; disso segue que det e $f(X)=X X^{t}$ são aplicações de posto constante e, por consequência do teorema do posto, $S L_{n}(\mathbb{R})$ e $O_{n}$ são subvariedades (def. 1.10) de $G L_{n}(\mathbb{R})$. A proposição 1.12 vai garantir que essas subvariedades são grupos de Lie. (O caso $U_{n}$ é análogo, usando a função $\left.g(X)=X \bar{X}^{t}\right)$.

Definição 1.10 (Imersão, Mergulho, Subvariedade).

a) Uma aplicação suave entre variedades $f: M \longrightarrow N$ é imersão se $d f_{p}$ é injetora, $\forall p \in M$.

b) Uma imersão $f: M \longrightarrow N$ é mergulho se $f: M \longrightarrow f(M)$ é homeomorfismo, considerando $f(M)$ com a topologia induzida de $N$.

c) Dada uma variedade $M$ e um subconjunto $N \subset M$, diremos que $N$ é subvariedade imersa de $M$ se $N$ possui uma estrutura suave na qual a inclusão $i: N \longrightarrow M$ é uma imersão. Se existir uma estrutura suave de $N$ em que a inclusão seja um mergulho, $N$ será chamada subvariedade (mergulhada) de $M$. 
Lema 1.11. Sejam $L, M$ variedades e $N$ uma subvariedade mergulhada de $M$. Seja $f: L \longrightarrow M$ suave tal que $f(L) \subset N$. Então, $f: L \longrightarrow N$ é suave.

Demonstração: Tomemos um ponto arbitrário $p \in L$, e seja $q=f(p)$ sua imagem. Podemos considerar uma vizinhança coordenada $(\mathcal{U}, \varphi)$ de $p$ a qual é levada por $f$ em uma vizinhança coordenada $(\mathcal{V}, \psi)$ de $q$ tal que $\psi(\mathcal{V})$ é uma bola $\mathcal{B}_{\varepsilon}(0)$ e $\psi(q)=(0, \ldots, 0) \in \mathbb{R}^{n}$. Como $N$ é subvariedade mergulhada de $M$, temos que $\psi(\mathcal{V} \cap N)=\mathcal{W} \cap\left\{\left(x_{1}, \ldots, x_{m}, 0, \ldots, 0\right) \in \mathbb{R}^{n}\right\}$, sendo $\mathcal{W}$ um aberto de $\mathbb{R}^{n}$. Assim, temos que $\psi \circ f \circ \varphi(x)=\left(f_{1}(x), \ldots, f_{m}(x), 0, \ldots, 0\right)$, para todo $x \in$ $\varphi^{-1}(\mathcal{U})$. Tomando $\pi$ a projeção nas $m$ primeiras componentes (a qual é uma aplicação suave), temos $\pi \circ \psi \circ f \circ \varphi(x)=\left(f_{1}(x), \ldots, f_{m}(x)\right)$ uma aplicação suave em $\varphi^{-1}(p)$. Como $p$ foi tomado arbitrário, concluímos que $f: L \longrightarrow N$ é suave.

Proposição 1.12. Seja $G$ um grupo de Lie e $H$ uma subvariedade mergulhada de $G$ a qual é um grupo com relação ao produto de $G$. Então $H$ é também um grupo de Lie.

Demonstração: Considere a aplicação

$$
\begin{aligned}
f: H \times H & \longrightarrow G \\
\left(h_{1}, h_{2}\right) & \longmapsto h_{1} \cdot h_{2}^{-1}
\end{aligned}
$$

a qual é suave pela definição de grupo, e além disso $f(H \times H) \subset H$. Como assumimos $H$ subvariedade mergulhada de $G$, resulta do lema 1.11 que $f: H \times H \longrightarrow H$ é uma função suave, concluindo portanto que $H$ possui estrutura de grupo de Lie.

Definimos a seguir o conceito de álgebra de Lie:

Definição 1.13 (Álgebra de Lie). Uma Álgebra de Lie $\mathfrak{g}$ é um espaço vetorial (real) dotado de uma aplicação bilinear

$$
[,]: \mathfrak{g} \times \mathfrak{g} \longrightarrow \mathfrak{g}
$$

chamada colchete de Lie, satisfazendo às propriedades:

i) $[X, Y]=-[Y, X]$ (anti-comutatividade);

ii) $[[X, Y], Z]+[[Y, Z], X]+[[Z, X], Y]=0$ (equação de Jacobi);

Exemplo 1.14. Alguns exemplos de álgebras de Lie são:

a) $\mathcal{M}_{n}(\mathbb{R})$, com $[A, B]=A B-B A$;

b) $\mathbb{R}^{3}$ com o produto vetorial $[v, w]=v \times w$; 


\subsection{Colchete de Campos}

Definimos recentemente álgebra de Lie, onde a aplicação colchete é essencial. Dada uma variedade, vamos agora pensar no módulo dos campos suaves em uma variedade e estabelecer uma aplicação do tipo colchete. Na verdade, mostraremos que esta aplicação é de fato um colchete de Lie, dando o primeiro exemplo prático desta operação. O conteúdo necessário para trabalharmos em campos em uma variedade será dado nesta seção.

Definição 1.15 (Campo em Variedade). Um campo $X$ em uma variedade $M$ é uma seção do fibrado tangente $T M:=\cup_{p \in M} T_{p} M$ (cf. [dC2], pg. 15), ou seja, é uma aplicação

$$
X: M \longrightarrow T M \quad \text { tal que } \quad \pi \circ X(p)=p
$$

onde $\pi: T M \longrightarrow M$ é a projeção canônica.

Observação 1.16. Tomando um ponto $p \in M$ (m-dimensional) e considerando $x$ uma parametrização local em $p$, podemos escrever:

$$
X(p)=\sum_{i=1}^{m} a_{i}(p) \frac{\partial}{\partial x_{i}}
$$

sendo cada $a_{i}$ uma função real e $\left\{\frac{\partial}{\partial x_{i}}\right\}_{i=1 \ldots n}$ base de $T_{p} M$ associada a $x$.

O campo $X$ será suave em $p$ se para todo $i, a_{i} \circ x$ for suave em $x^{-1}(p)$. Vamos denotar o conjunto dos campos suaves em uma variedade $M$ por $\Xi(M)$.

Definição 1.17 (Derivada Direcional). Seja $v \in T_{p} M$. Definimos a derivada direcional em $p \in M$ na direção de $v$ de uma função $f \in \mathcal{C}^{\infty}(M)$ por:

$$
\begin{aligned}
v \cdot f_{p} & :=d f_{p}(v) \\
& =\left.\frac{d}{d t}(f \circ \alpha)\right|_{t=0}
\end{aligned}
$$

onde $\alpha:(-\varepsilon, \varepsilon) \longrightarrow M, \alpha(0)=p$ e $\alpha^{\prime}(0)=v$.

Observação 1.18. A definição acima nos dá outra interpretação de um campo: podemos pensá-lo como uma aplicação entre espaços de funções. De fato, dada uma função $f \in \mathcal{C}^{\infty}(M)$, definimos $X f(p)=X_{p} f$, ou seja, a derivada direcional de $f$ na direção do campo. Por (1.1), temos que:

$$
X f(p)=\sum_{i=1}^{m} a_{i}(p) \frac{\partial f}{\partial x_{i}}
$$


Expressando desse modo temos que $X$ é suave se, e somente se, $X f$ é suave para todo $f \in \mathcal{C}^{\infty}(M)$.

Como uma variedade suave é localmente difeomorfa a $\mathbb{R}^{n}$, os teoremas locais de equações diferenciais ordinárias são naturalmente estendidos a variedades suaves. Por isso podemos admitir o seguinte resultado, o qual pode ser verificado em qualquer livro de equações diferenciais ordinárias:

Teorema 1.19. Seja $X$ um campo suave em uma variedade $M$ e $p \in M$. Então existe uma vizinhança $\mathcal{U}$ de $p$ em $M$, um intervalo $(-\delta, \delta) \operatorname{com} \delta>0$ e uma aplicação suave $\varphi:(-\delta, \delta) \times \mathcal{U} \longrightarrow$ $M$ tal que a curva $t \longmapsto \varphi(t, q), t \in(-\delta, \delta), q \in \mathcal{U}$ é a única curva que satisfaz $\frac{\partial \varphi}{\partial t}=X(\varphi(t, q)) e$ $\varphi(0, q)=q$.

Definição 1.20. A aplicação $\varphi_{t}: \mathcal{U} \longrightarrow M$ sendo $\varphi_{t}(q)=\varphi(t, q)$ dada pelo teorema 1.19 é chamada fluxo local de $X$.

Definição 1.21 (Colchete de Campos). Sejam $X$ e $Y$ campos em $M$. Definimos o campo $[X, Y]$ por:

$$
[X, Y] f=X(Y f)-Y(X f), \quad \forall f \in \mathcal{C}^{\infty}(M)
$$

Observação 1.22 (Expressão em coordenadas). Em uma variedade $n$-dimensional, considere $X=$ $\sum_{i=1}^{n} a_{i} \frac{\partial}{\partial x_{i}}$ e $Y=\sum_{j=1}^{n} b_{j} \frac{\partial}{\partial x_{j}}$. Assim,

$$
\begin{aligned}
X Y & =X\left(\sum_{j=1}^{n} b_{j} \frac{\partial}{\partial x_{j}}\right) \\
& =\sum_{i=1}^{n} a_{i} \frac{\partial}{\partial x_{i}}\left(\sum_{j=1}^{n} b_{j} \frac{\partial}{\partial x_{j}}\right) \\
& =\sum_{i=1}^{n} \sum_{j=1}^{n} a_{i}\left(\frac{\partial b_{j}}{\partial x_{i}} \frac{\partial}{\partial x_{j}}+b_{j} \frac{\partial^{2}}{\partial x_{i} \partial x_{j}}\right) \\
& =\sum_{i, j=1}^{n} a_{i} \frac{\partial b_{j}}{\partial x_{i}} \frac{\partial}{\partial x_{j}}+\sum_{i, j=1}^{n} a_{i} b_{j} \frac{\partial^{2}}{\partial x_{i} \partial x_{j}}
\end{aligned}
$$

Analogamente,

$$
Y X=\sum_{i, j=1}^{n} b_{j} \frac{\partial a_{i}}{\partial x_{j}} \frac{\partial}{\partial x_{i}}+\sum_{i, j=1}^{n} a_{i} b_{j} \frac{\partial^{2}}{\partial x_{i} \partial x_{j}}
$$

Portanto, 


$$
\begin{aligned}
{[X, Y] } & =X Y-Y X \\
& =\sum_{i, j=1}^{n} a_{i} \frac{\partial b_{j}}{\partial x_{i}} \frac{\partial}{\partial x_{j}}-\sum_{i, j=1}^{n} b_{j} \frac{\partial a_{i}}{\partial x_{j}} \frac{\partial}{\partial x_{i}}
\end{aligned}
$$

resultando:

$$
[X, Y]=\sum_{i, j=1}^{n}\left(a_{i} \frac{\partial b_{j}}{\partial x_{i}}-b_{i} \frac{\partial a_{j}}{\partial x_{i}}\right) \frac{\partial}{\partial x_{j}}
$$

Observação 1.23. Em particular, quando $M$ é aberto de $\mathbb{R}^{n}, X=\left(x_{1}, \ldots, x_{n}\right)$ e $Y=\left(y_{1}, \ldots y_{n}\right)$, temos:

$$
\begin{aligned}
{[X, Y] } & =D_{Y} X-D_{X} Y \\
& =D_{X} Y-D_{Y} X
\end{aligned}
$$

onde $D$ é a derivada de aplicações de $\mathbb{R}^{n}$ em $\mathbb{R}^{n}$.

Observação 1.24. Vamos verificar que o colchete de campos é um colchete de lie:

1.

$$
\begin{aligned}
{[X, Y] } & =X Y-Y X \\
& =-(Y X-X Y) \\
& =-[Y, X]
\end{aligned}
$$

2.

$$
\begin{aligned}
{[[X, Y], Z]+[[Y, Z] X]+[[Z, X], Y] } & =[X Y-Y X, Z]+[Y Z-Z Y, X]+[Z X-X Z, Y] \\
& =0
\end{aligned}
$$

Proposição 1.25 (cf. [CL], pg. 213).

$$
[X, Y]=0 \Leftrightarrow \varphi_{t}^{X} \circ \varphi_{s}^{Y}=\varphi_{s}^{Y} \circ \varphi_{t}^{X}
$$

\subsection{Campos Invariantes à Esquerda}

Dado um grupo de Lie $G$, considere a aplicação: 


$$
\begin{aligned}
L_{g}: G & \longrightarrow G \\
a & \longmapsto g \cdot a
\end{aligned}
$$

Diremos que um campo $X$ é invariante à esquerda se

$$
X_{g \cdot a}=d L_{g} X_{a}
$$

De modo mais simples, denotamos apenas $X=d L_{g} X, \forall g \in G$.

Analogamente, considerando a função:

$$
\begin{aligned}
R_{g}: G & \longrightarrow G \\
a & \longmapsto a \cdot g
\end{aligned}
$$

vamos dizer que o campo $X$ é invariante à direita se $X=d R_{g} X$. O campo será bi-invariante se for invariante à direita e à esquerda.

Lema 1.26. Campos invariantes à esquerda (ou à direita) são suaves.

Demonstração: Seja $g$ pertencente a uma vizinhança da identidade $e$, e considere a função $L(g, h)=g \cdot h$. Temos assim $D L: T G \times T G \longrightarrow T G$ e, definindo

$$
\begin{aligned}
s: G & \longrightarrow T G \times T G \\
g & \longmapsto\left(g, X_{e}\right)
\end{aligned}
$$

segue que $\pi \circ D L \circ s(g)=g$ e $X(g)=D L \circ s(g)$. Isto prova que o campo é suave.

Dado um vetor $v \in T_{e} G$, podemos definir o campo invariante à esquerda $X$ por $X_{g}=d L_{g} v_{e}$. Com isto temos um campo invariante à esquerda em $G$ de maneira tal que $X_{e}=v$.

Proposição 1.27. O conjunto dos campos invariantes à esquerda num grupo de Lie G tem estrutura de álgebra de Lie.

Demonstração: Primeiro, mostremos que tem estrutura de espaço vetorial. A soma e a multiplicação por escalar são induzidas das operações usuais com funções, faltando verificar que os resultados destas operações ainda são invariantes à esquerda. Tomando dois campos invariantes à esquerda $X$ e $Y$, temos que $X=d L_{g} X$ e $Y=d L_{g} Y$. Assim,

$$
\begin{aligned}
X+Y & =d L_{g} X+d L_{g} Y \\
& =d L_{g}(X+Y)
\end{aligned}
$$

Logo, a soma de campos invariantes à esquerda é invariante à esquerda. Agora, tome $\alpha \in \mathbb{R}$, 


$$
\begin{aligned}
\alpha X & =\alpha d L_{g} X \\
& =d L_{g}(\alpha X)
\end{aligned}
$$

ficando clara a estrutura de espaço vetorial. Já provamos na observação 1.24 que o colchete de campos é colchete de Lie, mas precisamos verificar que o colchete de campos invariantes à esquerda é ainda invariante à esquerda:

$$
\begin{aligned}
d L_{g}[X, Y] f & =d L_{g}(X Y-Y X) f \\
& =\left(d L_{g} X\right) Y f-\left(d L_{g} Y\right) X f \\
& =X Y f-Y X f \\
& =[X, Y] f
\end{aligned}
$$

Concluímos que o conjunto dos campos invariantes à esquerda em um dado grupo de Lie é uma álgebra de Lie.

Definição 1.28. A álgebra de Lie dos campos invariantes à esquerda sobre um grupo de lie $G$ é chamada álgebra de Lie do grupo de Lie, e denotada por $\mathfrak{g}$.

Observação 1.29 .

$$
\mathfrak{g} \cong T_{e} G
$$

Lembre-se que a cada vetor de $T_{e} G$, está associado um único campo invariante à esquerda. Do mesmo modo, dado um campo $X$, ele nos dá um único vetor $X_{e}$, evidenciando a biunivocidade entre $\mathfrak{g}$ e $T_{e} G$. Em $T_{e} G$, definimos

$$
\left[X_{e}, Y_{e}\right]:=[X, Y]
$$

onde $X$ e $Y$ são os campos invariantes à esqueda construídos a partir de $X_{e}, Y_{e} \in T_{e} G$ (veja comentário no início desta seção). Isto dá a $T_{e} G$ uma estrutura de álgebra de Lie que, por construção, é isomorfa a $\mathfrak{g}$

Finalizamos esta seção apresentando o terceiro teorema fundamental de Lie, o qual será usado em diversas situações ao longo do texto. Omitimos sua demonstração por estar além dos nossos objetivos, mas pode ser conferida em [DK], pg. 32 .

Teorema 1.30 (30 Teorema de Lie). Seja $\mathfrak{g}$ uma álgebra de Lie de dimensão finita. Então, existe um único grupo de Lie simplesmente conexo $G$ que tem álgebra de Lie $G$. 


\subsection{Subgrupos e Homomorfismos de Lie}

Nesta seção, estamos preocupados em estabelecer relações entre os subgrupos de Lie e as subálgebras de Lie. Sob que condições um subgrupo de Lie de um grupo de Lie $G$ possui uma álgebra de Lie que coincide com uma sub-álgebra de Lie de $\mathfrak{g}$ ? Será possível criar um homomorfismo de álgebras de Lie a partir de um homomorfismo de grupos de Lie? E a recíproca, será possível? Em que condições?

Estas perguntas norteiam esta seção, o que a torna repleta de resultados importantes da teoria dos grupos de Lie. Alguns conceitos necessários serão rapidamente introduzidos, como o de folheação, distribuição e recobrimento mas, para evitar o desvio de nosso objetivo principal, usaremos alguns resultados sempre dando indicações de onde podem ser encontrados.

Definição 1.31 (Homomorfismo de Grupos de Lie). Sejam $G$ e $H$ grupos de Lie. Uma aplicação $\varphi: G \longrightarrow H$ é homomorfismo de grupos de Lie se:

a) $\varphi$ é suave.

b) $\varphi\left(g_{1} \cdot g_{2}\right)=\varphi\left(g_{1}\right) \cdot \varphi\left(g_{2}\right)$

Definição 1.32 (Homomorfismo de Álgebras de Lie). Dadas duas álgebras de Lie $\mathfrak{g}$ e $\mathfrak{h}$, a aplicação $\psi: \mathfrak{g} \longrightarrow \mathfrak{h}$ é homomorfismo de álgebras de Lie se $\psi$ é linear (no sentido de espaço vetorial) e, além disso, $\psi[X, Y]=[\psi X, \psi Y]$.

Em algumas demonstrações faremos uso do teorema de Frobenius, o qual enunciamos em duas versões. A seguir colocamos rapidamente os conceitos envolvidos, mas para um estudo mais cuidadoso recomendamos o apêndice dois de [CL] (vide pg. 215).

Definição 1.33 (Distribuição). Uma distribuiçãa $D$ de dimensão $k$ em uma variedade $M^{m}$ é uma escolha de $k$-subespaços vetoriais em $T M$, isto é,

$$
D_{p} \subset T_{p} M, \quad \forall p \in M .
$$

Diremos distribuição suave se, para cada $p \in M$, existir uma vizinhança $\mathcal{V}$ de $p$ e campos suaves $X_{1}, \ldots, X_{k}$ definidos em $\mathcal{V}$ satisfazendo, para todo $q \in \mathcal{V}:$

a) $X_{i}(q) \in D_{q}, \forall i=1, \ldots, k$

b) $\left\{X_{i}(q)\right\}_{1 \leq i \leq k}$ é base de $D_{q}$.

E, ainda, a distribuição será involutiva se satisfizer a seguinte condição:

"Sejam $X, Y$ campos definidos em uma vizinhança $\mathcal{U}$ de $p$ em $M$, tais que $X_{q}, Y_{q} \in D_{q}, \forall q \in \mathcal{U}$. Então $[X, Y]_{q} \in D_{q}, \forall q \in \mathcal{U}$ ".

Teorema 1.34 (Frobenius). Seja D uma k-distribuição suave involutiva em uma variedade $M$. Então: 
a) Dado $p \in M$, existe uma subvariedade $L_{p}$ tal que

$$
T_{x} L_{p}=D_{x}, \quad \forall x \in L_{p}
$$

A subvariedade acima é chamada subvariedade integral

b) Se $L^{1}$ e $L^{2}$ são subvariedades integrais máximas e $T_{p} L^{1}=T_{p} L^{2}$, então $L^{1}=L^{2}$.

c) (quase mergulhada) Supondo $K$ outra variedade e $\psi: K \longrightarrow L$ uma função tal que $i \circ \psi: K \longrightarrow$ $M$ é suave, a função $\psi: K \longrightarrow L$ também é suave.

Definição 1.35 (Folheação). Uma folheação de dimensão $k$ de uma variedade $M^{m}$ é uma partição de $M$ por uma família de subvariedades $\mathcal{F}$ (todas de dimensão $k$ ) de maneira que, para cada $p \in M$ e cada $v \in T_{p} F_{p}$ (sendo $F_{p}$ a folha que contém $p$ ) existe um campo suave $X$ definido numa vizinhança $\mathcal{U}$ de $p$ em $M$ satisfazendo:

a) $X_{p}=v$

b) $X_{q} \in T_{q} F_{q}, \quad \forall q \in \mathcal{U}$

Observação 1.36. Se $\mathcal{F}=\{F\}$ é uma folheação de dimensão $k$ de uma variedade $M^{m}$, então para todo $p \in M$, existe uma vizinhança $\mathcal{U}$ de $p$, uma vizinhança $\mathcal{W}$ de $0 \in \mathbb{R}^{m}=\mathbb{R}^{k} \times \mathbb{R}^{m-k}$ e um difeomorfismo

$$
\psi: \mathcal{W} \longrightarrow \mathcal{U}
$$

tal que $\psi\left(\mathcal{W} \cap\left(\mathbb{R}^{k} \times\left\{y_{0}\right\}\right)=\mathcal{P}_{\psi\left(0, y_{0}\right)}\right.$, onde $\mathcal{P}_{\psi\left(0, y_{0}\right)}$ é a componente conexa de $F_{\psi\left(0, y_{0}\right)} \cap \mathcal{U}$ que contém $\psi\left(0, y_{0}\right)$.

Teorema 1.37 (Frobenius Reformulado). Seja $D$ uma $k$-distribuição suave involutiva em uma variedade $M$. Então, existe uma única folheação $\mathcal{F}=\{F\}$ de dimensão $k$ satisfazendo $D_{q}=$ $T_{q} F_{q}, \quad \forall q \in M$.

Definição 1.38 (Subgrupo de Lie). Seja $G$ um grupo de Lie. Dizemos que $H \subset G$ é subgrupo de Lie de $G$ se:

a) $H$ é subgrupo algébrico de $G$.

b) $H$ é subvariedade imersa de $G$.

c)

$$
\begin{aligned}
H \times H & \longrightarrow H \\
(a, b) & \longmapsto a \cdot b^{-1}
\end{aligned}
$$

é suave. 
Definição 1.39 (Campos $f$-relacionados). Dada uma função suave entre variedades $f: M \longrightarrow N$, $X \in \Xi(M), Y \in \Xi(N)$. Dizemos que $X$ é $f$-relacionado a $Y$ se

$$
Y_{f(p)}=d f_{p} X_{p}, \forall p \in M
$$

Observação 1.40. Outra maneira de trabalhar com campos $f$-relacionados é tomando uma função $g \in \mathcal{C}^{\infty}(N)$ e, conforme a observação 1.18 , fazer:

$$
\begin{aligned}
Y_{f(p)}(g) & =d f_{p} X_{p}(g) \\
& =X_{p}(g \circ f)
\end{aligned}
$$

daí, $X$ e $Y$ são $f$-relacionados se, e somente se, para toda função $g \in \mathcal{C}^{\infty}(N)$, ocorrer $(Y g) \circ f=$ $X(g \circ f)$.

Proposição 1.41. Sejam $X^{1}, X^{2} \in \Xi(M)$ e $Y^{1}, Y^{2} \in \Xi(N)$. Suponha $X^{i}$ f-relacionado a $Y^{i}$, $i=1,2$. Então, $\left[X^{1}, X^{2}\right]$ é f-relacionado a $\left[Y^{1}, Y^{2}\right]$.

Demonstração: Tome $g \in \mathcal{C}^{\infty}(N)$. Segundo a observação 1.40, temos:

$$
\left(Y^{i} g\right) \circ f=X^{i}(g \circ f), \quad i=1,2
$$

Usando a eq. 1.4, temos:

$$
\begin{aligned}
\left(\left[Y^{1}, Y^{2}\right] g\right) \circ f & =\left(Y^{1}\left(Y^{2} g\right)\right) \circ f-\left(Y^{2}\left(Y^{1} g\right)\right) \circ f \\
& =X^{1}\left(\left(Y^{2} g\right) \circ f\right)-X^{2}\left(\left(Y^{1} g\right) \circ f\right) \\
& =X^{1}\left(X^{2}(g \circ f)\right)-X^{2}\left(X^{1}(g \circ f)\right) \\
& =\left[X^{1}, X^{2}\right](g \circ f)
\end{aligned}
$$

Definição 1.42 (Subálgebra de Lie). Seja $\mathfrak{g}$ uma álgebra de Lie. Um subespaço h é uma subálgebra de Lie se é fechado com relação à operação colchete.

Lema 1.43. Seja $H \subset G$ um subgrupo de Lie. Então hy é subálgebra de Lie de $\mathfrak{g}$.

Demonstração: Sendo $a \in H$, considere a aplicação $L_{a}^{H}:=\left.L_{a}\right|_{H}$. Temos assim que $i \circ L_{a}^{H}=L_{a} \circ i$, sendo $i: H \longrightarrow G$ a inclusão. Seja $X \in T_{e} H$, e defina os campos $X_{a}^{H}=d L_{a}^{H} X, \forall a \in H$ e $X_{a}=d L_{a} X, \forall a \in G$. Mostremos que $X$ e $X^{H}$ são $i$-relacionados: 


$$
\begin{aligned}
X_{i(a)} & =d L_{i(a)} d i X \\
& =d i d L_{a}^{H} X \\
& =d i_{a} X^{H}
\end{aligned}
$$

Como consequência da proposição 1.41 , resulta que $[X, Y]_{i(e)}=d i_{e}\left[X^{H}, Y^{H}\right] \in d i\left(T_{e} H\right) \cong T_{e} H$.

Teorema 1.44. Seja $G$ um grupo de Lie com álgebra de Lie $\mathfrak{g}$. Seja $\mathfrak{h}$ subálgebra de Lie de $\mathfrak{g}$. Então existe um único subgrupo de Lie conexo $H \subset G$ com álgebra de Lie $\mathfrak{h}$.

Demonstração: Defina $D_{q}=\left\{X_{q}: X_{q}=d L_{q} X\right.$, com $\left.X \in \mathfrak{h}\right\}$. Como h é uma álgebra de Lie, então $D_{q}$ é involutiva. Pelo teorema de Frobenius (teorema 1.37), existe uma única folheação $\mathcal{F}$ tal que $D_{q}=T_{q} F_{q}, \forall q$. Definindo $H=F_{e}$, temos que:

i) $L_{g}$ leva folha em folha, pois:

$$
\begin{aligned}
d L_{g} D_{a} & =d L_{g} T_{a} F_{a} \\
& =T_{g \cdot a} F_{g \cdot a} \\
& =D_{g \cdot a}
\end{aligned}
$$

e portanto $d L_{g} D_{a}=D_{g \cdot a}$.

ii) Se $h \in H$, então $L_{h^{-1}}(H)$ é folha contendo $e$. Portanto $L_{h^{-1}}(H)=H$, resultando que $H$ é grupo.

iii) Defina

$$
\begin{gathered}
\psi: H \times H \longrightarrow H \\
(a, b) \longmapsto a^{-1} \cdot b
\end{gathered}
$$

como $i$ e $i \circ \psi$ são suaves, resulta do teorema de Frobenius (teo. 1.34) que $\psi$ é suave. Assim, $H$ é grupo de Lie conexo com álgebra de Lie $\mathfrak{h}$.

Proposição 1.45. Sejam $G$ e $H$ grupos de Lie com respectivas álgebras de Lie $\mathfrak{g}$ e h. Se $\varphi: G \longrightarrow$ $H$ é homomorfismo de grupos de Lie, então $d \varphi_{e}: \mathfrak{g} \longrightarrow \mathfrak{h}$ é homomorfismo de álgebras de Lie.

Demonstração: Tomando $a \in G$, temos que 


$$
\begin{aligned}
\varphi \circ L_{a}^{G}(x) & =\varphi(a \cdot x) \\
& =\varphi(a) \cdot \varphi(x) \\
& =L_{\varphi(a)}^{H} \circ \varphi(x)
\end{aligned}
$$

Disso, segue que:

$$
d \varphi \circ d L_{a}^{G}=d L_{\varphi(a)}^{H} \circ d \varphi
$$

Sendo $h \in H$ e $g \in G$, vamos definir os campos:

$$
\begin{gathered}
X_{h}^{H}=d L_{h}^{H} d \varphi_{e} X_{e} \\
X_{g}^{G}=d L_{g}^{G} X_{e}
\end{gathered}
$$

Com isso, temos:

$$
\begin{aligned}
X_{\varphi(g)}^{H} & =d L_{\varphi(g)}^{H} d \varphi_{e} X_{e} \\
& =d \varphi d L_{g}^{G} X_{e} \\
& =d \varphi X_{g}^{G}
\end{aligned}
$$

E portanto os campos $X^{H}$ e $X^{G}$ são $\varphi$-relacionados. Resulta da prop.1.41 que:

$$
\left[X^{H}, Y^{H}\right]_{e}=d \varphi_{e}[X, Y] \subset d \varphi_{e} T_{e} G
$$

De onde concluimos que $d \varphi_{e}$ é homomorfismo de álgebras de Lie.

Definição 1.46 (Recobrimento). Uma aplicação sobrejetora e contínua $\pi: E \longrightarrow B$ é chamada recobrimento se, para cada $p \in B$, existe vizinhança $\mathcal{U}$ de $p$ tal que $\pi^{-1}(\mathcal{U})$ é a união disjunta de abertos $\left.\mathcal{U}_{\alpha} \subset E \operatorname{com} \pi\right|_{\mathcal{U}_{\alpha}}: \mathcal{U}_{\alpha} \longrightarrow \mathcal{U}$ homeomorfismo.

O seguinte teorema pode ser verificado em $[\mathrm{B}]$, pg. 98, ou então em [DK], pg. 67. As duas 
demonstrações são bem distintas, sendo que a primeira envolve ações (conceito a ser trabalhado no próximo capítulo) e a segunda, homotopia.

Teorema 1.47. Dado um grupo de Lie conexo $G$, existe um único grupo de Lie simplesmente conexo $\widetilde{G}$ com homomorfismo de Lie $\pi: \widetilde{G} \longrightarrow$ G que é também recobrimento.

Proposição 1.48. Sejam $G, H$ grupos de Lie conexos e $\pi: G \longrightarrow H$ homomorfismo de Lie sobrejetor.

$$
\pi \quad \text { é recobrimento } \Leftrightarrow \quad d \pi_{e} \quad \text { é isomorfismo. }
$$

\section{Demonstração:}

$(\Leftarrow)$ Suponha $d \pi_{e}$ isomorfismo, $d \pi_{e}: T_{e} G \longrightarrow T_{e^{\prime}} H$, onde $e^{\prime}$ denota o elemento neutro de $H$.

Do teorema da função inversa, existe vizinhança $\mathcal{U}$ de $e$ e vizinhança $\mathcal{W}$ de $e^{\prime}$ tal que $\pi: \mathcal{U} \longrightarrow \mathcal{W}$ é difeomorfismo. Como $\pi$ é sobrejetor, dado $p \in H$ é possível encontrar $q \in G$ tal que $\pi(q)=p$. Daí, $\pi: q \cdot \mathcal{U} \longrightarrow p \cdot \mathcal{W}$ é difeomorfismo, pois $\pi(q \cdot x)=\pi(q) \cdot \pi(x)=p \cdot \pi(x)$. Logo $\pi$ é recobrimento.

$(\Rightarrow)$ Como $\pi$ é recobrimento, existe uma vizinhança $\mathcal{V}$ de $e$ tal que $\pi: \mathcal{V} \longrightarrow \mathcal{U}$ é homeomorfismo. Além disso, como por hipótese $\pi$ é homomorfismo de Lie, segue conjuntamente com a proposição 1.45 que $d \pi_{e}$ é isomorfismo de álgebras de Lie.

Teorema 1.49. Sejam $G, H$ grupos de Lie e $\theta: \mathfrak{g} \longrightarrow \mathfrak{h}$ homomorfismo de algebras de Lie. Então:

1. Existe uma vizinhança $\mathcal{U}$ de e e aplicação $\varphi: \mathcal{U} \longrightarrow H$ suave tal que

(a) $\varphi(a b)=\varphi(a) \varphi(b)$ para $a, b, a b \in \mathcal{U}$ (homomorfismo local).

(b) $d \varphi_{e}=\theta$.

2. Se $G$ for simplesmente conexo, então existe um homomorfismo $\varphi: G \longrightarrow H$ tal que $d \varphi_{e}=\theta$.

3. Se $G$ é conexo e existem dois homomorfismos $\varphi, \psi: G \longrightarrow H$ com $d \varphi_{e}=d \psi_{e}=\theta$ então $\varphi=\psi$.

4. Se $G$ e $H$ são conexos e simplesmente conexos e $\theta$ é um isomorfismo, então $G$ e $H$ são isomorfos.

\section{Demonstração:}

1. Considere $\mathcal{K}=\{(x, \theta(x)): x \in \mathfrak{g}\}$, o qual é subálgebra de Lie de $\mathfrak{g} \times \mathfrak{h}$. Segue do teo.1.44 que existe um subgrupo de Lie conexo $R$ de $G \times H$ com álgebra de Lie $\mathcal{K}$.

Considere a inclusão $i: R \longrightarrow G \times H$ e a projeção $\pi_{1}: G \times H \longrightarrow G$. Então, $\pi_{1} \circ i: R \longrightarrow G$ é suave e um homomorfismo de Lie tal que $d\left(\pi_{1} \circ i\right)(x, \theta(x))=x, \forall x \in \mathfrak{g}$. Segue do Teorema da Função Inversa que existem vizinhanças $\mathcal{U}$ de $e^{\prime}$ em $R$ e $\mathcal{V}$ de $e$ em $G$ tais que $\pi_{1} \circ i: \mathcal{U} \longrightarrow \mathcal{V}$ é difeomorfismo. Vamos definir $\varphi=\pi_{2} \circ\left(\pi_{1} \circ i\right)^{-1}: \mathcal{V} \longrightarrow H$. Logo, $\varphi$ é homomorfismo local e $d \varphi_{e}=\theta$, pois: 


$$
\begin{aligned}
d \varphi_{e}(X) & =d\left(\pi_{2} \circ\left(\pi_{1} \circ i\right)^{-1}\right)_{e}(X) \\
& =d \pi_{2} d\left(\left(\pi_{1} \circ i\right)^{-1}\right) X \\
& =d \pi_{2}(X, \theta(X)) \\
& =\theta(X)
\end{aligned}
$$

2. Conforme fizemos no item 1 desta prova, verificamos que $\pi_{1} \circ i$ é homomorfismo sobrejetor e $d\left(\pi_{1} \circ i\right)_{e}$ é isomorfismo. Segue da prop.1.48 que $\pi_{1} \circ i: R \longrightarrow G$ é recobrimento. Supondo $G$ simplesmente conexo, temos que $\pi_{1} \circ i$ é homeomorfismo e $\varphi=\pi_{2} \circ\left(\pi_{1} \circ i\right)^{-1}: G \longrightarrow H$ é o homomorfismo procurado.

3. Tome $\tilde{\mathcal{K}}=\{\tilde{\theta}, \psi(\tilde{\theta})\}$. $R$ e $\tilde{R}$ são subgrupos de Lie conexos com a mesma álgebra de Lie. Logo $R=\tilde{R}$ e $\varphi=\psi$.

4. Do item (2) desta prova, temos que $\varphi: G \longrightarrow H$ é recobrimento. Como $H$ é conexo, simplesmente conexo, segue que $\varphi$ é isomorfismo.

Proposição 1.50. Seja $G$ um grupo de Lie e $G^{0}$ a componente conexa da identidade. Então:

1. $G^{0}$ é um subgrupo de Lie de $G$ normal.

2. As componentes conexas de $G$ são da forma $g G^{0}$, com $g \in G^{0}$.

3. Dado uma vizinhança $\mathcal{U}$ de e então $G^{0}=\cup_{n=1}^{\infty} \mathcal{U}^{n}$ onde $\mathcal{U}^{n}=\left\{g_{1}^{ \pm} \cdots g_{n}^{ \pm} \mid g_{i} \in \mathcal{U}\right\}$

Demonstração: É verdade que $G^{0}$ é aberto e fechado. Mostremos que é um subgrupo de Lie.

Tome $g \in G^{0}$ arbitrário, e considere o conjunto $g \cdot G^{0}=L_{g}\left(G^{0}\right)$. Como $L_{g}$ é suave, $g \cdot G^{0}$ é conexa e $g \in g \cdot G^{0}$. Isto implica que $g \cdot G^{0} \cap G^{0} \neq \emptyset$ e portanto $g \cdot G_{0}=G_{0}$. Da mesma forma, como a inversão é suave, o conjunto $G_{-1}^{0}=\left\{g^{-1}: g \in G^{0}\right\}$ é conexo com $e \in G_{-1}^{0}$. Disso resulta $G_{-1}^{0}=G^{0}$ e portanto $G^{0}$ é subgrupo de Lie de $G$.

Para ver que $G^{0}$ é normal, basta tomar a função suave $x \longmapsto g \cdot x \cdot g^{-1}$ e temos $g \cdot G^{0} \cdot g^{-1}=G^{0}$, para todo $g \in G$, haja visto que $e \in g \cdot G^{0} \cdot g^{-1}$.

Por fim, basta mostrar que $\cup_{n=1}^{\infty} \mathcal{U}^{n}$ é aberto e fechado em $G^{0}$. Que é aberto não há dúvida, pois $\mathcal{U}$ é aberto e por consequência $\mathcal{U}^{n}$ é aberto, para todo $n$. Verifiquemos que o conjunto é fechado.

Seja $p$ um ponto de acumulação de $\cup_{n=1}^{\infty} \mathcal{U}^{n}$ e $\mathcal{U}^{-1}=\left\{u^{-1}: u \in \mathcal{U}\right\}$. Então, em $p \cdot \mathcal{U}^{-1}$ existe um ponto $g \in \cup_{n=1}^{\infty} \mathcal{U}^{n}$, digamos, $g=p \cdot u^{-1}$, com $u \in \mathcal{U}$. Logo, $p=g \cdot u \in \cup_{n=1}^{\infty} \mathcal{U}^{n}$. Isto conclui a prova. 


\subsection{Aplicação Exponencial e Representação Adjunta}

Definição 1.51. Um homomorfismo de Lie $\varphi: \mathbb{R} \longrightarrow G$ é chamado subgrupo a 1-parâmetro.

Fixando $X \in \mathfrak{g}$, considere o homomorfismo de álgebras de Lie

$$
\begin{aligned}
\theta: \mathbb{R} & \longrightarrow \mathbb{R} \cdot X \\
t & \longmapsto t \cdot X
\end{aligned}
$$

Do teorema 1.49 , existe um único subgrupo a 1-parâmetro $\lambda_{X}: \mathbb{R} \longrightarrow G$ tal que $\lambda_{X}^{\prime}(0)=X$. Com base nisso, definimos:

Definição 1.52. Em um grupo de Lie $G$, a aplicação exponencial de Lie é definida por:

$$
\begin{aligned}
\exp : \mathfrak{g} & \longrightarrow G \\
X & \longmapsto \lambda_{X}(1)
\end{aligned}
$$

Proposição 1.53. A aplicação exponencial de Lie satisfaz às seguintes propriedades:

a) $\exp (t X)=\lambda_{X}(t)$

b) $\exp (-t X)=\exp (t X)^{-1}$

c) $\exp \left(t_{1} X+t_{2} X\right)=\exp \left(t_{1} X\right) \cdot \exp \left(t_{2} X\right)$

d) $\exp : T_{e} G \longrightarrow G$ é suave e $d \exp _{0}=I$

Demonstração: Para mostrar o item a), basta verificar que $\lambda_{X}(t)=\lambda_{t X}(1)$. De fato, considere o grupo a 1-parâmetro $\lambda(s)=\lambda_{X}(s t)$. Assim,

$$
\begin{aligned}
\lambda^{\prime}(0) & =\left.\frac{d}{d t} \lambda_{X}(s t)\right|_{s=0} \\
& =\lambda_{X}^{\prime}(t) \\
& =t X
\end{aligned}
$$

Portanto:

$$
\lambda_{X}(s t)=\lambda_{t X}(s)
$$

e, tomando $s=1$, segue que

$$
\lambda_{X}(t)=\exp (t X)
$$

Os itens b) e c) seguem diretamente do fato de $\exp (t X)$ ser um homomorfismo de Lie e, para verificar d), basta derivar a equação $1.6 \mathrm{em} t=0$. 
Corolário 1.54. Existe uma vizinhança da identidade onde a exponencial de Lie é um difeomorfismo.

Demonstração: Isto segue diretamente do item d) da proposição 1.53 acima e do teorema da função inversa.

Observação 1.55. Alguns fatos interessantes com relação à exponencial de Lie são:

1. Em um grupo de matrizes, a exponencial de Lie coincide com a exponencial de matrizes (cf. [S], pg. 10-21), ou seja,

$$
\exp (A)=\sum_{k=1}^{\infty} \frac{A^{k}}{k !}
$$

2. Em geral, $\exp (X+Y) \neq \exp (X) \cdot \exp (Y)$. (cf. [S], pg 10-55)

3. exp nem sempre é sobrejetora. (cf. [DK], pg. 22)

Proposição 1.56. Seja $\varphi: G \longrightarrow H$ um homomorfismo entre os grupos de Lie $G$ e H. Então $\varphi \circ \exp ^{G}=\exp ^{H} \circ d \varphi$.

Demonstração: $\quad \alpha(t)=\varphi \circ \exp ^{G}(t X)$ e $\beta(t)=\exp ^{H} \circ d \varphi(t X)$ são ambos subgrupo a 1-parâmetro com $\alpha^{\prime}(0)=\beta^{\prime}(0)=d \varphi_{X}$. Segue do teorema 1.49 que $\alpha=\beta$.

A seguir, enunciamos as fórmulas de Campbell. Omitimos sua demonstração, mas pode ser conferida em [S], pg. 10-28.

Teorema 1.57 (Fórmulas de Campbell). Seja $G$ um grupo de Lie e $X, Y \in \mathfrak{g}$. Então, existe $\varepsilon>0$ tal que, para $|t| \leq \varepsilon$, temos:

a) $\exp (t X) \exp (t Y)=\exp \left(t(X+Y)+\frac{t^{2}}{2}[X, Y]+O\left(t^{3}\right)\right)$

b) $\exp (t X) \exp (t Y) \exp (-t X)=\exp \left(t Y+t^{2}[X, Y]+O\left(t^{3}\right)\right)$

c) $\exp (-t X) \exp (-t Y) \exp (t X) \exp (t Y)=\exp \left(t^{2}[X, Y]+O\left(t^{3}\right)\right)$

onde $\frac{O\left(t^{3}\right)}{|t|^{3}}$ é limitado.

Definição 1.58 (Conjugação). Fixado um elemento $g$ em um grupo de Lie $G$, definimos conjugação por $g$ como a aplicação:

$$
\begin{aligned}
C_{g}: G & \longrightarrow G \\
a & \longmapsto g a g^{-1}
\end{aligned}
$$

ou seja, $C_{g}=L_{g} \circ R_{g^{-1}}$. 
Definição 1.59 (Adjunta). A Representação Adjunta de um grupo de Lie $G$ é a função:

$$
\text { Ad } \begin{aligned}
: G & \longrightarrow A u t(\mathfrak{g}) \\
g & \longmapsto d C_{g}
\end{aligned}
$$

ou seja, Ad $(g)=d L_{g} \circ d R_{g^{-1}}$

Observação 1.60. Como consequência da definição, temos que:

$$
A d(g) X=\left.\frac{d}{d t}\left(g \exp (t X) g^{-1}\right)\right|_{t=0}
$$

e, da proposição 1.56 :

$$
g \exp (X) g^{-1}=\exp (\operatorname{Ad}(g) X)
$$

Definição 1.61 (Adjunta pequena). Definimos a adjunta pequena de um grupo de Lie $G$ como a aplicação:

$$
\begin{aligned}
\text { ad } & : \mathfrak{g} \longrightarrow \operatorname{End}(\mathfrak{g}) \\
X & \longmapsto d \operatorname{Ad}_{e} X
\end{aligned}
$$

isto é,

$$
\operatorname{ad}(X) Y=\left.\frac{d}{d t} \operatorname{Ad}(\exp (t X)) Y\right|_{t=0}
$$

Observação 1.62. Usando novamente a proposição 1.56, temos:

$$
\operatorname{Ad}(\exp (X))=\exp (\operatorname{ad}(X))
$$

\section{Lema 1.63.}

$$
\operatorname{ad}(X) Y=[X, Y]
$$

Demonstração: Pelas fórmulas de Campbell (teorema 1.57), temos que:

$$
\exp (t X) \exp (t Y) \exp (-t X)=\exp \left(t Y+t^{2}[X, Y]+O\left(t^{3}\right)\right)
$$

e, pela equação 1.9 , resulta:

$$
\exp (\operatorname{Ad}(\exp (t X)) t Y)=\exp \left(t Y+t^{2}[X, Y]+O\left(t^{3}\right)\right)
$$

Tomando $t$ suficientemente pequeno, vale:

$$
\operatorname{Ad}(\exp (t X)) Y=Y+t[X, Y]+O\left(t^{2}\right)
$$


por fim, derivando, concluimos por meio da equação 1.10 que:

$$
\operatorname{ad}(X) Y=[X, Y]
$$

Proposição 1.64. Seja $G$ um grupo de Lie conexo com álgebra de Lie $\mathfrak{g}$. Então, dados $X, Y \in \mathfrak{g}$, temos que $[X, Y]=0$ se, e somente se, $\exp (X) \exp (Y)=\exp (Y) \exp (X)$. Em particular, se o colchete de Lie for nulo para quaisquer $X, Y \in \mathfrak{g}$, o grupo $G$ será abeliano.

\section{Demonstração:}

$(\Rightarrow)$ Pela observação 1.62, temos que:

$$
\begin{aligned}
\operatorname{Ad}(\exp (X)) Y & =\exp (\operatorname{ad}(X)) Y \\
& =\sum_{k=1}^{\infty} \frac{\operatorname{ad}(X)^{k}}{k !} Y \\
& =Y
\end{aligned}
$$

Pois ad $(X) Y=[X, Y]=0$ (lema 1.63). Usando a equação 1.9, resulta:

$$
\exp (X) \exp (Y) \exp (-X)=\exp (Y)
$$

$(\Leftarrow)$ Supondo

$$
\exp (s X) \exp (t Y) \exp (-s X)=\exp (t Y)
$$

temos, da equação 1.9, que:

$$
\exp (\operatorname{Ad}(\exp (s X)) t Y)=\exp (t Y)
$$

Derivando com relação a $s$ em $s=0$, resulta $[X, Y]=0$. Por meio da proposição 1.50 , segue o resultado para todo o grupo $G$.

\subsection{Subgrupos Fechados}

Teorema 1.65. Seja $G$ um grupo de Lie e $H \subset G$ um subgrupo algébrico fechado de $G$. Então, $H$ é subgrupo de Lie mergulhado. 
Demonstração: Vamos, primeiramente, definir nosso candidato a $T_{e} H$ :

$$
\mathfrak{h}:=\left\{X \in T_{e} G: \exp (t X) \in H, \forall t\right\}
$$

Vamos demonstrar o teorema a partir de uma sequência de afirmações; a primeira delas vai garantir, em particular, que $\mathfrak{h} \neq \emptyset$

Afirmação 1.66. Seja $\left(X_{i}\right)$ uma sequência em $T_{e} G \operatorname{com} X_{i} \longrightarrow X$ e $\left(t_{i}\right)$ sequência real com $t_{i} \longrightarrow 0$. Se $\exp \left(t_{i} X_{i}\right) \in H, \forall i$, então $X \in \mathfrak{h}$.

Note primeiramente que as hipóteses da afirmação 1.66 são satisfeitas em nosso problema, haja visto que podemos supor $H$ não-discreto,e então existe uma sequência $\left(h_{i}\right)$ em $H \operatorname{com} h_{i} \longrightarrow e$. Com isto, podemos tomar $\tilde{X}_{i}$ tal que $\exp \left(\tilde{X}_{i}\right)=h_{i}$, e definir $t_{i}=\left\|\tilde{X}_{i}\right\|, X_{i}=\frac{\tilde{X}_{i}}{t_{i}}$. Como $\left(X_{i}\right)$ é uma sequência na esfera unitária, admite subsequência convergente e portanto as hipóteses estão satisfeitas.

Vamos demonstrar a afirmação 1.66:

Prova: Supondo $t_{i}>0$, definimos

$$
R_{i}(t):=\left[\frac{t}{t_{i}}\right]
$$

ou seja, a função que toma o maior inteiro menor ou igual a $\frac{t}{t_{i}}$. Como:

$$
\frac{t}{t_{i}}-1<R_{i}(t) \leq \frac{t}{t_{i}} \Rightarrow t-t_{i}<t_{i} R_{i}(t) \leq t
$$

concluimos que:

$$
t_{i} R_{i}(t) \longrightarrow t \Rightarrow t_{i} R_{i}(t) X_{i} \longrightarrow t X
$$

Da continuidade da exponencial, resulta:

$$
\exp \left(t_{i} R_{i}(t) X_{i}\right) \longrightarrow \exp (t X)
$$

Por outro lado,

$$
\exp \left(t_{i} R_{i}(t) X_{i}\right)=\exp \left(t_{i} X_{i}\right) \in H
$$

Como $H$ é fechado, $\exp (t X) \in H$ e então $X \in \mathfrak{h}$.

Afirmação 1.67. $\mathfrak{h}$ é um espaço vetorial. 
Prova: Sejam $X, Y \in \mathfrak{h}$, pelas fórmulas de Campbell (teorema 1.57):

$$
\exp \left(t_{i}(X+Y)+\frac{t_{i}^{2}}{2}[X, Y]+O\left(t_{i}^{3}\right)\right)=\exp \left(t_{i} X\right) \exp \left(t_{i} Y\right) \in H
$$

portanto,

$$
\exp \left(t_{i}\left(X+Y+\frac{t_{i}}{2}[X, Y]+O\left(t_{i}^{2}\right)\right)\right) \in H
$$

e

$$
X+Y+\frac{t_{i}}{2}[X, Y]+O\left(t_{i}^{2}\right) \longrightarrow X+Y
$$

quando $t_{i} \longrightarrow 0$. Como consequência da afirmação 1.66 , temos que $X+Y \in \mathfrak{h}$.

Afirmação 1.68. Seja $\mathfrak{h}^{\prime}$ espaço vetorial tal que

$$
T_{e} G=\mathfrak{h} \oplus \mathfrak{h}^{\prime}
$$

e

$$
\begin{aligned}
\psi: \mathfrak{h} \oplus \mathfrak{h}^{\prime} & \longmapsto G \\
\left(X, X^{\prime}\right) & \longmapsto \exp (X) \cdot \exp \left(X^{\prime}\right)
\end{aligned}
$$

Então, existe vizinhança de $(0,0)$ tal que $\psi$ é difeomorfismo restrito a tal vizinhança.

Prova: Para demonstrar a afirmação 1.68, vamos derivar $\psi$ :

$$
\begin{aligned}
D_{1} \psi_{(0,0)}(X, 0) & =d \exp _{0} X \\
& =X \\
D_{2} \psi_{(0,0)}\left(0, X^{\prime}\right) & =d \exp _{0} X^{\prime} \\
& =X^{\prime}
\end{aligned}
$$

Disso temos que $D \psi_{0}=I$ e, pelo teorema da função inversa, $\psi$ é difeomorfismo em uma vizinhança de $(0,0)$.

Afirmação 1.69. Existe uma vizinhança $\mathcal{V}^{\prime}$ de $0 \mathrm{em} \mathfrak{h}^{\prime}$ tal que $\exp \left(X^{\prime}\right)$ não pertence a $H$, para $X^{\prime} \in V^{\prime}$.

Prova: Suponha por absurdo que exista sequência $\tilde{X}_{i} \longrightarrow 0$ tal que $\tilde{X}_{i} \in \mathfrak{h}^{\prime}$ e $\exp \left(\tilde{X}_{i}\right) \in H$. Defina $t_{i}=\left\|\tilde{X}_{i}\right\| \mathrm{e}$

$$
X_{i}:=\frac{\tilde{X}_{i}}{t_{i}}
$$


Como $X_{i}$ é uma sequência em um conjunto compacto, podemos tomar uma subsequência convergente (vamos denotar com o mesmo índice $i) X_{i} \longrightarrow X, t_{i} \longrightarrow 0$ e $\exp \left(t_{i} X_{i}\right) \in H$. Pela afirmação 1.66, $X \in \mathfrak{h}$, o que contraria $\mathfrak{h} \cap \mathfrak{h}^{\prime}=\{0\}$

Afirmação 1.70. Existe uma vizinhança $\mathcal{U}$ de 0 em $T_{e} G$ tal que

$$
H \cap \exp (\mathcal{U})=\exp (\mathfrak{h} \cap \mathcal{U})
$$

Note que, sendo a equação 1.11 verdadeira, temos que $H$ é uma variedade mergulhada em uma vizinhança de $e$. Como $H$ é grupo, $H$ é uma variedade mergulhada, e portanto $H$ é um subgrupo mergulhado. Segue da proposição 1.12 que $H$ é subgrupo de Lie mergulhado de $G$.

Basta então provar que a equação 1.11 é verdadeira:

Prova: Segue da construção de $\mathfrak{h}$ que

$$
H \cap \exp (\mathcal{U}) \supset \exp (\mathfrak{h} \cap \mathcal{U})
$$

Escolha $\mathcal{U}$ vizinhança de 0 em $T_{e} G$ de maneira que:

a) $\left.\exp \right|_{\mathcal{U}}$ é difeomorfismo

b) $\left.\psi\right|_{\mathcal{U}}$ é difeomorfismo (conforme a afirmação 1.68)

c) $\left(\mathcal{U} \cap \mathfrak{h}^{\prime}\right) \subset \mathcal{V}^{\prime}$, conforme a afirmação 1.69).

Seja $a \in H \cap \exp (\mathcal{U})$. Por b), existem únicos $X \in \mathfrak{h}$ e $X^{\prime} \in \mathfrak{h}^{\prime} \operatorname{com} a=\exp (X) \cdot \exp \left(X^{\prime}\right)$, e assim $\exp \left(X^{\prime}\right)=[\exp (X)]^{-1} \cdot a \in H$. Segue da afirmação 1.69 que $X^{\prime}=0$, ou seja, $a=\exp (X), \operatorname{com}$ $X \in \mathfrak{h}$. Como $a$ foi tomado arbitrário, segue a equação 1.11 .

Corolário 1.71. Sejam $G, H$ grupos de Lie e $\varphi: G \longrightarrow H$ um homomorfismo contínuo. Então, $\varphi$ é suave.

Demonstração: Considere o gráfico $K=\{(g, \varphi(g)): g \in G\}$. $K$ é um subgrupo algébrico fechado de $G \times H$, então por consequência do teorema 1.65, $K$ é subgrupo de Lie mergulhado de $G \times H$. Tomando a inclusão $i: K \longrightarrow G \times H$, e as projeções $\pi_{1}, \pi_{2}$ respectivamente na primeira e segunda componente, temos que $\pi_{1} \circ i$ é aplicação de posto constante, pois $\pi_{1} \circ i \circ L^{K}=L^{G} \circ \pi_{1} \circ i$. $\mathrm{O}$ fato de $K$ ser gráfico em conjunto com o teorema do posto garante que $\pi_{1} \circ i$ é imersão.

Necessariamente, $\operatorname{dim}(K)=\operatorname{dim}(G)$, pois se não $\pi_{1} \circ i(K)$ teria medida nula em $G$, contrariando $\pi_{1} \circ i(K)=G$. Usando o teorema da função inversa, temos que $\pi_{1} \circ i$ é difeomorfismo local e, como 
$\pi_{1} \circ i$ é bijeção, é um difeomorfismo global. Por fim, $\varphi=\pi_{2} \circ\left(\pi_{1} \circ i\right)^{-1}$, e portanto uma aplicação suave.

Observação 1.72. Se $\varphi: G \longrightarrow H$ é um homomorfismo de Lie, então $\operatorname{ker}(\varphi)=\varphi^{-1}(e)$ é subgrupo de Lie fechado.

Observação 1.73. Seja $G$ um grupo de Lie conexo e

$$
\mathcal{Z}(G)=\{g \in G: g b=b g, \quad \forall b \in G\}
$$

Então, $\mathcal{Z}(G)=\operatorname{ker}(A d)$. De fato, se $g \in \mathcal{Z}(G)$ evidentemente Ad $(g)=I$. Tomemos $g \in \operatorname{ker}(\operatorname{Ad})$, usando a equação 1.9 temos:

$$
g \exp (t X) g^{-1}=\exp (t X)
$$

E portanto $g$ comuta com todos os elementos numa vizinhança de $e$ onde exp está definida. O resultado segue da proposição 1.50 .

\subsection{Fatos de Geometria Riemanniana}

A partir de agora, vamos envolver em nosso estudo alguns conceitos de geometria riemanniana. Mas, para não desviar o objetivo central deste capítulo, apenas faremos uma listagem das definições e resultados a serem utilizados. Para um estudo mais cuidadoso, recomendamos a leitura do segundo e terceiro capítulo de [dC2].

Definição 1.74. Seja $M$ uma variedade riemanniana com métrica $\langle$,$\rangle . Uma conexão riemanniana$ (compatível com a métrica) é uma aplicação:

$$
\nabla: \Xi(M) \times \Xi(M) \longrightarrow \Xi(M)
$$

satisfazendo:

a) $\nabla_{f X+Y} Z=f \nabla_{X} Z+\nabla_{Y} Z$

b) $\nabla_{X}(Y+Z)=\nabla_{X} Y+\nabla_{X} Z$

c) $\nabla_{X} f Y=(X f) Y+f \nabla_{X} Y$

d) $X\langle Y, Z\rangle=\left\langle\nabla_{X} Y, Z\right\rangle+\left\langle Y, \nabla_{X} Z\right\rangle$

e) $[X, Y]=\nabla_{X} Y-\nabla_{Y} X$

Observação 1.75 (Fórmula da Conexão).

$$
2\left\langle\nabla_{Y} X, Z\right\rangle=X\langle Y, Z\rangle-Z\langle X, Y\rangle+Y\langle Z, X\rangle-\langle[X, Z], Y\rangle-\langle[Y, Z], X\rangle-\langle[X, Y], Z\rangle
$$


Note que esta fórmula exibe o candidato natural a conexão em uma variedade riemanniana e além disso mostra que esta está unicamente determinada pela métrica. (cf. [dC2], pg. 61)

Definição 1.76 (Derivada Covariante). Seja $\tilde{V}$ um campo em uma variedade $M$ e $\alpha: I \longrightarrow M$ uma curva suave. Considere $V(t)=\tilde{V} \circ \alpha(t)$. Definimos a derivada covariante de $V$ ao longo de $\alpha$ como:

$$
\frac{\nabla}{d t} V:=\nabla_{\alpha^{\prime}(t)} \tilde{V}
$$

Seja $V(t)$ um campo ao longo de uma curva $\alpha: I \longrightarrow M$.

$$
\nabla_{\alpha^{\prime}(t)} V:=\nabla_{\alpha^{\prime}(t)} \widetilde{V}
$$

onde $\widetilde{V}$ é uma extensão de $V$ na vizinhança de $\alpha$.

Definição 1.77. Uma curva $\alpha: I \longrightarrow M$ é geodésica se

$$
\nabla_{\alpha^{\prime}(t)} \alpha^{\prime}(t)=0
$$

Proposição 1.78 (cf. [dC2], pg. 58). Seja $\gamma$ uma curva. Dado $v \in T_{\gamma(0)} M$, existe um único campo $V$ ao longo de $\gamma \operatorname{com} \nabla_{\gamma^{\prime}(t)} V=0$. $V$ é chamado transporte paralelo de $v$.

Proposição 1.79 (cf. [dC2], pg. 70). Dado $p \in M$, existe $\varepsilon>0$ tal que, dado $X \in \mathcal{B}_{\varepsilon}(0) \subset T_{p} M$, existe uma única curva $\alpha:[-1,1] \longrightarrow M \operatorname{com} \alpha(0)=p$ e $\alpha^{\prime}(0)=X$.

Definição 1.80 (Exponencial Riemanniana).

$$
\begin{aligned}
\exp _{p}: \mathcal{B}_{\varepsilon}(0) & \longrightarrow M \\
x & \longmapsto \alpha_{X}(1)
\end{aligned}
$$

Observação 1.81. Se $\alpha$ é geodésica, então $\left\|\alpha^{\prime}\right\|$ é constante.

Proposição 1.82 (cf. [dC2], pg. 73, 77). Dado $p \in M$, existe $\mathcal{B}_{\varepsilon}(0) \subset T_{p} M$ tal que:

a) $\left.\exp _{p}\right|_{\mathcal{B}_{\varepsilon}(0)}$ é difeomorfismo, e d $\exp _{p}=I$

b) $\alpha(t)=\exp _{p}(t X)$ minimiza a distância entre $p$ e $\exp (X)$

Teorema 1.83 (cf. [dC2], pg. 80). Seja $p \in M$. Então, existe $\varepsilon>0$ e vizinhança $\mathcal{W}$ de $p$ sendo que:

a) Para $x \in \mathcal{W}, \exp _{x}: \mathcal{B}_{\varepsilon}(0) \longrightarrow M$ é difeomormismo.

b) $\exp _{x}(\mathcal{W}) \supset \mathcal{W}, \forall x \in \mathcal{W}$.

c) Dado $X, Y \in \mathcal{W}$, a geodésica minimimizante ligando $X$ a $Y$ está contida em $\mathcal{W}$.

A seguir, enunciamos o teorema de Hopf-Rinow, cuja demonstração pode ser vista em [dC2], pg. 162 . 
Teorema 1.84 (Hopf-Rinow). Seja $M$ uma variedade e $p \in M$. As afirmações abaixo são equivalentes:

1. $\exp _{p}: T_{p} M \longrightarrow M$, ou seja, a exponencial riemanniana em $p$ está globalmente definida

2. Todo fechado e limitado em $M$ é compacto

3. $M$ é espaço métrico completo

4. A exponencial riemanniana $\exp _{x}: T_{x} M \longrightarrow M$ está definida em todo $x \in M$.

Uma variedade riemanniana é completa se atende a alguma das asserções acima (e portanto todas).

Definição 1.85. Em uma variedade riemanniana, definimos a curvatura por:

$$
R(X, Y) Z:=\nabla_{[X, Y]} Z-\nabla_{X} \nabla_{Y} Z+\nabla_{Y} \nabla_{X} Z
$$

E curvatura de Ricci por:

$$
\operatorname{Ric}(X, Y):=\operatorname{tr} R(X, \bullet) Y
$$

A demonstração do teorema de Bonnet-Myers abaixo pode ser conferida em [dC2], pg. 221.

Teorema 1.86 (Bonnet-Myers). Seja $M$ uma variedade riemanniana completa. Suponha que $\operatorname{Ric}(X, Y) \geq \frac{1}{\lambda}\langle X, Y\rangle$. Então

a) $M$ é compacta

b) $\operatorname{diam}(M) \leq \pi \sqrt{\frac{n-1}{\lambda}}$, sendo $n$ a dimensão de $M$

c) $\pi_{1}(M)$ é finito

d) O recobrimento $\widetilde{M}$ é compacto

\subsection{Métricas Bi-invariantes}

Definição 1.87 (Métrica Invariante à Esquerda). Seja $G$ um grupo de Lie. Uma métrica $\langle$,$\rangle é$ invariante à esquerda se $L_{g}$ é isometria, $\forall g \in G$. Isto quer dizer:

$$
\left\langle\left(d L_{g}\right)_{h} U,\left(d L_{g}\right)_{h} V\right\rangle_{g h}=\langle U, V\rangle_{h}, \quad \forall g, h \in G, \forall U, V \in T_{h} G
$$

Analogamente, definimos métrica invariante à direita.

Dada uma métrica em $T_{e} G$, podemos construir uma métrica invariante à esqueda em $G$ por:

$$
\langle V, W\rangle_{g}:=\left\langle d L_{g^{-1}} V, d L_{g^{-1}} W\right\rangle_{e}
$$

onde $g$ é qualquer elemento em $G$ e $V, W$ são vetores em $T_{g} G$. 
Definição 1.88. Uma $k$-forma $\omega$ é invariante à esquerda se $L_{g}^{*} \omega=\omega$

Analogamente, definimos $k$-forma invariante à direita.

Dado um $k$-tensor alternado em $T_{e} G$, definimos uma $k$-forma invariante à esquerda como:

$$
\omega_{g}\left(v_{1}, \ldots, v_{k}\right)=\omega_{e}\left(d L_{g^{-1}} v_{1}, \ldots, d L_{g^{-1}} v_{k}\right)
$$

Definição 1.89. Uma métrica é bi-invariante se $L_{g}$ e $R_{g}$ são isometrias, $\forall g \in G$.

Proposição 1.90. Seja $G$ um grupo de Lie compacto. Então G admite métrica bi-invariante.

Demonstração: Seja $\omega$ uma $n$-forma invariante à direita $(\mathrm{n}=\operatorname{dim} G)$ e $\langle$,$\rangle uma métrica invariante$ à direita. Defina:

$$
\leqslant V, W \geqslant_{x}:=\int_{G}\left\langle d L_{g} V, d L_{g} W\right\rangle_{g x} \omega
$$

Mostremos que:

$1 . \leqslant, \geqslant$ é invariante à esquerda.

$$
\begin{aligned}
\leqslant d L_{h} V, d L_{h} W \geqslant_{h x} & =\int_{G}\left\langle d L_{g}\left(d L_{h} V\right), d L_{g}\left(d L_{h} W\right)\right\rangle_{g(h x)} \omega \\
& =\int_{G}\left\langle d L_{g h} V, d L_{g h} W\right\rangle_{(g h) x} \omega
\end{aligned}
$$

Vamos fixar $V, W, x$ e denotar:

$$
f(g)=\left\langle d L_{g} V, d L_{g} W\right\rangle_{g x}
$$

Assim, usando que $R_{h}^{*} \omega=\omega$ e aplicando mudança de variável, temos:

$$
\begin{aligned}
\int_{G}\left\langle d L_{g h} V, d L_{g h} W\right\rangle_{(g h) x} \omega & =\int_{G} f(g h) \omega \\
& =\int_{G} R_{h}^{*}(f \omega) \\
& =\int_{G} f \omega \\
& =\int_{G}\left\langle d L_{g} V, d L_{g} W\right\rangle_{g x} \omega \\
& =\leqslant V, W \geqslant x
\end{aligned}
$$


$2 . \leqslant, \geqslant$ é invariante à direita.

$$
\begin{aligned}
\leqslant d R_{h} V, d R_{h} W \geqslant_{x h} & =\int_{G}\left\langle d L_{g}\left(d R_{h} V\right), d L_{g}\left(d R_{h} W\right)\right\rangle_{g(x h)} \omega \\
& =\int_{G}\left\langle d R_{h} d L_{g} V, d R_{h} d L_{g} W\right\rangle_{(g x) h} \omega \\
& =\int_{G}\left\langle d L_{g} V, d L_{g} W\right\rangle_{g x} \omega \\
& =\leqslant V, W \geqslant_{x}
\end{aligned}
$$

Teorema 1.91. Seja $G$ um grupo de Lie conexo com métrica bi-invariante. Para cada a $\in G$, defina

$$
\begin{aligned}
I^{a} & : G \longrightarrow G \\
\quad g & \longmapsto a g^{-1} a
\end{aligned}
$$

Então:

a) $I^{a}$ é uma isometria que inverte geodésica, isto é, se $\alpha$ é geodésica com $\alpha(0)=a, I^{a}(\alpha(t))=$ $\alpha(-t)$.

b) A geodésica $\alpha$, com $\alpha(0)=e$ e $\alpha^{\prime}(0)=X$ é o subgrupo a um parâmetro $\exp (t X)$.

\section{Demonstração:}

a) Veja que $d_{I}^{e}=-I$, e portanto uma isometria, e $I^{e}(\alpha(t))=\alpha(t)^{-1}$ sendo $\frac{d}{d t} I^{e}(\alpha(t))=-\alpha^{\prime}(t)$. Logo, $I^{e}$ é uma isometria que inverte geodésica.

Agora, tomando $a \in G$ arbitrário, temos $I^{a}=R_{a} \circ I^{e} \circ L_{a^{-1}}$; é isometria por ser composta de isometria, e inverte geodésica, já que $I^{e}$ inverte geodésica.

b) Vamos provar por indução em $n$ que $\alpha(n t)=\alpha(t)^{n}, \forall n \in \mathbb{N}$ :

De fato, tomando $u, t \in \mathbb{R}$, temos:

$$
\begin{aligned}
\alpha(t) \cdot \alpha(u) \cdot \alpha(t) & =I^{\alpha(t)} \circ I^{e}(\alpha(u)) \\
& =I^{\alpha(t)}(\alpha(-u))
\end{aligned}
$$

Fazendo $\tilde{\alpha}(u)=\alpha(u+t)$, continuamos:

$$
\begin{aligned}
I^{\alpha(t)}(\alpha(-u)) & =I^{\tilde{\alpha}(0)} \tilde{\alpha}(-u-t) \\
& =\tilde{\alpha}(u+t) \\
& =\alpha(u+2 t)
\end{aligned}
$$

Agora, tomando $u=0$, resulta:

$$
\alpha(2 t)=\alpha(t)^{2}
$$


Supondo válido para $n$, segue:

$$
\begin{aligned}
\alpha(t)^{n+1} & =\alpha(t)^{n} \cdot \alpha(t) \\
& =\alpha(n t) \cdot \alpha(t) \\
& =\alpha(n t+t) \\
& =\alpha((n+1) t)
\end{aligned}
$$

E portanto a igualdade é válida para todo $n \in \mathbb{N}$.

Mostremos que $\left.\alpha\right|_{\mathbb{Q}(-\varepsilon, \varepsilon)}$ é um homomorfismo.

Sejam $a, b \in \mathbb{Q} \cap(-\varepsilon, \varepsilon)$, e tome um racional $t$ de maneira que $a=n t$ e $b=m t$, com $n, m$ números inteiros. Assim:

$$
\begin{aligned}
\alpha(a+b) & =\alpha((n+m) t) \\
& =\alpha(t)^{n+m} \\
& =\alpha(t)^{n} \cdot \alpha(t)^{m} \\
& =\alpha(n t) \cdot \alpha(m t) \\
& =\alpha(a) \cdot \alpha(b)
\end{aligned}
$$

Usando a continuidade de $\alpha$, resulta que $\left.\alpha\right|_{(-\varepsilon, \varepsilon)}$ é homomorfismo local. Para verificar que $\alpha$ é um homomorfismo de Lie, basta notar que $\left.\alpha\right|_{(-\varepsilon, \varepsilon)}$ é segmento de geodésica contida no subgrupo $\exp (t X)=\beta(t)$, e portanto $L_{\alpha\left(\frac{\varepsilon}{2}\right)} \alpha$ é um segmento de geodésica contido em $\beta$. Dado um certo $I \subset \mathbb{R}$, podemos repetir este processo de forma que $\alpha$ se estenda em $I$.

Corolário 1.92. A exponencial de Lie em um grupo de Lie compacto é sobrejetora.

Demonstração: Se $G$ é compacto, então $G$ admite métrica bi-invariante (proposição 1.90) e, para tal métrica, $\exp (X)=\exp _{e}(X)$ (teorema 1.91). Pelo teorema 1.84, $G$ é variedade riemanniana completa, e portanto $\exp =\exp _{e}: T_{e} G \longrightarrow G$ é sobrejetor.

Proposição 1.93. Sejam $G$ grupo de Lie com métrica bi-invariante, $X, Y, Z$ campos invariantes a esquerda. Então:
(a) $\langle[X, Y], Z\rangle=-\langle Y,[X, Z]\rangle$
(b) $\nabla_{X} Y=\frac{1}{2}[X, Y]$
(c) $R(X, Y) Z=\frac{1}{4}[[X, Y], Z]$
(d) $\langle R(X, Y) X, Y\rangle=\frac{1}{4}\langle[X, Y],[X, Y]\rangle$. 
Em particular, a curvatura seccional

$$
K(\sigma)=\frac{\langle R(X, Y) X, Y\rangle}{\sqrt{\|X\|^{2}\|Y\|^{2}-\langle X, Y\rangle^{2}}} \geq 0
$$

\section{Demonstração:}

(a) Derivando a expressão:

$$
\left\langle\operatorname{Ad}\left(e^{t X}\right) Y, \operatorname{Ad}\left(e^{t X}\right) Z\right\rangle=\langle Y, Z\rangle
$$

temos que (vide equação 1.10 e lema 1.63):

$$
\langle[X, Y], Z\rangle+\langle Y,[X, Z]\rangle=0
$$

(b) Segue diretamente da fórmula da conexão (observação 1.75), com uso do item a) desta proposição.

(c)

$$
\begin{aligned}
R(X, Y) Z & =\nabla_{[X, Y]} Z-\nabla_{X} \nabla_{Y} Z+\nabla_{Y} \nabla_{X} Z \\
& =\frac{1}{2}[[X, Y], Z]-\frac{1}{2} \nabla_{X}[Y, Z]+\frac{1}{2} \nabla_{Y}[X, Z] \\
& =\frac{1}{2}[[X, Y], Z]-\frac{1}{4}[X,[Y, Z]]+\frac{1}{4}[Y,[X, Z]] \\
& =\frac{1}{4}[[X, Y], Z]+\left(\frac{1}{4}[[X, Y], Z]+\frac{1}{4}[[Z, X], Y]+\frac{1}{4}[[Y, Z], X]\right) \\
& =\frac{1}{4}[[X, Y], Z]
\end{aligned}
$$

devido à igualdade de Jacobi (definição 1.13).

(d)

$$
\begin{aligned}
\langle R(X, Y) X, Y\rangle & =\frac{1}{4}\langle[[X, Y], X], Y\rangle \\
& =-\frac{1}{4}\langle[X,[X, Y]], Y\rangle \\
& =\frac{1}{4}\langle[X, Y],[X, Y]\rangle
\end{aligned}
$$

fazendo uso do item (a) dessa proposição.

\subsection{Forma de Killing}

Para completar o estudo de métricas bi-invariantes em grupos de Lie, introduzimos as formas de Killing por meio da qual estabeleceremos condições para a compacidade de um grupo de Lie. 
Definição 1.94 (Forma de Killing). Dados $X, Y \in \mathfrak{g}$, onde $G$ é grupo de Lie, a forma de Killing é definida por

$$
B(X, Y):=\operatorname{tr}(\operatorname{ad}(X) \cdot \operatorname{ad}(Y))
$$

Observação 1.95. B é bilinear e simétrica

Definição 1.96. $\mathfrak{g}$ é semi-simples se $B$ é não-degenerada.

Proposição 1.97 .

$$
B(X, Y)=B(A d(g) X, A d(g) Y)
$$

Demonstração: Seja $\varphi: \mathfrak{g} \longrightarrow \mathfrak{g}$ um automorfismo de álgebras de Lie. Então:

$$
\operatorname{ad}(\varphi(X)) \varphi(Y)=\varphi \operatorname{ad}(X) Y
$$

E, consequentemente

$$
\operatorname{ad}(\varphi(X))=\varphi \operatorname{ad}(X) \varphi^{-1}
$$

Daí:

$$
\begin{aligned}
B(\varphi(X), \varphi(Y)) & =\operatorname{trad}(\varphi(X)) \varphi(Y)) \\
& =\operatorname{tr}\left(\varphi \operatorname{ad}(X) \varphi^{-1}\right) \\
& =\operatorname{tr}(\operatorname{ad}(X) \operatorname{ad}(Y)) \\
& =B(X, Y)
\end{aligned}
$$

Como Ad $(g)$ é um automorfismo de Lie, o resultado é válido.

Corolário 1.98. Suponha $\mathfrak{g}$ semi-simples e B negativo-definida. Então-B é métrica bi-invariante.

Observação 1.99. Sendo $G$ um grupo de Lie com métrica bi-invariante, então:

$$
\begin{aligned}
\operatorname{Ric}(X, Y) & =\operatorname{Ric}(X, Y):=\operatorname{tr} R(X, \bullet) Y \\
& =\operatorname{tr} \frac{1}{4}[[X, \bullet], Y] \\
& =-\frac{1}{4} \operatorname{tr}[[\bullet, X], Y] \\
& =-\frac{1}{4} \operatorname{tr}[Y,[X, \bullet]] \\
& =-\frac{1}{4} B(Y, X) \\
& =-\frac{1}{4} B(X, Y)
\end{aligned}
$$


E portanto segue a fórmula

$$
\operatorname{Ric}(X, Y)=-\frac{1}{4} B(X, Y)
$$

Em particular, a curvatura de Ricci independe da métrica bi-invariante.

Teorema 1.100. Seja $G$ um grupo de Lie conexo semi-simples. G é compacto se, e somente se, $B$ é negativo-definida.

Demonstração: $(\Leftarrow)$ Suponha $B$ negativo-definida, então $-B$ é métrica bi-invariante. Assim, $(G,-B)$ é uma variedade Riemanniana completa (teorema 1.84), onde a equação 1.13 é satisfeita. Segue do teorema de Bonnet-Myers (teorema 1.86) que $G$ é compacto.

$(\Rightarrow)$ Suponha $G$ compacto. Pela proposição 1.90, $G$ admite métrica bi-invariante e assim:

$$
\begin{aligned}
B(X, X) & =\operatorname{tr} \operatorname{ad}(X) \operatorname{ad}(X) \\
& =\sum_{i=1}^{n}\left\langle\operatorname{ad}(X) \operatorname{ad}(X) e_{i}, e_{i}\right\rangle \\
& =-\sum_{i=1}^{n}\left\langle\operatorname{ad}(X) e_{i}, \operatorname{ad}(X) e_{i}\right\rangle \\
& =-\sum_{i=1}^{n}\left\|\operatorname{ad}(X) e_{i}\right\| \quad \leq 0
\end{aligned}
$$

Na segunda passagem da conta acima, fizemos uso do item a) da proposição 1.93 e do lema 1.63

Como $B$ é semi-simples, $B(X, X)<0$ e portanto negativo-definida.

\subsection{Decomposição em Ideais Simples}

Finalizamos este capítulo com um resultado importante de decomposição, por meio do qual é possível enxergar um grupo de Lie como o produto de retas com grupos compactos. Sob algumas condições, conseguimos assim garantir a existência de uma única métrica bi-invariante, induzida da forma de Killing.

Definição 1.101 (Ideal). Uma subálgebra de Lie $\mathfrak{h}$ de uma álgebra de Lie $\mathfrak{g}$ é chamada ideal se $[X, Y] \in \mathfrak{h}, \forall X \in \mathfrak{h}, Y \in \mathfrak{g}$.

Quando os únicos ideais de $\mathfrak{h}$ forem $\{0\}$ e $\mathfrak{h}$, então o ideal será simples.

Teorema 1.102. Seja $\mathfrak{g}$ uma álgebra de Lie com métrica bi-invariante, isto é, $\langle[X, Y], Z\rangle=$ $-\langle Y,[X, Z]\rangle$.

(a) $\mathfrak{g}$ é igual à soma direta ortogonal $\mathfrak{g}_{1} \oplus \cdots \oplus \mathfrak{g}_{n}$ de ideais simples.

(b) Se $\widetilde{G}$ é o grupo de Lie conexo e simplesmente conexo com álgebra de Lie $\mathfrak{g}$, então $\widetilde{G}$ é isomorfo a um produto de subgrupos normais de Lie $G_{1} \times \cdots \times G_{n}$ tais que: 
(b1) $\mathfrak{g}_{i}$ é algebra de Lie de $G_{i}$.

(b2) Se $\mathfrak{g}_{i}$ é comutativa, então $G_{i}=\mathbb{R}$. Se $\mathfrak{g}_{i}$ não é comutativa, então $G_{i}$ é compacto.

\section{Demonstração:}

(a) Basta mostrar que se $\mathfrak{h}$ é um ideal, $\mathfrak{h}^{\perp}$ é também um ideal. Seja $X \in \mathfrak{h}^{\perp}, Y \in \mathfrak{g}$ e $Z \in \mathfrak{h}$ :

$$
\begin{aligned}
\langle[X, Y], Z\rangle & =-\langle[Y, X], Z\rangle \\
& =\langle X,[Y, Z]\rangle \\
& =0
\end{aligned}
$$

e portanto $[X, Y] \in \mathfrak{h}^{\perp}$.

(b) Pelo $3^{o}$ Teorema de Lie (teorema 1.30), dado $\mathfrak{g}_{i}$ existe um único subgrupo de Lie conexo, simplesmente conexo $G_{i}$ com álgebra de Lie $\mathfrak{g}_{i}$. Assim, $G_{1} \times \ldots \times G_{n}$ é grupo de Lie conexo, simplesmente conexo, com álgebra de Lie $\mathfrak{g}_{1} \oplus \ldots \oplus \mathfrak{g}_{n}=\mathfrak{g}$. Pela unicidade dada pelo $3^{o}$ teorema de Lie, $G_{1} \times \ldots \times G_{n} \cong \tilde{G}$ do enunciado.

Antes de provar que os subgrupos $G_{i}$ são normais observamos que, sendo $\mathfrak{g}_{i}$ comutativo e simples, então $\mathfrak{g}_{i} \cong \mathbb{R}$. Daí, como $G_{i}$ é conexo e simplesmente conexo, segue que $G_{i} \cong \mathbb{R}$.

No caso em que $\mathfrak{g}_{i}$ não é comutativo, observamos que não pode existir $X \in \mathfrak{g}_{i}, X \neq 0$ tal que $[X, Y]=0, \forall Y \in \mathfrak{g}_{i}$, pois se existisse $\{X\}$ seria um ideal de $\mathfrak{g}_{i}$, o que contraria nossa hipótese. Usando o argumento da demonstração da proposição $1.100(\Rightarrow)$, temos que a forma de Killing de $\mathfrak{g}_{i}$ é negativo-definida. Pela mesma proposição, concluimos que $G_{i}$ é compacto.

Lema 1.103. $\mathfrak{g}_{i}$ ideal implica que $G_{i}$ é subgrupo normal

Prova: Tomando $X \in \mathfrak{g}_{i}, Y \in \mathfrak{g}$, temos $[X, Y] \in \mathfrak{g}_{i}$. Usando a observação 1.62, temos que:

$$
\begin{aligned}
\operatorname{Ad}(\exp (Y)) X & =\exp (\operatorname{ad}(Y)) X \\
& =\sum_{k=1}^{\infty} \frac{\operatorname{ad}(Y)^{k}}{k !} X \in \mathfrak{g}_{i}
\end{aligned}
$$

Por outro lado, pela equação 1.9

$$
\exp (Y) \exp (X) \exp (Y)^{-1}=\exp (\operatorname{Ad}(\exp (Y)) X)
$$


Isto implica

$$
\exp (Y) \exp (X) \exp (Y)^{-1} \in G_{i}
$$

Concluímos o resultado a partir da proposição 1.50

Proposição 1.104. Seja G um grupo de Lie compacto, com métrica bi-invariante, cuja álgebra de Lie $\mathfrak{g}$ seja simples. Então, a métrica bi-invariante é única, a menos de multiplicação por constante, e além disso:

$$
\operatorname{Ric}(X, Y)=k\langle X, Y\rangle
$$

Demonstração: Seja $\langle$,$\rangle uma métrica bi-invariante dada, e ( , ) outra métrica bi-invariante.$ Então, existe uma matriz simétrica $A$ positivo-definida tal que:

$$
(X, Y)=\langle A X, Y\rangle
$$

Afirmação 1.105.

$$
A \operatorname{ad}(X)=\operatorname{ad}(X) A
$$

Prova:

$$
\begin{aligned}
\langle A \operatorname{ad}(X) Y, Z\rangle & =(\operatorname{ad}(X) Y, Z) \\
& =-(Y, \operatorname{ad}(X) Z) \\
& =-\langle A Y, \operatorname{ad}(X) Z\rangle \\
& =\langle\operatorname{ad}(X) A Y, Z\rangle
\end{aligned}
$$

Afirmação 1.106. ad $(X)$ deixa os auto-espaços de $A$ invariantes, isto é, os auto-espaços são ideais.

Prova: Seja $V$ tal que $A V=\lambda V$. Então:

$$
\begin{aligned}
A \operatorname{ad}(X) V & =\operatorname{ad}(X) A V \\
& =\lambda \operatorname{ad}(X) V
\end{aligned}
$$


Como $\mathfrak{g}$ é simples, segue da afirmação 1.106 que $A=\lambda I$ e portanto:

$$
(X, Y)=\lambda\langle X, Y\rangle
$$

Além disso, como $G$ é compacto, segue da proposição 1.100 e corolário 1.98 que $-B$ é métrica bi-invariante, e então existe uma constante $k$ tal que $-B(X, Y)=4 k\langle X, Y\rangle$. Pela equação 1.13, resulta:

$$
\begin{aligned}
\operatorname{Ric}(X, Y) & =-\frac{1}{4} B(X, Y) \\
& =\frac{1}{4} 4 k\langle X, Y\rangle \\
& =k\langle X, Y\rangle
\end{aligned}
$$




\section{Capítulo 2}

\section{Ações Próprias}

Se antes efetuamos o estudo cuidadoso dos grupos de Lie, agora queremos estudar a ação deste grupo em uma variedade. Neste capítulo definimos ações e ações próprias, e um estudo inicial de fibrados é realizado afim de mostrar que o espaço de órbitas de uma ação livre e própria é variedade e ainda que $\pi: M \longrightarrow M / G$ é fibrado principal.

A seguir definimos subvariedade slice e mostramos que tal subvariedade sempre existe se a ação for própria. Além disso, construímos uma vizinhança tubular de uma órbita regular por meio da subvariedade slice.

As ações isométricas são então introduzidas, visto que ações isométricas próprias desempenham um papel fundamental nesta dissertação. Demonstramos alguns teoremas pertinentes a ações isométricas próprias e em seguida definimos representação isotrópica e representação slice.

O importante Teorema da Órbita Principal (dentre os mais relevantes deste trabalho) é demonstrado e finalizamos o capítulo apresentando tipos de órbitas e uma forma de estratificar uma variedade $M$ através das componentes conexas dos tipos de órbitas.

\subsection{Definições e Resultados Iniciais}

Aqui definimos ações de grupos de Lie em variedades (à esquerda e à direita) sempre exibindo exemplos para familiarizar o leitor com este conceito. Apresentamos também uma gama de resultados sobre ações e terminamos por definir ação própria, inclusive exibindo versões equivalentes para sua definição.

Definição 2.1 (Ação à Esquerda). Seja $G$ um grupo de Lie e $M$ uma variedade. A aplicação suave

$$
\mu: G \times M \longrightarrow M
$$

é uma ação à esquerda de $G$ em $M$ se:

a) $\mu(e, x)=x, \forall x \in M$

b) $\mu\left(g_{1}, \mu\left(g_{2}, x\right)\right)=\mu\left(g_{1} g_{2}, x\right), \forall g_{1}, g_{2} \in G$ e $\forall x \in M$.

Por simplicidade, denotaremos $g \cdot x=\mu(g, x)$.

Exemplo 2.2. Alguns exemplos de ação à esquerda são listados abaixo: 
1.

$$
\begin{aligned}
& H \times G \longrightarrow G \\
& (h, g) \longmapsto h \cdot g
\end{aligned}
$$

onde $H$ um subgrupo de Lie de $G$.

2.

$$
\begin{gathered}
S O_{3}(\mathbb{R}) \times \mathbb{R}^{3} \longrightarrow \mathbb{R}^{3} \\
(A, v) \longmapsto A v
\end{gathered}
$$

3.

$$
\begin{aligned}
& G \times G \longrightarrow G \\
& (a, b) \longmapsto a \cdot b \cdot a^{-1}
\end{aligned}
$$

4 .

$$
\begin{aligned}
A d: G \times \mathfrak{g} & \longrightarrow \mathfrak{g} \\
(g, V) & \longmapsto A d(g) V
\end{aligned}
$$

De modo análogo, podemos definir ação à direita, como fazemos abaixo:

Definição 2.3 (Ação à Direita). Seja $G$ um grupo de Lie e $M$ uma variedade. A aplicação suave

$$
\mu: M \times G \longrightarrow M
$$

é uma ação à direita de $G$ em $M$ se:

a) $\mu(x, e)=x, \forall x \in M$

b) $\mu\left(\mu\left(x, g_{1}\right), g_{2}\right)=\mu\left(x, g_{1} g_{2}\right), \forall g_{1}, g_{2} \in G$ e $\forall x \in M$.

Por simplicidade, denotaremos $x \cdot g=\mu(x, g)$.

Exemplo 2.4. Alguns exemplos de ação à direita:

1. Considerando novamente $H$ subgrupo de Lie de $G$, temos a ação

$$
\begin{aligned}
& G \times H \longrightarrow G \\
& (g, h) \longmapsto g \cdot h
\end{aligned}
$$

2. Seja $V$ um espaço vetorial $n$-dimensional e $B(V)$ o espaço dos referenciais de $V$, ou seja, dos isomorfismos lineares $T: \mathbb{R}^{n} \longrightarrow V$. Uma ação à direita de $G L_{n}(\mathbb{R})$ em $B(V)$ é dada por

$$
\begin{gathered}
B(V) \times G L_{n}(\mathbb{R}) \longrightarrow B(V) \\
(Z, g) \longmapsto Z \circ g
\end{gathered}
$$

Observação 2.5. Considere $M$ uma variedade e $B(T M)$ seu fibrado de referenciais, isto é, $B(T M)=$ $\cup_{p \in M} B\left(T_{p} M\right)$. Temos uma ação à direita dada por:

$$
\begin{gathered}
B(T M) \times G L_{n}(\mathbb{R}) \longrightarrow B(T M) \\
(Z, g) \longmapsto Z \circ g
\end{gathered}
$$


Observação 2.6. Se $\mu: G \times M \longrightarrow M$ é ação à esquerda, então $\widetilde{\mu}: M \times G \longrightarrow M$ definida por $\widetilde{\mu}(x, g)=\mu\left(g^{-1}, x\right)$ é ação à direita.

De fato, $\widetilde{\mu}(x, e)=\mu(e, x)=x \quad \mathrm{e}$

$$
\begin{aligned}
\widetilde{\mu}\left(\widetilde{\mu}\left(x, g_{1}\right), g_{2}\right) & =\mu\left(g_{2}^{-1}, \mu\left(g_{1}^{-1}, x\right)\right) \\
& =\mu\left(g_{2}^{-1} g_{1}^{-1}, x\right) \\
& =\mu\left(\left(g_{1} g_{2}\right)^{-1}, x\right) \\
& =\widetilde{\mu}\left(x, g_{1} g_{2}\right)
\end{aligned}
$$

Definição 2.7. Dada uma ação à esquerda $G \times M \longrightarrow M$, definimos:

1. Grupo de Isotropia de $x \in M$ como

$$
G_{x}=\{g \in G: g \cdot x=x\}
$$

2. Órbita de $x \in M$ como

$$
G(x)=\{g \cdot x: g \in G\}
$$

Tal ação será chamada:

3. Transitiva se, dados $x, y \in M$, existir $g \in G$ tal que $g \cdot x=y$.

4. Efetiva se o fato de $g \cdot x=x$, para todo $x \in M$ implicar $g=e$. Em outros termos, se $\cap_{x \in M} G_{x}=\{e\}$.

5. Livre, se $G_{x}=\{e\}, \forall x \in M$.

Proposição 2.8. Seja $G \times M \longrightarrow M$ ação à esquerda.

i) $G(x) \cap G(y) \neq \emptyset \Rightarrow G(x)=G(y)$

ii) $G_{g \cdot x}=g \cdot G_{x} \cdot g^{-1}$

\section{Demonstração:}

i) Suponha que exista $z \in G(x) \cap G(y)$. Então, $z=g_{1} \cdot x=g_{2} \cdot y$, sendo $g_{1}, g_{2} \in G$. Portanto, $x=\left(g_{1}^{-1} g_{2}\right) y$, o que implica $x \in G(y)$. De modo análogo, mostramos que $y \in G(x)$, resultando a igualdade das órbitas.

ii) Vamos mostrar as inclusões:

- $g \cdot G_{x} \cdot g^{-1} \subset G_{g \cdot x}$

Seja $h \in g \cdot G_{x} \cdot g^{-1}$. Então $h=g l g^{-1}$, sendo $l \cdot x=x$, e podemos efetuar: 


$$
h \cdot(g \cdot x)=\left(g l g^{-1}\right)(g \cdot x)=g(l \cdot x)=g \cdot x
$$

Logo, $h \in G_{g \cdot x}$

- $G_{g \cdot x} \subset g \cdot G_{x} \cdot g^{-1}$

Seja $h \in G_{g \cdot x}$, então $h(g \cdot x)=g \cdot x$. Tomando $l=g^{-1} h g$, temos

$$
l \cdot x=\left(g^{-1} h g\right) \cdot x=g^{-1} h(g \cdot x)=g^{-1} g \cdot x=x
$$

concluindo que $l \in G_{x}$. Como $h=g l g^{-1}$, resulta que $h \in g G_{x} g$.

Lema 2.9. Seja $(G, \cdot)$ um grupo de Lie. Definindo $\bar{G}:=(G, *)$, com $g_{1} * g_{2}=g_{2} \cdot g_{1}$, temos:

i) $\bar{G}$ é um grupo de Lie e os campos invariantes à esquerda de $\bar{G}$ são os campos invariantes à direita de $G$.

ii) A aplicação

$$
\begin{gathered}
i n v: G \longrightarrow \bar{G} \\
g \longmapsto g^{-1}
\end{gathered}
$$

é um isomorfismo de Lie.

iii) $\overline{[,]}=-[$,$] .$

\section{Demonstração:}

i) $\bar{G}$ tem a mesma estrutura suave de $G$, e $g_{1} * g_{2}=g_{2} \cdot g_{1}$ é suave, visto que $\cdot$ é suave. Além disso, $\bar{L}_{g}(x)=g * x=x \cdot g=R_{g}(x), \forall x \in G$, e com isso temos $d \bar{L}_{g}=d R_{g}, \forall g \in G$. Tomando $X$ um campo invariante à esquerda em $\bar{G}$, temos que $X=d \bar{L}_{g} X=d R_{g} X, \forall g \in G$ e portanto $X$ é um campo invariante à direita em $G$. Da mesma forma concluimos que todos os campos invariantes à direita em $G$ são campos invariantes à esquerda em $\bar{G}$.

ii) O fato da aplicação ser bijetora e suave segue imediatamente da hipótese de $G$ ser grupo de Lie, visto que o elemento inverso por $*$ é o mesmo que por $\cdot$. Para verificar que é homomorfismo de Lie, tome $g_{1}, g_{2} \in G$ :

$$
\left(g_{1} \cdot g_{2}\right)^{-1}=g_{2}^{-1} \cdot g_{1}^{-1}=g_{1}^{-1} * g_{2}^{-1}
$$

iii) Da proposição 1.45 , temos que $d i n v_{e}=-I$ é um isomorfismo de álgebras de Lie entre $T_{e} G$ e $T_{e} \bar{G} . \operatorname{Logo},-[X, Y]=\operatorname{dinv}_{e}[X, Y]=\overline{\left[\operatorname{dinv}_{e} X, d i n v_{e} Y\right]}=\overline{[-X,-Y]}=\overline{[X, Y]}$.

Proposição 2.10. Considere $\mu: G \times M \longrightarrow M$ uma ação à esquerda. 
i) Dado $\xi \in T_{e} G$, definimos $\vec{\xi} \in \Xi(M)$ como

$$
\vec{\xi}_{p}=\left.\frac{d}{d t}(\exp (t \xi) \cdot p)\right|_{t=0}
$$

ii) O fluxo de $\vec{\xi}$ é $\varphi_{t}(p):=\exp (t \xi) \cdot p$.

iii) A aplicação

$$
\begin{gathered}
\left(T_{e} G,-[,]\right) \underset{\xi}{\longrightarrow} \vec{\xi} \\
\exists(M)
\end{gathered}
$$

é homomorfismo de álgebra de Lie.

\section{Demonstração:}

i) Defina

$$
\begin{aligned}
s^{\xi}: M & \longrightarrow T_{e} G \times T M \\
p & \longmapsto\left(\xi, 0_{p}\right)
\end{aligned}
$$

assim,

$$
\begin{aligned}
d \mu_{(e, p)} s^{\xi}(p) & =d \mu_{(e, p)}\left(\xi, 0_{p}\right) \\
& =d \mu_{(e, p)}\left(\left.\frac{d}{d t}(\exp (t \xi), p)\right|_{t=0}\right) \\
& =\left.\frac{d}{d t} \mu(\exp (t \xi), p)\right|_{t-0} \\
& =\left.\frac{d}{d t} \exp (t \xi) \cdot p\right|_{t=0} \\
& =\overrightarrow{\xi_{p}}
\end{aligned}
$$

onde denotamos $\cdot=\mu($,$) .$

ii) Considere $\varphi_{t}(p)=\exp (t \xi) \cdot p$. Temos $\varphi_{0}(p)=e \cdot p=p$. Basta mostrar que:

$$
\left.\frac{d}{d t} \varphi_{t}(p)\right|_{t=t_{0}}=\left.\frac{d}{d t} \exp (t \xi) \cdot p\right|_{t=t_{0}}
$$

Façamos a mudança $s=t-t_{0}$, obtendo 


$$
\begin{aligned}
\left.\frac{d}{d t} \exp (t \xi) \cdot p\right|_{t=t_{0}} & =\left.\frac{d}{d s} \exp \left(\left(s+t_{0}\right) \xi\right) \cdot p\right|_{s=0} \\
& =\left.\frac{d}{d s}\left(\exp (s \xi) \exp \left(t_{0} \xi\right)\right) \cdot p\right|_{s=0} \\
& =\left.\frac{d}{d s} \exp (s \xi) \cdot\left(\exp \left(t_{0} \xi\right) \cdot p\right)\right|_{s=0} \\
& =\left.\frac{d}{d s} \exp (s \xi) \cdot \varphi_{t_{0}}(p)\right|_{s=0} \\
& =\vec{\xi}_{\varphi_{t_{0}}(p)}
\end{aligned}
$$

Portanto, $\varphi_{t}$ é o fluxo de $\vec{\xi}$.

iii) Dado $\xi \in T_{e} G$, definimos o campo $X^{\xi}(g, p)=\left(d R_{g} \xi, 0_{p}\right)$. Mostremos que os campos $\vec{\xi}$ e $\vec{X} \xi$ são $\mu$ relacionados.

$$
\begin{aligned}
d \mu_{(g, p)} X^{\xi}(g, p) & =d \mu_{(g, p)}\left(d R_{g} \xi, 0_{p}\right) \\
& =\left.d \mu_{(g, p)} \frac{d}{d t}(\exp (t \xi) g, p)\right|_{t=0} \\
& =\left.\frac{d}{d t} \mu(\exp (t \xi) g, p)\right|_{t=0} \\
& =\left.\frac{d}{d t}(\exp (t \xi) g) \cdot p\right|_{t=0} \\
& =\left.\frac{d}{d t} \exp (t \xi)(g \cdot p)\right|_{t=0} \\
& =\vec{\xi}_{g \cdot p} \\
& =\vec{\xi}_{\mu(g, p)}
\end{aligned}
$$

Resulta da proposição 1.41 que

$$
[\vec{\xi}, \vec{\eta}]_{g \cdot p}=d \mu_{(g, p)}\left[X^{\xi}, X^{\eta}\right]_{(g, p)}
$$

Usando a idéia sugerida pelo lema 2.9, podemos considerar $R(G)$ a álgebra dos campos invariantes à direita em $G$ e definir:

$$
\begin{aligned}
\theta: & R(G) \longrightarrow \Xi(G \times M) \\
& d R_{g} V_{e} \longmapsto\left(d R_{g} V_{e}, 0\right)
\end{aligned}
$$

A equação 2.1 implica que

$$
\begin{aligned}
d \mu \circ \theta: R(G) & \longrightarrow \Xi(M) \\
\xi & \longmapsto \vec{\xi}
\end{aligned}
$$


é um homomorfismo de álgebras de Lie. O resultado segue diretamente do isomorfismo provado no lema 2.9 .

Observação 2.11. Seja $\mu: G \times M \longrightarrow M$ uma ação à esquerda.

1. Podemos fixar $g \in G$ e definir:

$$
\begin{aligned}
\mu^{g}: M & \longrightarrow M \\
x & \longmapsto g \cdot x
\end{aligned}
$$

2. Analogamente, fixando $x \in M$ obtemos:

$$
\begin{aligned}
\mu_{x}: G & \longrightarrow M \\
g & \longmapsto g \cdot x
\end{aligned}
$$

Proposição 2.12. Seja $\mu: G \times M \longrightarrow M$ uma ação à esquerda. Então, para cada $x \in M$ fixado, temos $\operatorname{ker} d\left(\mu_{x}\right) g_{0}=T_{g_{0}}\left(g_{0} G_{x}\right)$.

Demonstração: Vamos demonstrar as inclusões:

i) $T_{g_{0}}\left(g_{0} G_{x}\right) \subset \operatorname{ker} d\left(\mu_{x}\right) g_{0}$

Seja $v \in T_{g_{0}}\left(g_{0} G_{x}\right)$. Então, existe $w \in T_{e}\left(G_{x}\right)$ de maneira que $d L_{g_{0}} w=v$. Pela regra da cadeia:

$$
\begin{aligned}
v & =d L_{g_{0}} w \\
& =\left.\frac{d}{d t} L_{g_{0}}(\exp (t w))\right|_{t=0} \\
& =\left.\frac{d}{d t} g_{0} \cdot(\exp (t w))\right|_{t=0}
\end{aligned}
$$

Apliquemos $d\left(\mu_{x}\right)_{g_{0}}$ a $v$ :

$$
\begin{aligned}
\left(d \mu_{x}\right)_{g_{0}} v & =\left.\left(d \mu_{x}\right)_{g_{0}} \frac{d}{d t} g_{0} \exp (t w)\right|_{t=0} \\
& =\left.\frac{d}{d t} \mu_{x}\left(g_{0} \exp (t w)\right)\right|_{t=0} \\
& =\left.\frac{d}{d t}\left(g_{0} \exp (t w)\right) \cdot x\right|_{t=0} \\
& =\left.\frac{d}{d t} g_{0} \cdot(\exp (t w) \cdot x)\right|_{t=0}
\end{aligned}
$$

Mas, como $w \in T_{e}\left(G_{x}\right)$, temos que $\exp (t w) \in G_{x}$ e portanto $\exp (t w) \cdot x=x$. Logo, a última expressão nos dá: 


$$
\left(d \mu_{x}\right)_{g_{0}} v=\left.\frac{d}{d t}\left(g_{0} \cdot x\right)\right|_{t=0}=0
$$

Com isto provamos que $v \in \operatorname{ker} d\left(\mu_{x}\right)_{g_{0}}$

ii) $\operatorname{ker} d\left(\mu_{x}\right) g_{0} \subset T_{g_{0}}\left(g_{0} G_{x}\right)$

Se $v \in \operatorname{ker} d\left(\mu_{x}\right) g_{0} \subset T_{g_{0}} G$, então existe $w \in T_{e} G \operatorname{com} d L_{g_{0}} w=v$. Novamente, pela regra da cadeia:

$$
\begin{aligned}
v & =d L_{g_{0}} w \\
& =\left.\frac{d}{d t} L_{g_{0}}(\exp (t w))\right|_{t=0} \\
& =\left.\frac{d}{d t} g_{0} \exp (t w)\right|_{t=0}
\end{aligned}
$$

Usando a hipótese,

$$
\begin{aligned}
d\left(\mu_{x}\right)_{g_{0}} v=0 & \left.\Rightarrow d\left(\mu_{x}\right)_{g_{0}} \frac{d}{d t} g_{0} \exp (t w)\right|_{t=0}=0 \\
& \left.\Rightarrow \frac{d}{d t} \mu_{x}\left(g_{0} \exp (t w)\right)\right|_{t=0}=0 \\
& \left.\Rightarrow \frac{d}{d t} \mu\left(g_{0} \exp (t w), x\right)\right|_{t=0}=0 \\
& \left.\Rightarrow \frac{d}{d t} \mu\left(g_{0}, \exp (t w) \cdot x\right)\right|_{t=0}=0
\end{aligned}
$$

Note que $g_{0}$ está fixado, e com notação da observação 2.11 temos:

$$
\begin{array}{r}
\left.\frac{d}{d t} \mu^{g_{0}}(\exp (t w) \cdot x)\right|_{t=0}=0 \\
\left.\Rightarrow d \mu^{g_{0}} \frac{d}{d t}(\exp (t w) \cdot x)\right|_{t=0}=0
\end{array}
$$

Pelo item $i)$ da proposição $2.10,\left.\frac{d}{d t}(\exp (t w) \cdot x)\right|_{t=0}=\vec{w}_{x}$ campo em $M$, e nossos cálculos indicam $d \mu^{g_{0}} \vec{w}_{x}=0$. Como $d \mu^{g_{0}}$ é invertível, resulta $\vec{w}_{x}=0$. Usando o item $i i$ ) da proposição 2.10 , temos o fluxo de $\vec{w}$ dado por $\varphi_{t}(x)=\exp (t w) \cdot x$, de onde concluimos por unicidade de $\operatorname{EDO}$ que $\exp (t w) \cdot x=x$ para todo $t$ e portanto $\exp (t w)$ é curva em $G_{x}$. Lembre que $v=\left.\frac{d}{d t} g_{0} \exp (t w)\right|_{t=0}$ e pelo último argumento $g_{0} \exp (t w)$ é curva em $g_{0} G_{x}$, sendo possível concluir que $v \in T_{g_{0}}\left(g_{0} G_{x}\right)$.

Definição 2.13. Aplicações $\varphi: M \longrightarrow N$ são próprias se $\varphi^{-1}(K) \subset M$ for compacto, para todo 
compacto $K \subset N$.

Definição 2.14 (Ações Próprias). A ação à esquerda $\mu: G \times M \longrightarrow M$ é própria se a aplicação

$$
\begin{aligned}
H: G \times M & \longrightarrow M \times M \\
(g, x) & \longmapsto(g \cdot x, x)
\end{aligned}
$$

for própria.

Proposição 2.15. São equivalentes:

i) $G \times M \longrightarrow M$ é uma ação própria

ii) Sejam $L, K \subset M$ compactos, então $\{g \in G: g \cdot K \cap L \neq \emptyset\}$ é compacto

iii) Sejam $\left(x_{n}\right)$ e $\left(g_{n} x_{n}\right)$ sequências em $M$ tais que $x_{n} \longrightarrow x \in M$ e $g_{n} x_{n} \longrightarrow y \in M$. Então, $\left(g_{n}\right)$ admite uma subsequência convergente em $G$.

\section{Demonstração:}

- $i) \Rightarrow i i)$. Seja $B=\{g \in G: g \cdot K \cap L \neq \emptyset\}$ e

$$
\begin{gathered}
H: G \times M \longrightarrow M \times M \\
(g, x) \longmapsto(g \cdot x, x)
\end{gathered}
$$

então, $W=H^{-1}(L, K)=\{(g, x) \in G \times M: g \cdot x \in L$ e $x \in K\}$ é compacto. Como a projeção $\pi_{1}: G \times M \longrightarrow G$ é contínua, $\pi_{1}(W)$ é também compacto. Mostremos que $\pi_{1}(W)=B$ :

$\pi_{1}(W) \subset B$, pois se $g \in \pi_{1}(W) \Rightarrow(g, x) \in W \Rightarrow(g \cdot x, x) \in L \times K \Rightarrow g K \cap L \neq \emptyset \Rightarrow g \in B$

$B \subset \pi_{1}(W)$, pois se $g \in B \Rightarrow g K \cap L \neq \emptyset \Rightarrow \exists x \in K: g \cdot x \in L \Rightarrow(g, x) \in W \Rightarrow g \in \pi_{1}(W)$

- $i i) \Rightarrow i$ ). Tomando $A \subset M \times M$ compacto, podemos encontrar $L, K$ compactos em $M$ de maneira que $A \subset L \times K$. Por $i i), B=\{g \in G: g \cdot K \cap L \neq \emptyset\}$ é compacto e $H^{-1}(A) \subset H^{-1}(L, K) \subset(B, K)$. Visto que $H^{-1}(A)$ é fechado e está contido em um compacto, segue que $H^{-1}(A)$ é também compacto.

- $i i) \Rightarrow$ iii). Seja $K=\left\{x_{n}\right\} \cup\{x\}$ e $L=\left\{g_{n} \cdot x_{n}\right\} \cup\{y\}$. K e $L$ são compactos e para todo $n$, $g_{n} \in B=\{g \in G: g \cdot K \cap L \neq \emptyset\}$. Como assumimos $B$ compacto, $\left(g_{n}\right)$ admite uma subsequência convergente.

- iii) $\Rightarrow$ ii). Tomando $L$ e $K$ compactos em $M$, vamos considerar $B=\{g \in G: g \cdot K \cap L \neq \emptyset\}$ e uma sequência $\left(g_{n}\right)$ em $B$. Então, existe $\left(x_{n}\right)$ em $K$ tal que $\left(g_{n} \cdot x_{n}\right)$ é sequência em $L$. Como $K$ e $L$ são compactos, podemos supor (tomando uma subsequência se necessário) que $x_{n} \longrightarrow x \in K$ e $g_{n} \cdot x_{n} \longrightarrow y \in L$. De $\left.i i i\right),\left(g_{n}\right)$ admite uma subsequência convergente e portanto $B$ é compacto.

Observação 2.16. Se a ação for própria, decorre imediatamente da definição que $G_{x}$ é compacto, pois $H^{-1}(x, x)=\left\{\left(G_{x}, x\right)\right\}$ 


\section{Exemplo 2.17. .}

1. Se $G$ é compacto, então $G \times M \longrightarrow M$ é própria.

2. Se $H \subset G$ um subgrupo de Lie fechado, então

$$
\begin{gathered}
G \times H \longrightarrow G \\
(g, h) \longmapsto g h
\end{gathered}
$$

é própria.

\subsection{Fibrados}

Definição 2.18 (Fibrado Coordenado). Sejam $E, F, B$ variedades suaves e $G$ um grupo de Lie. Suponha que $G \times F \longrightarrow F$ seja uma ação à esquerda efetiva, e $\pi: E \longrightarrow B$ uma aplicação suave sobrejetora. Suponha ainda que $B$ admite uma cobertura aberta $\left\{\mathcal{U}_{\alpha}\right\}$ e existem difeomorfismos $\psi_{\alpha}: \mathcal{U}_{\alpha} \times F \longrightarrow \pi^{-1}\left(\mathcal{U}_{\alpha}\right)$ satisfazendo às propriedades abaixo:

a) $\pi \circ \psi_{\alpha}=\pi_{1}$, onde $\pi_{1}(b, f)=b$

b) Sejam $\mathcal{U}_{\alpha}, \mathcal{U}_{\beta}$ abertos tais que $\mathcal{U}_{\alpha} \cap \mathcal{U}_{\beta} \neq \emptyset$. Então, $\psi_{\beta}^{-1} \psi_{\alpha}(b, f)=\left(b, \theta_{\alpha \beta}(b) f\right)$, onde $\theta_{\alpha \beta}(b) \in G$ e $\theta_{\alpha \beta}: \mathcal{U}_{\alpha} \cap \mathcal{U}_{\beta} \longrightarrow G$ é suave.

A 7-upla $\left(E, B, F, G, \pi, \mathcal{U}_{\alpha}, \psi_{\alpha}\right)$ é o fibrado coordenado.

Observação 2.19. Na definição acima, os elementos que copõem um fibrado recebem algumas denominações especiais, sendo elas:

E: Espaço Total

B: Base

F: Fibra

G: Grupo Estrutural

$\pi$ : Projeção

$\psi_{\alpha}$ : Trivialização Local

$\theta_{\alpha \beta}$ : Função de Transição

Definição 2.20. Dois fibrados coordenados $\left(E, B, F, G, \pi, \mathcal{U}_{\alpha}, \psi_{\alpha}\right) \mathrm{e}$ $\left(E, B, F, G, \pi, \mathcal{V}_{\beta}, \varphi_{\beta}\right)$ são equivalentes se $\varphi_{\beta}^{-1} \circ \psi_{\alpha}(b, f)=\left(b, \tilde{\theta}_{\beta \alpha}(b) f\right)$. Assim, a classe de equivalência é chamada simplesmente de fibrado.

Exemplo 2.21. Alguns exemplos de fibrados são: 
1. Seja $C:=\left\{(x, y, z) \in \mathbb{R}^{3}: x^{2}+y^{2}=1\right\}$ o cilindro circular reto. Para ilustrar a definição de fibrado, basta tomar $E=C, B=S^{1}, F=\mathbb{R}$ e $G=\{I\}$. Geometricamente falando, as fibras são as retas verticais e a base o círculo formado pelo cilindro na intersecção com o plano $x O y .\left\{\mathcal{U}_{\alpha}\right\}$ são as vizinhanças parametrizadas de $S^{1}, \pi$ leva cada reta vertical $r$ em $r \cap S^{1}$ e $\theta_{\alpha \beta}(b)=1, \forall b \in B$.

2. Considerando $E$ a faixa de Möbius, $B=S^{1}, F=\mathbb{R}$ e $G=\{I,-I\} \cong \mathbb{Z}_{2}$, temos as vizinhanças $\mathcal{U}_{\alpha}=\left\{e^{i \theta} \in S^{1}:-\pi-\frac{\pi}{3}<\theta<\frac{\pi}{3}\right\}$ e $\mathcal{U}_{\beta}=\left\{e^{i \theta} \in S^{1}:-\frac{\pi}{3}<\theta<\pi+\frac{\pi}{3}\right\}$. Assim, $\mathcal{U}_{\alpha} \cap \mathcal{U}_{\beta}=\mathcal{W}_{1} \cap \mathcal{W}_{2}$ onde $\left.\theta_{\beta \alpha}\right|_{\mathcal{W}_{1}}=I$ e $\left.\theta_{\beta \alpha}\right|_{\mathcal{W}_{2}}=-I$.

3. (Fibrado Tangente)

Dada uma variedade $M$, podemos considerar $E=T M, F=\mathbb{R}^{n}, B=M$, sendo $\pi$ a projeção canônica de $T M$ em $M$. Daí,

$$
\psi_{\beta}^{-1} \psi_{\alpha}(b, v)=\left(b, d\left(\varphi_{\beta}^{-1} \varphi_{\alpha}\right)_{b} v\right)
$$

onde $\left\{\varphi_{\alpha}\right\}$ é atlas de $M$. Neste caso, temos $G=G L_{n}(\mathbb{R})$.

Definição 2.22 (Fibrado Vetorial e Fibrado Principal). .

a) Um fibrado $(E, B, F, G, \pi)$ é chamado fibrado vetorial se $F=\mathbb{R}^{n}$ e $G \subset G L_{n}(\mathbb{R})$.

b) Um fibrado $(E, B, F, G, \pi)$ é chamado um fibrado principal se $F=G$. Neste caso, denotamos apenas $(E, B, G, \pi)$.

Proposição 2.23. Seja $(E, B, G, \pi)$ um fibrado principal. Então, existe uma ação livre e própria à direita de $G$ em $E$ tal que $\left\{\pi^{-1}(x)\right\}_{x \in B}$ são órbitas.

Demonstração: Sendo $: G \times G \longrightarrow G$ a ação dada pela definição de fibrado, vamos definir uma ação à direita $*: E \times G \longrightarrow G$ por $x * g:=\psi_{\alpha}(b, f \cdot g)$, onde $x=\psi_{\alpha}(b, f)$. Mostremos que nos pontos $b \in \mathcal{U}_{\alpha} \cap \mathcal{U}_{\beta}$ a ação independe da trivialização.

Do item b) da definição 2.18 , se $b \in \mathcal{U}_{\alpha} \cap \mathcal{U}_{\beta}$ temos que $x=\psi_{\alpha}(b, f)=\psi_{\beta}\left(b, \theta_{\alpha \beta}(b) \cdot f\right)$. Daí,

$$
\begin{aligned}
\psi_{\alpha}(b, f \cdot g) & =\psi_{\beta}\left(b, \theta_{\alpha \beta}(b) \cdot(f \cdot g)\right) \\
& =\psi_{\beta}\left(b,\left(\theta_{\alpha \beta}(b) \cdot f\right) \cdot g\right)
\end{aligned}
$$

Segue diretamente da definição de ação e de fibrado coordenado que a ação é livre e $\pi^{-1}(b)$ é órbita, para todo $b \in B$. Mostremos que a ação é própria: se $\left(b_{n}, f_{n}\right) \longrightarrow(b, f)$ e $\left(b_{n}, f_{n} \cdot g_{n}\right) \longrightarrow(b, k)$, então $f_{n} \cdot g_{n} \longrightarrow k$ e, como $f_{n} \longrightarrow f$, temos que $g_{n} \longrightarrow f^{-1} \cdot k \in G$. Compondo por trivialização concluimos a prova. 
Definição 2.24 (Espaço de Órbita). Dada uma ação à esquerda $\mu: G \times M \longrightarrow M$, considere a relação de equivalência

$$
x \sim y \Leftrightarrow \exists g \in G: g \cdot x=y
$$

Definimos o espaço de órbita de $\mu$ por:

$$
M / G=\{[x]: x \in M\}
$$

Observação 2.25. .

1. O espaço $M / G$ pode ser dotado da topologia quociente, isto é, $\mathcal{U}$ é aberto em $M / G$ se $\pi^{-1}(\mathcal{U})$ é aberto em $M$.

2. $\pi: M \longrightarrow M / G$ é contínua e aberta.

$\pi$ é contínua, pela definição de topologia quociente. Agora, tomando $\mathcal{U}$ aberto, temos que $\pi^{-1}(\pi(\mathcal{U}))=\cup_{g \in G} g \mathcal{U}$, o qual é união de abertos.

3. $\pi: M \longrightarrow M / G$ não é fechada.

Como exemplo, podemos tomar $M=\mathbb{R}^{2}$ e $G=\left\{f_{\alpha}(x, y)=(x, y+\alpha)\right\}$. O conjunto $C=$ $\left\{(x, y) \in \mathbb{R}^{2}: x y=1\right\}$ é fechado, porém $\pi(C)=\mathbb{R}-\{0\}$ não é fechado.

4. $M / G$ pode ser não-Hausdorff. Como contra-exemplo, temos a ação irracional do toro.

Proposição 2.26. Se $G \times M \longrightarrow M$ for própria, então $M / G$ será Hausdorff.

Demonstração: A prova se seguirá por meio das afirmações:

Afirmação 2.27. Um espaço topológico $N$ é Hausdorff se, e somente se, $\Delta:=\{(x, x) \in N \times N\}$ é fechado em $N \times N$

Afirmação 2.28. Como a ação é própria, o conjunto

$$
C=\{(x, g \cdot x) \in M \times M: x \in M, g \in G\}
$$

é fechado.

Prova: De fato, se $x_{n} \longrightarrow x$ e $g_{n} \cdot x_{n} \longrightarrow y$, então existe subsequência (usando o mesmo índice $n$ ) $g_{n} \longrightarrow g$ o que resulta em $g \cdot x=y$ (usamos aqui o fato de $M$ ser Hausdorff). Assim temos que $(x, y) \in C$ e portanto $C$ é fechado.

Afirmação 2.29 .

$$
\begin{aligned}
\tilde{\pi}: M \times M & \longrightarrow \frac{M}{G} \times \frac{M}{G} \\
(x, y) & \longrightarrow(\pi(x), \pi(y))
\end{aligned}
$$


é contínua, aberta e sobrejetora.

Prova: Isto segue diretamente dos itens 1) e 2) da observação 2.25.

Afirmação 2.30. $\tilde{\pi}(C)=\Delta$, diagonal de $\frac{M}{G} \times \frac{M}{G}$, e $\tilde{\pi}^{-1}(\Delta)=C$

Prova: Consequência direta da definição de $C$.

Como $\tilde{\pi}$ é aberta, $\tilde{\pi}\left(C^{c}\right)$ é aberto e, como $\tilde{\pi}$ é sobrejetora, resulta que $\Delta$ é fechado. O resultado segue então da afirmação 2.27

Teorema 2.31. Seja $\mu: M \times G \longrightarrow M$ uma ação à direita livre e própria. Então $M / G$ admite uma estrutura de variedade tal que $(M, \pi, M / G, G)$ é fibrado principal, sendo $\pi: M \longrightarrow M / G$.

Demonstração: Já sabemos pela proposição 2.26 que $M / G$ é espaço de Hausdorff. Seja $x \in M$ e $S_{x}$ uma subvariedade de $M$ tal que

$$
T_{x} M=T_{x} S_{x} \oplus d\left(\mu_{x}\right)_{e} \mathfrak{g}
$$

Vamos provar por meio de lemas:

Lema 2.32. Reduzindo $S_{x}$ se necessário, temos que a restrição $\varphi:=\left.\mu\right|_{S_{x} \times G}$ é um difeomorfismo.

Prova: Pela proposição 2.12,

$$
\text { ker } d\left(\mu_{x}\right) g_{0}=T_{g_{0}}\left(g_{0} G_{x}\right)
$$

Visto que a ação é livre $\left(G_{x}=\{e\}, \forall x \in M\right)$, temos

$$
\operatorname{ker} d\left(\mu_{x}\right)_{e}=\{0\}
$$

e portanto $d\left(\mu_{x}\right)_{e}$ é injetora. Como $d \mu$ é contínua, podemos reduzir $S_{x}$ se necessário, e obter:

$$
\begin{gathered}
d\left(\mu_{y}\right)_{e}: \mathfrak{g} \longrightarrow T_{y} M \text { injetora para todo } y \in S_{x} \\
T_{y} M=T_{y} S_{x} \oplus d\left(\mu_{y}\right)_{e} \mathfrak{g}
\end{gathered}
$$

Por outro lado

$$
\mu^{e}: S_{x} \longrightarrow M
$$

tem por derivada $d \mu^{e}=I$, e então

$$
\left.\mu\right|_{S_{x} \times\{e\}}: S_{x} \times\{e\} \longrightarrow M \text { é imersão }
$$


Das equações 2.2, 2.3 e 2.4 resulta que $d \mu_{(y, e)}$ é isomorfismo para todo $y \in S_{x}$.

Vamos provar agora que o mesmo ocorre nos demais pontos $g \in G$. Para isso, consideramos os caminhos suaves $\alpha$ em $S_{x}$ e $\beta$ em $G \operatorname{com} \alpha(0)=y$ e $\beta(0)=e$.

$$
\begin{aligned}
d \mu_{(y, g)}\left(\alpha^{\prime}(0), d R_{g} \beta^{\prime}(0)\right) & =\left.\frac{d}{d t} \mu(\alpha(t), \beta(t) \cdot g)\right|_{t=0} \\
& =\left.\frac{d}{d t} \mu(\mu(\alpha(t), \beta(t)), g)\right|_{t=0} \\
& =d \mu_{y}^{g} d \mu_{(y, e)}\left(\alpha^{\prime}(0), \beta^{\prime}(0)\right)
\end{aligned}
$$

Como $d \mu_{y}^{g}$ é inversível, segue da igualdade acima que $d \mu_{(y, g)}$ é isomorfismo. Aplicando o teorema da função inversa, concluimos que $\varphi:=\left.\mu\right|_{S_{x} \times G}$ é difeomorfismo local.

Vamos mostrar que $\varphi$ é injetora para concluir que é um difeomorfismo global. Suponha por absurdo que não seja injetora, então podemos tomar duas sequências $\left(x_{n}, g_{n}\right)$ e $\left(y_{n}, h_{n}\right)$ em $S_{x} \times G$ com

$$
\begin{aligned}
& x_{n} \longrightarrow x \\
& y_{n} \longrightarrow x
\end{aligned}
$$

de maneira que $x_{n} \cdot g_{n}=y_{n} \cdot h_{n}$ mas $\left(x_{n}, g_{n}\right) \neq\left(y_{n}, h_{n}\right)$.

Tomando

$$
k_{n}=g_{n} h_{n}^{-1}
$$

temos

$$
x_{n} \cdot k_{n}=y_{n}
$$

e daí

$$
x_{n} \cdot k_{n} \longrightarrow x
$$

Pela hipótese da ação ser própria, é possível encontrar uma subsequência convergente $k_{n} \longrightarrow k$ (mantemos o mesmo índice por simplicidade de notação). Assim, temos também

$$
x_{n} \cdot k_{n} \longrightarrow x \cdot k
$$

Das equações 2.6 e 2.7 , resulta

$$
x \cdot k=x
$$

e portanto $k \in G_{x}=\{e\}$. Pela equação 2.5

$$
g_{n} \cdot h_{n}^{-1} \longrightarrow e
$$

A equação 2.8 juntamente com nossa suposição por absurdo contraria o fato de $\varphi$ ser injetora na 
vizinhança de $(x, e)$. Este argumento prova que $\varphi$ é um difeomorfismo global.

Lema 2.33. $\pi\left(S_{x}\right)$ é aberto em $M / G$.

Prova: De fato, $\pi^{-1}\left(\pi\left(S_{x}\right)\right)=\left(S_{x}\right) G=\left\{s \cdot g: s \in S_{x}, g \in G\right\}$, o qual é aberto. Segue do lema 2.32 que cada órbita em $\left(S_{x}\right) G$ intersecta $S_{x}$ em um único ponto, e portanto $\left.\pi\right|_{S_{x}}: S_{x} \longrightarrow \pi\left(S_{x}\right)$ é homeomorfismo.

Desejamos mostrar que:

- Para outra variedade $S_{y}$, temos

$$
\left.\left(\left.\pi\right|_{S_{y}}\right)^{-1} \circ \pi\right|_{S_{x}}: \mathcal{U} \longrightarrow \mathcal{V} \text { é suave. }
$$

(no lema 2.34, especificamos $\mathcal{U}$ e $\mathcal{V}$ )

- $\psi_{y}^{-1} \circ \psi_{x}(b, g)=\left(b, \theta_{y x}(b) g\right)$, onde

$$
\begin{aligned}
\psi_{x}: \pi\left(S_{x}\right) \times G & \longrightarrow \pi^{-1}\left(S_{x}\right) \\
(b, g) & \longmapsto\left(\left.\pi\right|_{S_{x}}\right)^{-1}(b) \cdot g
\end{aligned}
$$

Mostraremos estes dois itens no próximo lema:

Lema 2.34. Sendo $x, y \in M$ e $S_{x}, S_{y}$ tomados como no início desta demonstração, suponha que

$$
\emptyset \neq \mathcal{W}=\left(S_{x}\right) G \cap\left(S_{y}\right) G
$$

Então:

i) Exitem abertos $\mathcal{U}$ em $S_{x}$ e $\mathcal{V}$ em $S_{y}$ tal que

$$
\mathcal{W}=\varphi(\mathcal{U}, G)=\varphi(\mathcal{V}, G)
$$

ii) A aplicação $K: \mathcal{V} \longrightarrow \mathcal{U}$ definida por $K(z)=(z) G \cap S_{x}$ é suave. 
Prova:

i) Pelo lema 2.32, $\varphi^{-1}(\mathcal{W})$ é aberto em $S_{x} \times G$ e invariante pela ação de $G$. Portanto, é da forma $\mathcal{U} \times G$. Pelo mesmo argumento, é também da forma $\mathcal{V} \times G$.

ii) Temos que $\varphi^{-1}(\mathcal{U})=\{(z, \tilde{\theta}(z)): z \in \mathcal{V}\}$. Logo $\tilde{\theta}$ é suave, e consequentemente $K(z)=z \cdot \tilde{\theta}(z)$ é suave e $K(\mathcal{V})=\mathcal{U}$. Agora, basta definir

$$
\theta(b):=\tilde{\theta}\left(\left(\pi||_{\mathcal{V}}\right)^{-1}(b)\right)
$$

Corolário 2.35. Seja $G$ um grupo de Lie e $H$ um subgrupo normal. Então, $G / H$ admite estrutura suave que o torna um grupo de Lie. Mais ainda, $\pi: G \longrightarrow G / H$ é homomorfismo de Lie.

Demonstração: Pelo exemplo 2.4.1, a ação $(g, h) \longrightarrow g \cdot h$ é livre e própria. Do teorema 2.31, $G / H$ é variedade e $\pi: G \longrightarrow G / H$ é suave. Tomando

$$
\alpha(a, b)=a \cdot b^{-1}
$$

temos do fato de $H$ ser subgrupo normal que o diagrama

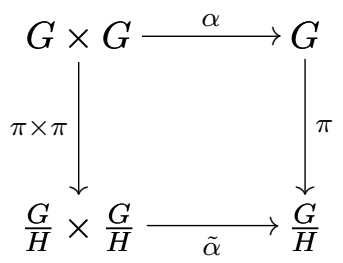

comuta e portanto

$$
\tilde{\alpha}(a H, b H)=a b^{-1} H
$$

A comutatividade do diagrama e o fato de $\pi$ admitir seções suaves (isto é, existe $s: \mathcal{U} \subset G / H \longrightarrow G$ tal que $\pi \circ s(b)=b$ ) garantem que $\tilde{\alpha}$ é suave.

Proposição 2.36. Seja $\mu: G \times M \longrightarrow M$ uma ação $e$, fixando $x \in M$, considere $\mu_{x}$ conforme a observação 2.11. Então:

i) $\tilde{\mu_{x}}: G / G_{x} \rightarrow M$ tal que $\tilde{\mu_{x}} \circ \pi=\mu_{x}$ é imersão injetora.

ii) Se a ação for própria, $\tilde{\mu_{x}}: G / G_{x} \rightarrow M$ é um mergulho. Em particular, $G(x)$ é variedade mergulhada. 


\section{Demonstração:}

i) Segue do teorema 2.31 que $G / G_{x}$ é variedade. Visto que $g \cdot G_{x}=\pi^{-1}([g])$ e $\mu_{x}\left(g \cdot G_{x}\right)=g \cdot x$, concluimos que existe uma aplicação $\tilde{\mu}_{x}$ que faz com que o diagrama

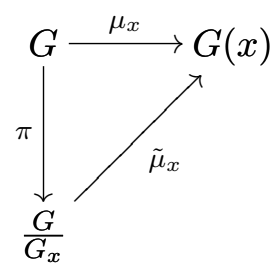

comute, ou seja, $\mu_{x}=\tilde{\mu_{x}} \circ \pi$. Como $\pi: G \longrightarrow G_{x}$ admite seções locais, resulta que $\tilde{\mu}_{x}$ é suave. Da proposição 2.12, $d \tilde{\mu}_{x}$ é injetora. Logo, $\tilde{\mu}_{x}: \frac{G}{G_{x}} \longrightarrow G(x)$ é bijeção, em particular sem auto-interseção.

ii) Desejamos mostrar que existe vizinhança $\mathcal{W}$ de $g_{0} \cdot x$ em $M$ e vizinhança $\mathcal{U}$ de $g_{0} \cdot G_{x}$ em $\frac{G}{G_{x}}$ tal que

$$
\tilde{\mu}_{x}(\mathcal{U})=\mathcal{W} \cap G(x)
$$

Suponha que isto não seja verdade. Então, existe sequência $\left(g_{n} x\right)$ tal que

$$
g_{n} \cdot x \longrightarrow g_{0} \cdot x
$$

e

$$
\pi\left(g_{n}\right) \nrightarrow \pi\left(g_{0}\right)
$$

Visto que a ação é própria, existe uma subsequência (a qual usamos o mesmo índice) $g_{n}$ convergente a $\tilde{g}$. Pela equação 2.9

$$
\tilde{g} \cdot x=g_{0} \cdot x
$$

e portanto $\pi(\tilde{g})=\pi\left(g_{0}\right)$, uma contradição.

Sabemos pelo teorema 1.47 que dado um grupo de Lie conexo $G$, existe um único grupo de Lie conexo e simplesmente conexo $\tilde{G}$ e uma aplicação recobrimento $\rho: \tilde{G} \longrightarrow G$.

Proposição 2.37. Seja $G$ um grupo de Lie conexo e considere $\rho: \tilde{G} \longrightarrow G$ dada acima. Então:

i) $H=\rho^{-1}(e)$ é um subgrupo de Lie fechado, normal e discreto. Além disso, $\mathrm{ghg}^{-1}=h$, para todo $h \in H, g \in \tilde{G}$.

ii) Gé isomorfo a $\tilde{G} / H$

iii) $\pi_{1}(G)$ é abeliano.

\section{Demonstração:}


i) Como $\rho$ é contínuo, evidentemente $H$ é fechado e, sendo $\rho$ um homomorfismo, podemos tomar $g \in \tilde{G}$ arbitrário e obter:

$$
\begin{aligned}
\rho\left(g H g^{-1}\right) & =\rho(g) \rho(H) \rho\left(g^{-1}\right) \\
& =\rho(g) e \rho\left(g^{-1}\right) \\
& =e
\end{aligned}
$$

Portanto $H$ é normal. Que $H$ é discreto segue diretamente da definição de recobrimento.

Fixando $h \in H$, considere a função

$$
\begin{aligned}
f: \tilde{G} & \longrightarrow H \\
g & \longmapsto g h g^{-1}
\end{aligned}
$$

Como $\{h\}$ é aberto e fechado em $H$ e a função é contínua, segue que $f^{-1}(h)$ é aberto e fechado em $\tilde{G}$. Mas $\tilde{G}$ é conexo, e portanto

$$
f^{-1}(h)=\tilde{G}
$$

Logo, $g h g^{-1}=h$ para todo $g \in \tilde{G}$.

ii) Visto que

$$
\rho(a h)=\rho(a) \text { para todo } a \in G, h \in H
$$

temos

$$
\rho \circ \pi^{-1}(a H)=\rho(a)
$$

e portanto fica bem definida

$$
\varphi:=\rho \circ \pi^{-1}: \tilde{G} / H \longrightarrow G
$$

Note que $\varphi$ é homomorfismo contínuo, e por consequência um homomorfismo de Lie (vide corolário 1.71). Como $\varphi$ é bijetora, é um isomorfismo.

Como $\pi: \tilde{G} \longrightarrow \tilde{G} / H$ é fibrado (teorema 2.31), segue que $\varphi$ é suave e, dado que $H$ é discreto, $d \pi$ e $d \rho$ são isomorfismos, resultando $d \varphi$ um isomorfismo. Pelo teorema da função inversa, $\varphi$ é um difeomorfismo local e, como é bijetor, é isomorfismo de Lie.

iii) Sejam $\alpha_{i}$ caminhos em $G \operatorname{com} \alpha_{i}(0)=e=\alpha_{i}(1)$, para $i=1,2$ e $\tilde{\alpha}_{i}$ levantamento de $\alpha_{i}$ (isto é, $\left.\rho\left(\tilde{\alpha}_{i}\right)=\alpha_{i}\right) \operatorname{com} \tilde{\alpha_{i}}(0)=\tilde{e}$.

Defina

$$
h_{i}:=\tilde{\alpha}_{i}(1)
$$

Note que: 
(1) $\beta=\tilde{\alpha}_{2} \cdot h_{1}$ é o levantamento de $\alpha_{2}$ que passa por $h_{1}$, isto é, $\beta(0)=h_{1}$ e $\rho(\beta)=\alpha_{2}$.

(2) $\beta(1)=h_{2} \cdot h_{1}$

(1) e (2) implicam que:

$$
\begin{aligned}
\widetilde{\alpha_{2} * \alpha_{1}}(1) & =h_{2} \cdot h_{1} \\
& =h_{1} \cdot h_{2} \\
& =\widetilde{\alpha_{1} * \alpha_{2}}(1)
\end{aligned}
$$

onde * é concatenação.

Assim, $\widetilde{\alpha_{2} * \alpha_{1}}$ e $\widetilde{\alpha_{1} * \alpha_{2}}$ são curvas em $\tilde{G}$ com mesmos pontos finais e iniciais, e portanto são homotópicas por homotopia que fixa os extremos. Isto implica que $\alpha_{2} * \alpha_{1}$ e $\alpha_{1} * \alpha_{2}$ são homotópicas por homotopia que fixa $e$, ou seja:

$$
\begin{aligned}
{\left[\alpha_{2}\right] *\left[\alpha_{1}\right] } & =\left[\alpha_{2} * \alpha_{1}\right] \\
& =\left[\alpha_{1} * \alpha_{2}\right] \\
& =\left[\alpha_{1}\right] *\left[\alpha_{2}\right]
\end{aligned}
$$

Concluimos que $\pi_{1}(G)$ é abeliano.

\subsection{Existência de Slice}

Iniciamos esta seção definindo subvariedade slice em $\mathrm{x}$, exibindo exemplos do que isso significa e terminamos por provar que se a ação for própria é sempre possível encontrar uma subvariedade desse tipo.

Definição 2.38 (Slice). Seja $\mu: G \times M \longrightarrow M$ uma ação à esquerda e $x \in M$ fixado. Dizemos que uma subvariedade $S_{x}$ contendo $x$ é slice em $x$ se:

a)

$$
T_{x} M=T_{x} S_{x} \oplus T_{x} G(x)
$$

e

$$
T_{y} M=T_{y} S_{x}+T_{y} G(y)
$$

para todo $y \in S_{x}$.

b) $S_{x}$ é invariante pela ação de $G$, ou seja, se $y \in S_{x}$ e $g \in G_{x}$, então $g \cdot y \in S_{x}$

c) Se $y \in S_{x}, g \in G$ e $g \cdot y \in S_{x}$, então $g \in G_{x}$ 
Lema 2.39. Seja $\mu: G \times M \longrightarrow M$ uma ação e $x \in M$ fixado. Suponha a existência de uma subvariedade $S$ tal que $T_{x} M=T_{x} G(x) \oplus T_{x} S$. Então:

i) Existe uma subvariedade $C \subset G$ contendo e tal que

$$
\mathfrak{g}=\mathfrak{g}_{x} \oplus T_{x} C
$$

ii) Reduzindo $S$, se necessário, temos que $\varphi:=\left.\mu\right|_{C \times S}$ é um difeomorfismo.

Demonstração: Denotando $\mathfrak{g}_{x}:=T_{e}\left(G_{x}\right)$, seja $V$ tal que

$$
\mathfrak{g}=\mathfrak{g}_{x} \oplus V
$$

Considere $\mathcal{B}_{\varepsilon}(0) \subset V$, com $\varepsilon>0$ suficientemente pequeno, e defina

$$
C:=\exp \left(\mathcal{B}_{\varepsilon}(0)\right)
$$

Pela proposição $2.12, \operatorname{ker}\left(d \mu_{x}\right)_{e}=T_{e} G_{x}$, de onde concluimos que

(1) $\left.d\left(\mu_{x}\right)_{e}\right|_{V}$ é injetor

(2) $d\left(\mu_{x}\right)_{e} V=T_{x} G(x)$.

Por outro lado,

(3) $\left.d \mu^{e}\right|_{T_{x} S}=I$

(1), (2) e (3) acima implicam que $(d \varphi)_{(e, x)}$ é um isomorfismo, e o resultado segue do teorema da função inversa.

Lema 2.40. Seja $\mu: G \times M \longrightarrow M$ uma ação própria e $G_{x}$ o grupo de isotropia de um elemento $x \in M$ fixado. Então, existe uma métrica riemanniana em $M$ tal que $G_{x}$ é subgrupo do grupo de isometrias de $M$. Em particular:

i) $\exp _{x}: \mathcal{B}_{\varepsilon}(0) \longrightarrow \mathcal{U}$ é difeomorfismo

ii) $d\left(\exp _{x}\right)_{0}=I$

iii) $\mu^{g}\left(\exp _{x}(v)\right)=\exp _{x}\left(d \mu^{g} v\right)$ sendo $g \in G_{x}$.

Demonstração: Tomando uma métrica arbitrária ( , ) em $M$, definimos:

$$
\langle x, y\rangle_{p}=\int_{G_{x}}\left(d \mu^{g} x, d \mu^{g} y\right)_{g \cdot p} w
$$


onde $w$ é uma $n$-forma volume invariante à direita $(n=\operatorname{dim} G)$. Daí:

$$
\begin{aligned}
\left\langle d \mu^{h} x, d \mu^{h} y\right\rangle_{h \cdot p} & =\int_{G_{x}}\left(d \mu^{g} d \mu^{h} x, d \mu^{g} d \mu^{h} y\right)_{g \cdot(h \cdot p)} w \\
& =\int_{G_{x}}\left(d \mu^{g h} x, d \mu^{g h} y\right)_{(g h) \cdot p)} w
\end{aligned}
$$

fazendo $f(g)=\left(d \mu^{g} x, d \mu^{g} y\right)_{g \cdot p}$, continuamos:

$$
\begin{aligned}
\int_{G_{x}} f(g h) w & =\int_{G_{x}} R_{h}^{*}(f w) \\
& =\langle x, y\rangle_{p}
\end{aligned}
$$

O resultado segue das propriedades da exponencial riemanniana (proposição 1.82) e do fato de isometria levar geodésica em geodésica.

Teorema 2.41. Seja $\mu: G \times M \longrightarrow M$ uma ação própria. Fixando $x \in M$, existe slice em $x$.

Demonstração: Vamos provar por afirmações, como segue:

Afirmação 2.42. $T_{x} G(x)$ é $d \mu^{h}$-invariante se $h \in G_{x}$.

Prova:

$$
\begin{aligned}
\mu(h, \exp (t \xi) \cdot x) & =\mu\left(h, \exp (t \xi) h^{-1} \cdot x\right) \\
& =\mu\left(h \exp (t \xi) h^{-1}, x\right)
\end{aligned}
$$

Derivando em $t=0$, temos

$$
d \mu^{h} \vec{\xi}(x)=\operatorname{Ad} \overrightarrow{(h)} \xi(x) \in T_{x} G(x)
$$

Da suposição de $G_{x}$ ser um grupo de isometrias juntamente com a afirmação 2.42 resulta que para

$$
v \in\left(T_{x} G(x)\right)^{\perp} \text { temos } d \mu^{h}(v) \in\left(T_{x} G(x)\right)^{\perp}
$$

ou seja,

$$
\left(T_{x} G(x)\right)^{\perp} \text { é também } d \mu^{h} \text { invariante para } h \in G_{x}
$$


Vamos definir

$$
S_{x}=\exp \left(\mathcal{B}_{\varepsilon}(0)\right)
$$

$\operatorname{com} \mathcal{B}_{\varepsilon}(0) \subset\left(T_{x} G(x)\right)^{\perp}$ e $\varepsilon>0$ suficientemente pequeno.

Do lema 2.40, item $i i i)$, resulta $\mu^{h}\left(S_{x}\right) \subset S_{x}$. Isto prova os itens a) e b) da definição de slice (definição 2.38).

Vamos provar o item c) da definição, supondo por absurdo que este não seja satisfeito.

Suponha por absurdo que existam sequências $\left(x_{n}\right)$ em $S_{x}$ e $\left(g_{n}\right)$ em $G$ tais que

$$
x_{n} \longrightarrow x
$$

e $\left(g_{n} \cdot x_{n}\right)$ é sequência em $S_{x}$ com

$$
g_{n} \cdot x_{n} \longrightarrow x, \text { mas } g_{n} \notin G_{x}
$$

Visto que a ação é própria, existe uma subsequência (a qual usamos o mesmo índice $n)\left(g_{n}\right)$ tal que

$$
g_{n} \longrightarrow g
$$

Da continuidade da ação, $g \in G_{x}$. Vamos definir

$$
\tilde{g}_{n}=g^{-1} g_{n}
$$

daí

$$
\tilde{g_{n}} \longrightarrow e, \tilde{g_{n}} \cdot x_{n} \in S_{x} \text { e } \tilde{g_{n}} \notin G_{x}
$$

Considere a variedade $C$ definida no lema 2.39.

Afirmação 2.43. Existe vizinhança $\mathcal{W}$ de $e$ em $G$ tal que, dado $g \in \mathcal{W}$, existe $h \in G_{x}$ e um único $c \in C, \operatorname{com} g=c \cdot h$.

Prova: Esta afirmação segue do fato de $\mathfrak{g}=\mathfrak{g}_{x} \oplus T_{e} C$ e do teorema da função inversa.

Por meio da afirmação 2.43 , temos que

$$
\tilde{g}_{n}=c_{n} \cdot h_{n}
$$

Do item b) da definição de slice (definição 2.38), resulta

$$
h_{n} \cdot x_{n} \in S_{x}
$$

Se $c_{n} \neq e$, sabemos pelo lema 2.39 que $c_{n} \cdot\left(h_{n} \cdot x_{n}\right) \notin S_{x}$, porém isto contraria o fato de $\tilde{g}_{n} \cdot x_{n} \in S_{x}$. 
Se $c_{n}=e$, temos que $\tilde{g}_{n}=c_{n} \cdot h_{n} \in G_{x}$, o que também contraria $\tilde{g}_{n} \notin G_{x}$.

\section{$2.4 \quad$ Fibrados Associados}

Seja $\rho: P \longrightarrow B$ um fibrado principal com grupo estrutural $H, F$ uma variedade de maneira que $H$ age sobre $F$.

Sabemos que $H$ age à direita de $P$ por uma ação livre e própria (proposição 2.23). Vamos definir uma ação:

$$
\begin{gathered}
H \times(P \times F) \longrightarrow(P \times F) \\
(h,(p, f)) \longmapsto\left(p \cdot h^{-1}, h \cdot f\right)
\end{gathered}
$$

Observação 2.44. A ação 2.12 dada acima é à esquerda livre e própria. Pelo teorema 2.31, o espaço de órbitas

$$
P \times_{H} F:=\left\{\left(p \cdot h^{-1}, h \cdot f\right): h \in H,(p, f) \in P \times F\right\}
$$

é uma variedade.

Definição 2.45. A variedade $P \times_{H} F$ definida na equação 2.13 acima é chamada produto torcido de $P$ e $F$.

Proposição 2.46. Seja

$$
\begin{aligned}
\pi: P \times_{H} F & \longrightarrow B \\
{[x, f] } & \longmapsto \rho(x)
\end{aligned}
$$

Então, $\left(P \times_{H} F, B, \pi, F, H\right)$ é fibrado.

Definição 2.47. O fibrado dado pela proposição 2.46 acima é chamado fibrado associado ao fibrado principal $\rho: P \longrightarrow B$ com fibra $F$.

Demonstração: Seja $S$ a variedade transversal a $(x) H$ e $\mathcal{U}:=(S) H$ a vizinhança $H$-invariante construida na demonstração do teorema 2.31 .

Defina

$$
\begin{gathered}
\psi: H \times(S \times F) \longrightarrow \mathcal{U} \times F \\
(h,(x, f)) \longmapsto\left(x \cdot h^{-1}, h \cdot f\right)
\end{gathered}
$$

observe que $\psi$ é difeomorfismo

Lema 2.48. Sejam $M$ e $N$ variedades. Suponha que $H$ age livre e propriamente à esquerda em $M$ e em $N$. Seja $\varphi: M \longrightarrow N$ um difeomorfismo $H$-invariante. Então, existe um único difeomorfismo $\tilde{\varphi}: M / H \longrightarrow N / H$ tal que $\tilde{\varphi} \circ \pi=\pi \circ \varphi$.

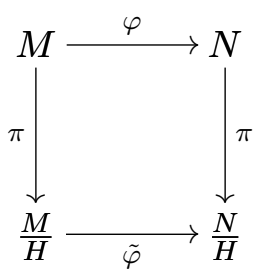


Segue do lema acima que existe um único difeomorfismo

$$
\begin{aligned}
\phi: S \times F & \longrightarrow \mathcal{U} \times_{H} F \\
(x, f) & \longmapsto[x, f]
\end{aligned}
$$

Por fim, para duas variedades transversais $S_{1}$ e $S_{2}$ defina:

$$
\begin{aligned}
\tilde{\phi}_{i}: \pi\left(S_{i}\right) \times F & \longrightarrow \mathcal{U} \times_{H} F \\
(b, f) & \longmapsto\left[\pi_{i}^{-1}(b), f\right]
\end{aligned}
$$

onde $\pi_{i}:=\left.\pi\right|_{S_{i}}$, com $i=1,2$. O resultado é consequência da afirmação 2.49 a seguir:

Afirmação 2.49.

$$
\tilde{\phi}_{2}^{-1} \circ \tilde{\phi}_{1}=(b, \theta(b) \cdot f)
$$

onde $\theta: \mathcal{V} \subset S_{2} \longrightarrow \mathcal{U} \subset S_{1}$ é definido como na demonstração do teorema 2.31.

Prova: De fato, se

$$
\left[x_{1}, f_{1}\right]=\left[x_{2}, f_{2}\right]
$$

concluimos que

$$
\left(x_{1} \cdot h^{-1}, h \cdot f_{1}\right)=\left(x_{2}, f_{2}\right)
$$

para algum $h$. Assim:

$$
\begin{gathered}
f_{2}=h \cdot f_{1} \\
x_{1}=x_{2} \cdot h \\
x_{1}=x_{2} \cdot \theta\left(x_{2}\right)
\end{gathered}
$$

Equações 2.16 e 2.17 e o fato da ação ser livre implica que

$$
\theta\left(x_{2}\right)=h
$$

Para obter o resultado, basta substituir a equação 2.18 na equação 2.15.

\subsection{Vizinhança Tubular}

Proposição 2.50. Seja $\mu: G \times M \longrightarrow M$ uma ação própria e $S_{x}$ slice em $x \in M$.

a) Então a ação

$$
\begin{gathered}
\alpha: G \times G \longrightarrow G \\
(a, b) \longmapsto a b
\end{gathered}
$$


induz uma única ação

$$
\tilde{\alpha}: G \times G / G_{x} \longrightarrow G / G_{x}
$$

tal que o diagrama

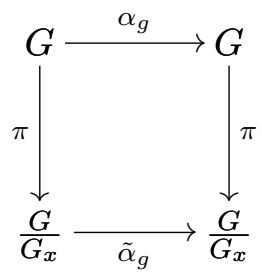

comuta.

b) Seja

$$
\beta: G \times\left(G \times S_{x}\right) \longrightarrow\left(G \times S_{x}\right)
$$

tal que

$$
\beta(a,(g, y))=(a g, y)
$$

Então, existe um único

$$
\tilde{\beta}: G \times\left(G \times_{G_{x}} S_{x}\right) \longrightarrow\left(G \times_{G_{x}} S_{x}\right)
$$

tal que o diagrama

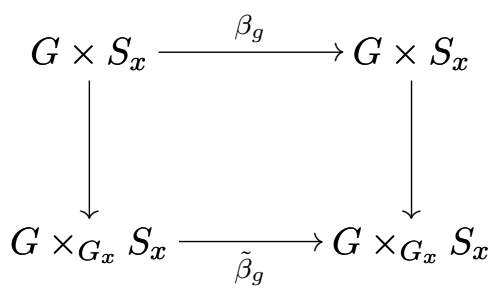

comuta.

c) O diagrama abaixo comuta: 


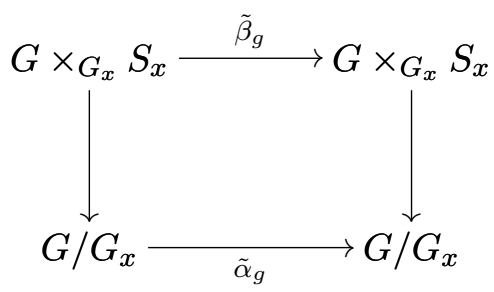

Teorema 2.51. Seja $\mu: G \times M \longrightarrow M$ uma ação própria e $S_{x_{0}}$ um slice. Então:

a) $G\left(S_{x_{0}}\right)$ é vizinhança $G$-invariante da órbita $G\left(x_{0}\right)$.

b)

$$
\begin{aligned}
\psi: G \times_{G_{x_{0}}} S_{x_{0}} & \longrightarrow G\left(S_{x_{0}}\right) \\
{[g, x] } & \longmapsto g \cdot x
\end{aligned}
$$

é um difeomorfismo $G$-equivariante, isto é, $g \cdot \psi[a, x]=\psi[g a, x]$.

Demonstração: Seja

$$
\begin{gathered}
\varphi: G \times S_{x_{0}} \longrightarrow M \\
(g, x) \longmapsto g \cdot x
\end{gathered}
$$

Afirmação 2.52. $d \varphi_{(g, x)}$ é sobrejetora

Prova: De fato,

$$
T_{x} M=d \mu_{x}(\mathfrak{g})+T_{x} S_{x_{0}}
$$

Efetuando

$$
\begin{aligned}
\varphi\left(g_{0} h, x\right) & =g_{0} \cdot(h \cdot x) \\
& =g_{0} \cdot \varphi(h, x) \\
& =\mu\left(g_{0}, \varphi(h, x)\right)
\end{aligned}
$$

resulta que

$$
d \varphi_{\left(g_{0}, x\right)}=d \mu^{g_{0}} d \varphi_{(e, x)}
$$

mas como $d \mu^{g_{0}}$ é inversível e $d \varphi_{(e, x)}$ é sobrejetor, podemos concluir a afirmação.

A afirmação 2.52 implica que $G\left(S_{x_{0}}\right)$ é aberto $G$-invariante.

Afirmação 2.53. $\varphi(g, x)=\varphi(h, y)$ se, e somente se, $h=g k^{-1}$ e $y=k \cdot x$, onde $k \in G_{x_{0}}$. Prova:

$(\Leftarrow)$ é imediatamente verdadeira.

Vamos verificar $(\Rightarrow)$. Se 


$$
g \cdot x=h \cdot y
$$

então

$$
y=k \cdot x
$$

onde

$$
k=h^{-1} g
$$

Usando que $y, x \in S_{x_{0}}$, onde $S_{x_{0}}$ é slice juntamente com a equação 2.19 resulta que

$$
k \in G_{x_{0}}
$$

Por fim, concluimos pela equação 2.20 que:

$$
h=g k^{-1}
$$

A afimação 2.53 e o fato de

$$
\pi: G \times S_{x_{0}} \longrightarrow G \times_{G_{x_{0}}} S_{x_{0}}
$$

ser fibrado principal implicam na existência de uma única aplicação

$$
\psi: G \times_{G_{x_{0}}} S_{x_{0}} \longrightarrow G\left(S_{x_{0}}\right)
$$

suave e bijetora tal que

$$
\varphi=\psi \circ \pi
$$

ou seja, que o diagrama

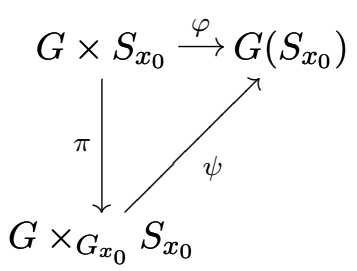

comuta.

Afirmação 2.54. $\psi$ é difeomorfismo.

Prova: Visto que $d \varphi$ é sobrejetora e $\varphi=\psi \circ \pi$, concluimos que $d \psi$ é sobrejetora. Por outro lado,

$$
\begin{aligned}
\operatorname{dim} G \times_{G_{x_{0}}} S_{x_{0}} & =\operatorname{dim} G / G_{x_{0}}+\operatorname{dim} S_{x_{0}} \\
& =\operatorname{dim} M \\
& =\operatorname{dim} S\left(G_{x_{0}}\right)
\end{aligned}
$$


Assim, $d \varphi$ é isomorfismo, e pelo teorema da função inversa $\psi$ é difeomorfismo local. Como $\psi$ é bijetora, é difeomorfismo global.

$\diamond$

Corolário 2.55. Seja $\psi$ definido no teorema 2.51. Então

$$
\psi\left[g_{0}, S_{x_{0}}\right]=S_{g_{0} \cdot x_{0}}
$$

Em outras palavras

$$
g_{0} \cdot S_{x_{0}}=S_{g_{0} \cdot x_{0}}
$$

Demonstração: Seja

$$
S:=\psi\left[g_{0}, S_{x_{0}}\right]
$$

Afirmação 2.56. $S$ atende ao item a) da definição de slice (definição 2.38).

Prova:

$$
\begin{aligned}
S & =\psi\left[g_{0}, S_{x_{0}}\right] \\
& =g_{0} \cdot \psi\left[e, S_{x_{0}}\right] \\
& =g_{0} \cdot S_{x_{0}}
\end{aligned}
$$

Logo, $S$ é também transversal às órbitas.

Para verificar o item b) da definição 2.38, devemos provar que

Afirmação 2.57.

$$
G_{g_{0} \cdot x}(S) \subset S
$$

Prova: Se $h \in G_{g_{0} \cdot x}$, segue da proposição 2.8 que

$$
h=g_{0} k g_{0}^{-1} \operatorname{com} k \in G_{x_{0}}
$$

Seja $y \in S$ :

$$
\begin{aligned}
h \cdot y & =h \cdot \psi\left[g_{0}, \tilde{y}\right] \\
& =\psi\left[h g_{0}, \tilde{y}\right] \\
& =\psi\left[g_{0} k g_{0}^{-1} g_{0}, \tilde{y}\right] \\
& =\psi\left[g_{0} k, \tilde{y}\right] \\
& =\psi\left[g_{0}, k^{-1} \cdot \tilde{y}\right] \in S
\end{aligned}
$$


Afirmação 2.58. Satisfaz o item c) da definição 2.38.

Prova: Suponha

$$
g \cdot \psi\left[g_{0}, x\right]=\psi\left[g_{0}, y\right]
$$

Então

$$
\left[g g_{0}, x\right]=\left[g_{0}, y\right]
$$

e temos

$$
\begin{gathered}
g_{0}=g g_{0} h^{-1} \\
y=h \cdot x
\end{gathered}
$$

$\operatorname{com} h \in G_{x}$. Assim

$$
h=g_{0}^{-1} g g_{0}
$$

implicando

$$
g=g_{0} h g_{0}^{-1} \in G_{g_{0} \cdot x}
$$

Observação 2.59. Existe uma única retração $G$-invariante

$$
r: G\left(S_{x_{0}}\right) \longrightarrow G\left(x_{0}\right)
$$

tal que

$$
r \circ \psi=\mu_{x_{0}} \circ \pi
$$

ou seja, de maneira que o diagrama abaixo comute.

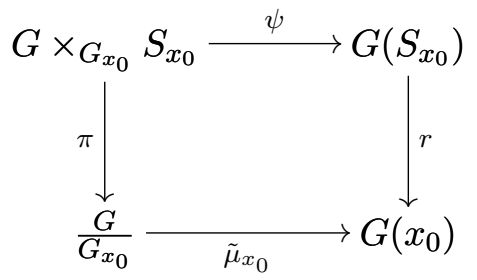

\subsection{Ações Isométricas}

As ações isométricas desempenham um papel essencial em nosso estudo, até porque o resultado final que apresentamos no cápítulo 3 supõe ações isométricas próprias. Mostraremos teoremas importantes com relação a ações isométricas próprias e estabeleceremos uma topologia em Iso( $M)$ (a saber, o conjunto das isometrias na variedade $M$ ) de maneira que este seja um grupo de Lie.

Definição 2.60 (Ação Isométrica). Uma ação riemanniana $\mu: G \times M \longrightarrow M$ é isométrica se $\mu^{g}$ é isometria para todo $g \in G$. 
Definição 2.61. Sejam $M, N$ espaços métricos e $\left(f_{n}\right)$ uma sequência de funções em $\mathcal{C}^{0}(M, N)$. Dizemos que $f_{n}$ converge na topologia da convergência compacta para $f \in \mathcal{C}^{0}(M, N)$ se $f_{n}$ converge uniformemente em cada compacto para $f$, ou seja

$$
\left.\left.f_{n}\right|_{K} \stackrel{u}{\longrightarrow} f\right|_{K}
$$

em cada compacto $K \subset M$.

Observação 2.62. .

1. Denotamos a convergência na topologia da convergência compacta por:

$$
f_{n} \stackrel{u_{c}}{\longrightarrow} f
$$

2. No caso de espaços métricos, tal topologia coincide com a topologia compacta aberta.

Teorema 2.63. Seja $M$ uma variedade riemanniana conexa. Então, o grupo de isometrias de $M$, denotado por Iso( $M)$, é grupo de Lie com a topologia da convergência compacta.

Proposição 2.64. Seja $\mu: G \times M \longrightarrow M$ uma ação à esquerda.

a) Se $g_{n} \longrightarrow g$, então $\mu^{g_{n}} \stackrel{u_{c}}{\longrightarrow} \mu^{g}$

b) Se a ação é própria e $\mu^{g_{n}} \stackrel{p t}{\longrightarrow} \mu^{g}$ (converge pontualmente), então $g_{n} \longrightarrow g$

\section{Demonstração:}

a) Por definição, $\mu^{g_{n}} \stackrel{u_{c}}{\longrightarrow} \mu^{g}$ se, dado um compacto $K$ e $\varepsilon>0$, existir $N_{K, \varepsilon}$ tal que

$$
d\left(g_{n}(x), g(x)\right)<\varepsilon
$$

para todo $n>N$ e $x \in K$.

Vamos supor que isto não seja verdade. Então, existe um compacto $K$ e $\varepsilon>0$ de maneira que para todo $n \in \mathbb{N}$, é possível encontrar $m>n$ e $x_{m} \in K$ com

$$
d\left(\mu^{g_{m}}\left(x_{m}\right), \mu^{g}\left(x_{m}\right)\right)>\varepsilon
$$

Como $K$ é compacto, podemos passar para uma subsequência convergente $\left(x_{m_{i}}\right)$ sendo $x_{m_{i}} \longrightarrow$ $x$. Tomando os índices $m_{i}$, temos $g_{m_{i}} \longrightarrow g$ e $x_{m_{i}} \longrightarrow x$, resultando

$$
g_{m_{i}} \cdot x_{m_{i}} \longrightarrow g \cdot x
$$

Por outro lado, 


$$
g \cdot x_{m_{i}} \longrightarrow g \cdot x
$$

Das equações 2.22 e 2.23 , temos que

$$
d\left(g_{m_{i}} \cdot x_{m_{i}}, g \cdot x_{m_{i}}\right) \longrightarrow 0
$$

contrariando a equação 2.21 .

b)

Afirmação 2.65. Suponha que $\mu^{k_{n}} \stackrel{p t}{\longrightarrow} \mu^{k}$. Então existe uma subsequência $\left(k_{n_{i}}\right)$ tal que $k_{n_{i}} \longrightarrow$ $k$.

Prova: Para cada $x$

$$
\mu^{k_{n}} \cdot x \longrightarrow \mu^{k} \cdot x
$$

Como a ação é própria, existe uma subsequência $k_{n_{i}} \longrightarrow t \in G$. Mas

$$
\begin{aligned}
& \mu^{k_{n_{i}}} \stackrel{p t}{\longrightarrow} \mu^{t} \\
& \mu^{k_{n_{i}}} \stackrel{p t}{\longrightarrow} \mu^{k}
\end{aligned}
$$

Das equações 2.24 e 2.25, resulta que $\mu^{k}(x)=\mu^{t}(x)$, para todo $x \in M$, e portanto $k=t$.

Agora, suponha por absurdo que $g_{n}$ não convirja para $g$. Então, existe uma subsequência $\left(g_{n_{i}}\right)$ tal que

$$
d\left(g_{n_{i}}, g\right)>\varepsilon
$$

Como $\mu^{g_{n_{i}}} \stackrel{p t}{\longrightarrow} \mu^{g}$, conseguimos por meio da afirmação 2.65 uma subsequência

$$
g_{n_{i j}} \longrightarrow g
$$

o que contraria a equação 2.26 .

Lema 2.66 (Partição da Unidade $G$-invariante). Seja $\mu: G \times M \longrightarrow M$ uma ação própria e $\left\{\mathcal{U}_{\alpha}\right\}$ uma cobertura de abertos localmente finita $G$-invariante. Então, existe uma partição da unidade $G$-invariante $\left\{f_{\alpha}\right\}$, estritamente subordinada a $\left\{\mathcal{U}_{\alpha}\right\}$. Em outras palavras, existe $\left\{f_{\alpha}\right\}$ sendo: 
a) $f_{\alpha}: M \longrightarrow \mathbb{R}, \quad f_{\alpha} \geq 0$

b) $\overline{\operatorname{supp}\left(f_{\alpha}\right)} \subset \mathcal{U}_{\alpha}$

c) $\sum_{\alpha} f_{\alpha}(x)=1$

d) $f_{\alpha}(g \cdot x)=f_{\alpha}(x)$

Teorema 2.67. Dada uma ação própria $\mu: G \times M \longrightarrow M$, existe uma métrica em $M$ de maneira que $\mu^{G}$ é subgrupo fechado de Iso $(M)$.

Demonstração: Vamos tomar abertos $G$-invariantes $\mathcal{U}_{\alpha}:=G\left(S_{x_{\alpha}}\right)$ e considerar $\left\{f_{\alpha}\right\}$ a partição da unidade $G$-invariante associada a $\left\{\mathcal{U}_{\alpha}\right\}$ (vide lema 2.66).

Afirmação 2.68. A estrutura definida em $\left.T M\right|_{S_{x_{\alpha}}}$ por

$$
\langle x, y\rangle_{p}=\int_{G_{x_{\alpha}}}\left(d \mu^{g} x, d \mu^{g} y\right)_{g \cdot p} w
$$

onde $w$ é uma forma invariante à direita e ( , ) é métrica qualquer, é $G_{x_{\alpha}}$-invariante.

Prova: Usamos aqui o mesmo raciocínio aplicado na demonstração do lema 2.40 .

Vamos definir uma métrica em $\mathcal{U}_{\alpha}$ por:

$$
\left\langle d \mu^{g} x, d \mu^{g} y\right\rangle_{g \cdot s}:=\langle x, y\rangle
$$

onde $s \in S_{x_{\alpha}}$

Como $\langle$,$\rangle é G_{x_{\alpha}}$-invariante e $G_{s} \subset G_{x_{\alpha}}$ para $s \in S_{x_{\alpha}}$, o produto definido na equação 2.27 acima está bem definido.

Por fim, basta definir

$$
\langle,\rangle:=\sum_{\alpha} f_{\alpha}\langle,\rangle
$$

$\mu^{G}$ se torna então um grupo de isometrias. Falta provar que é fechado em $\operatorname{Iso}(M)$.

Suponha que

$$
\mu^{g_{n}} \stackrel{u_{c}}{\longrightarrow} f \in \operatorname{Iso}(M)
$$

Então

$$
g_{n} \cdot x \longrightarrow f \cdot x
$$

como a ação é própria, existe subsequência $\left(g_{n_{i}}\right)$ tal que

$$
g_{n_{i}} \longrightarrow g
$$


Do item a) da proposição 2.64

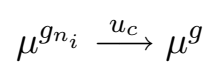

Das equações 2.28 e 2.29 concluimos que

$$
g=f
$$

Teorema 2.69 (Ascoli-Arzelá). Sejam $X, Y$ espaços métricos compactos e $\mathcal{C}^{0}(X, Y)$ dotado da métrica

$$
d(f, g):=\sup _{x \in X} d(f(x), g(x))
$$

Seja $\mathcal{F}$ uma família em $\mathcal{C}^{0}(X, Y)$. Então, $\mathcal{F}$ é relativamente compacta se a família $\mathcal{F}$ é equicontínua, isto é, se dado $\varepsilon>0$, existe $\delta>0$ tal que, tomando $x, \tilde{x} \in X \operatorname{com} d(x, \tilde{x})<\delta$, então $d(f(x), f(\tilde{x}))<$ $\varepsilon$ para todo $f \in \mathcal{F}$.

Para uma demonstração do teorema de Ascoli-Arzelá, consultar [L1], pg. 244.

Teorema 2.70. Seja $G \subset$ Iso $(M)$ um subgrupo fechado e $M$ uma variedade riemanniana conexa.A $a c ̧ \tilde{a} o$

$$
\begin{gathered}
\mu: G \times M \longrightarrow M \\
(g, x) \longmapsto g \cdot x
\end{gathered}
$$

é própria.

Demonstração: Vamos primeiro considerar o caso em que $M$ é completa.

Sejam

$$
\begin{gathered}
g_{n} \cdot x_{n} \longrightarrow y \\
x_{n} \longrightarrow x
\end{gathered}
$$

e $n_{0}$ tal que, para $n>n_{0}$

$$
\begin{gathered}
d\left(y, g_{n} \cdot x_{n}\right)<\frac{\varepsilon}{2} \\
d\left(x, x_{n}\right)<\frac{\varepsilon}{2}
\end{gathered}
$$

Fixe $r_{1}>0$ e tome a bola fechada $K:=\overline{\mathcal{B}}_{r_{1}}(x)$. 
Afirmação 2.71.

$$
d\left(g_{n} \cdot K, y\right) \leq \varepsilon+r_{1}
$$

Prova: De fato, tomando $z \in K$ :

$$
\begin{aligned}
d\left(g_{n} \cdot z, y\right) & \leq d\left(y, g_{n} \cdot x_{n}\right)+d\left(g_{n} \cdot x_{n}, g_{n} \cdot z\right) \\
& \leq \bar{\varepsilon}+d\left(x_{n}, z\right) \\
& \leq \frac{\varepsilon}{2}+d\left(x_{n}, x\right)+d(x, z) \\
& \leq \frac{\varepsilon}{2}+\frac{\varepsilon}{2}+r_{1} \\
& \leq \varepsilon+r_{1}
\end{aligned}
$$

O fato de $g_{n}$ ser isometria implica que

Afirmação 2.72. $\left(g_{n}\right)$ é família equicontínua

Como a variedade é completa, as bolas fechadas são compactas. Segue das afirmações 2.71 e 2.72, juntamente com o teorema de Ascoli-Arzelá (teorema 2.69) que existe uma subsequência $\left(g_{n_{i}}\right)$ convergindo uniformemente em $K$ para uma função contínua $g \in \mathcal{C}^{0}(K, M)$. Visto que $g_{n_{i}} \in I s o(M)$, podemos concluir que $g \in I s o(M)$. Além disso, $g \in G$, pois $G$ é fechado.

Por um argumento de diagonal de Cantor, podemos obter uma subsequência $\left(g_{n_{i j}}\right)$ convergente em cada compacto $\overline{\mathcal{B}}_{r_{j}}(x)$.

Caso geral: Suponha

$$
\begin{gathered}
x_{n} \longrightarrow x \\
g_{n} \cdot x_{n} \longrightarrow y
\end{gathered}
$$

então, para todo $\varepsilon>0$ é possível encontrar $n_{0}$ tal que, sendo $n>n_{0}$,

$$
\begin{gathered}
d\left(x_{n}, x\right)<\varepsilon \\
d\left(g_{n} \cdot x_{n}, y\right)<\varepsilon
\end{gathered}
$$


Como $g_{n}$ é isometria, temos

$$
\begin{aligned}
d\left(g_{n} \cdot x, y\right) & \leq d\left(g_{n} \cdot x, g_{n} \cdot x_{n}\right)+d\left(g_{n} \cdot x_{n}, y\right) \\
& \leq d\left(x, x_{n}\right)+d\left(g_{n} \cdot x_{n}, y\right) \\
& <\frac{\varepsilon}{2}+\frac{\varepsilon}{2} \\
& =\varepsilon
\end{aligned}
$$

e portanto

$$
g_{n} \cdot x \longrightarrow y
$$

O resultado segue do lema abaixo:

Lema 2.73. Seja $\left(g_{n}\right)$ uma sequência em Iso $(M)$ e suponha que exista $p \in M$ tal que $\left(g_{n}(p)\right)$ converge. Então, $\left(g_{n}\right)$ admite uma subsequência $\left(g_{n_{i}}\right)$ tal que

$$
g_{n_{i}} \stackrel{u_{c}}{\longrightarrow} g \in \operatorname{Iso}(M)
$$

Para uma demonstração deste lema, consultar [KN], pg. 46.

Observação 2.74. Existe um grupo de isometrias fechado de uma variedade $M$ de dimensão finita que não age propriamente.

Seja $M=\ell^{2}$, com o produto

$$
\langle x, y\rangle=\sum_{i=1}^{\infty} x_{i} y_{i}
$$

e $G=O_{n}$, grupo ortogonal.

Se $G$ agisse propriamente então $O_{n}=G_{\overrightarrow{0}}$ seria grupo compacto, uma contradição pois tomando

$$
T_{k}\left(a_{1}, a_{2}, \ldots\right)=\left(a_{2}, a_{3}, \ldots, a_{k}, a_{1}, a_{k+1}, \ldots\right)
$$

podemos notar que $T_{k}\left(e_{1}\right)$, com $e_{1}=(1,0,0, \ldots)$ não admite subsequência convergente, pois

$$
\left\|T_{n}\left(e_{1}\right)-T_{m}\left(e_{1}\right)\right\|=2
$$

para quaisquer $n \neq m$.

\section{7 Órbita Principal}

Agora vamos definir o conceito de órbita principal e de representação isotrópica e slice. A partir da definição de campo equivariante normal, vamos mostrar que o fibrado normal de órbitas principais é trivial.

Definição 2.75 (Órbita Principal). Dada uma ação própria $\mu: G \times M \longrightarrow M$, uma órbita $G(x)$ é principal se existir uma vizinhança $\mathcal{U}_{x}$ de $x$ em $M$ onde, para todo $y \in \mathcal{U}_{x}$, existe $g \in G$ tal que $G_{x} \subset g G_{y} g^{-1}$. 
Observação 2.76. Seja $G(x)$ órbita principal e $y \in S_{x}$. Então, $G_{y}=G_{x}$.

De fato, $G_{y} \subset G_{x}$, pela definição de slice, e como $G(x)$ é órbita principal

$$
G_{y} \subset G_{x} \subset g G_{y} g^{-1}
$$

Como $G_{y}$ é compacto,

$$
G_{y}=g G_{y} g^{-1}
$$

e portanto $G_{y}=G_{x}$.

Definição 2.77. Seja $\mu: G \times M \longrightarrow M$ uma ação riemanniana própria. Definimos representação isotrópica em $x \in M$ como a aplicação

$$
\begin{aligned}
G_{x} & \longrightarrow G L\left(T_{x} M\right) \\
g & \longmapsto d \mu^{g}
\end{aligned}
$$

Observação 2.78. Como a representação isotrópica deixa $T_{x} G(x)$ invariante, também deixa $T_{x} G(x)^{\perp}$ invariante.

Definição 2.79. Seja $\mu: G \times M \longrightarrow M$ uma ação riemanniana própria. Definimos representação slice por:

$$
\begin{aligned}
G_{x} & \longrightarrow \mathcal{O}\left(T_{x} G(x)\right)^{\perp} \\
g & \longmapsto d \mu^{g}
\end{aligned}
$$

Observação 2.80. Para uma ação isométrica própria,

$$
S_{x}=\exp _{x}\left(\mathcal{B}_{\varepsilon}(0)\right)
$$

onde $\mathcal{B}_{\varepsilon}(0) \subset T_{x} G(x)^{\perp}$.

Proposição 2.81. Seja $\mu: G \times M \longrightarrow M$ uma ação riemanniana própria. $G(x)$ é órbita principal se, e somente se, a representação slice é trivial (isto é, $d \mu^{g}=I$ para todo $g \in G_{x}$ ).

Demonstração: Se $G(x)$ é órbita principal, pela observação $2.76, G_{x}=G_{y}$ para todo $y \in S_{x}$. Logo, a representação slice é trivial.

Supondo que a representação slice seja trivial, temos que

$$
d \mu^{g} v=v
$$

para todo $g \in G_{x}$ e para todo $v \in T_{x} S_{x}$

Assim

$$
\begin{aligned}
\mu^{g} \exp _{x}(v) & =\exp _{x}\left(d \mu^{g} v\right) \\
& =\exp _{x}(v)
\end{aligned}
$$


Isto implica que $G_{x} \subset G_{y}$ para todo $y \in S_{x}$. Visto que $G_{y} \subset G_{x}$, temos $G_{y}=G_{x}$ para todo $y \in S_{x}$. Logo $G(x)$ é órbita principal.

Proposição 2.82. Seja $\mu: G \times M \longrightarrow M$ uma ação riemanniana própria, $G(x)$ órbita principal $e v \in T_{x} G(x)^{\perp}$. Então

$$
\vec{V}_{g \cdot x}:=d\left(\mu^{g}\right)_{x} v_{x}
$$

é um campo bem definido.

Demonstração: Se

$$
g \cdot x=k \cdot x
$$

então

$$
\left(k^{-1} g\right) \cdot x=x
$$

$\log 0$

$$
k^{-1} g \in G_{x}
$$

e por consequência existe $h \in G_{x}$ com

$$
g=k h
$$

Como $G(x)$ é principal, segue da proposição 2.81 que $\left.d \mu^{h}\right|_{T_{x} S_{x}}=I$, e assim

$$
\begin{aligned}
\left.d \mu^{g}\right|_{T_{x} S_{x}} & =\left.d \mu^{k h}\right|_{T_{x} S_{x}} \\
& =\left.d \mu^{k} d \mu^{h}\right|_{T_{x} S_{x}} \\
& =\left.d \mu^{k}\right|_{T_{x} S_{x}}
\end{aligned}
$$

Definição 2.83. Um campo $\vec{V}$ ao longo de $G(x)$ é chamado equivariante normal se:

a) $d \mu^{g} \vec{V}_{y}=\vec{V}_{g \cdot y}$, para quaisquer $g \in G$ e $y \in G(x)$

b) $\vec{V}_{y} \in T_{y} G(x)^{\perp}$

Corolário 2.84. Seja $\mu: G \times M \longrightarrow M$ uma ação riemanniana própria e $G(x)$ uma órbita principal. Então $G(x)$ admite um referencial normal global do fibrado normal. Em particular, o fibrado normal de $G(x)$ é trivial.

Proposição 2.85. Seja $\mu: G \times M \longrightarrow M$ uma ação riemanniana própria, $G(x)$ uma órbita principal e $\vec{V}$ um campo normal equivariante. Então: 
a) $A_{\vec{V}_{g \cdot x}}=d \mu^{g} A_{\vec{V}_{x}} d \mu^{g^{-1}}$, onde $A_{v}(w)=-\nabla_{w}^{t} v$

b) As curvaturas de $G(x)$ ao longo de $\vec{V}$ são constantes.

c) $\left\{\exp _{y}\left(\vec{V}_{y}\right): y \in G(x)\right\}$ é uma órbita.

\section{Demonstração:}

a)

$$
\begin{aligned}
\left\langle d \mu^{g^{-1}} A_{\vec{V}_{g} \cdot x} d \mu^{g} w, z\right\rangle_{x} & =\left\langle A_{\vec{V}_{g} \cdot x} d \mu^{g} w, d \mu^{g} z\right\rangle_{g \cdot x} \\
& =\left\langle-\nabla_{d \mu^{g} w} d \mu^{g} \vec{V}_{x}, d \mu^{g} z\right\rangle_{g \cdot x} \\
& =\left\langle-d \mu^{g} \nabla_{w} \vec{V}_{x}, d \mu^{g} z\right\rangle_{g \cdot x} \\
& =-\left\langle\nabla_{w} \vec{V}_{x}, z\right\rangle_{x} \\
& =\left\langle A_{\vec{V}_{x}} w, z\right\rangle_{x}
\end{aligned}
$$

b) Temos que $A_{\vec{V}_{x}} k=\lambda k$. Disso resulta

$$
d \mu^{g^{-1}} A_{\vec{V}_{g \cdot x}} k=\lambda k
$$

e, por fim

$$
A_{\vec{V}_{g \cdot x}}\left(d \mu^{g} k\right)=\lambda\left(d \mu^{g} k\right)
$$

c)

$$
\begin{aligned}
\exp _{g \cdot x}\left(\vec{V}_{g \cdot x}\right) & =\exp _{g \cdot x}\left(d \mu^{g} \vec{V}_{x}\right) \\
& =g \cdot \exp _{x}(\vec{V})
\end{aligned}
$$

\subsection{Campos de Killing}

Definição 2.86 (Campos de Killing). Seja $M$ uma variedade riemanniana, $\vec{X} \in \Xi(M)$ e

$$
\varphi^{X}:(-\varepsilon, \varepsilon) \times \mathcal{U} \longrightarrow M
$$

o fluxo de $\vec{X}$.

$\vec{X}$ é chamado Campo de Killing se

$$
\varphi_{t_{0}}^{X}: \mathcal{U} \longrightarrow M
$$

é isometria para todo $t_{0} \in(-\varepsilon, \varepsilon)$ 
Proposição 2.87. Seja $\mu: G \times M \longrightarrow M$ uma ação isométrica própria. Então, uma geodésica que é ortogonal a uma órbita é também ortogonal a todas as órbitas que encontra.

Demonstração: Seja $\alpha$ uma geodésica tal que

$$
\alpha^{\prime}(0) \in T_{\alpha(0)} G(\alpha(0))^{\perp}
$$

Tome $\xi \in \mathfrak{g}$ e seja

$$
\vec{\xi}_{p}=\left.\frac{d}{d t}(\exp (t \xi) \cdot p)\right|_{t=0}
$$

conforme definido na proposição 2.10 .

Por hipótese,

$$
\left\langle\vec{\xi}_{\alpha(0)}, \alpha^{\prime}(0)\right\rangle=0
$$

Desejamos mostrar que, para todo $t$,

$$
\left\langle\vec{\xi}_{\alpha(t)}, \alpha^{\prime}(t)\right\rangle=0
$$

Como $\vec{\xi}$ é campo de Killing,

$$
\left\langle\nabla_{\alpha^{\prime}} \vec{\xi}, \alpha^{\prime}\right\rangle+\left\langle\nabla_{\alpha^{\prime}} \vec{\xi}, \alpha^{\prime}\right\rangle=0
$$

e por consequência

$$
\left\langle\nabla_{\alpha^{\prime}} \vec{\xi}, \alpha^{\prime}\right\rangle=0
$$

Assim, usando a equação 2.30:

$$
\begin{aligned}
\frac{d}{d t}\left\langle\vec{\xi}_{\alpha(t)}, \alpha^{\prime}(t)\right\rangle & =\left\langle\nabla_{\alpha^{\prime}} \vec{\xi}, \alpha^{\prime}\right\rangle+\left\langle\vec{\xi}, \nabla_{\alpha^{\prime}} \alpha^{\prime}\right\rangle \\
& =0
\end{aligned}
$$

Como supomos

$$
\left\langle\vec{\xi}_{\alpha(0)}, \alpha^{\prime}(0)\right\rangle=0
$$

resulta que, para todo $t$

$$
\left\langle\vec{\xi}_{\alpha(t)}, \alpha^{\prime}(t)\right\rangle=0
$$

Da arbitrariedade na escolha de $\xi$ resulta a veracidade da proposição. 
Teorema 2.88 (Teorema da Órbita Principal). Seja $\mu: G \times M \longrightarrow M$ uma ação própria onde $M$ é conexa. Então:

a) O conjunto dos pontos contidos em órbitas principais (denotado por $M_{p}$ ) é não-vazio, aberto e denso em $M$.

b) O conjunto $M_{p} / G$ é variedade conexa.

c) Sejam $G(x), G(y)$ duas órbitas principais. Então $G_{x}=g G_{y} g^{-1}$, para algum $g \in G$.

Demonstração: Ao longo da demonstração, vamos considerar $M$ dotado de uma métrica em que $\mu^{G}$ se torna subgrupo fechado de $I s o(M)$ (isto nos é garantido pelo teorema 2.67).

O item a) será provado no lema abaixo:

Lema 2.89. $M_{p}$ é conjunto não-vazio, aberto e denso em $M$.

Prova: Vamos mostrar por meio de afirmações:

Afirmação 2.90. $M_{p} \neq \emptyset$

Prova: Visto que os grupos de isotropia são compactos, podemos escolher $x \in M$ tal que $G_{x}$ tem a menor dimensão possível e para tal dimensão o menor número de componentes conexas.

Seja $S_{x}$ o slice em $x$. Pela definição de slice, $G_{y} \subset G_{x}$, para $y \in S_{x}$ e, pela escolha de $x$, temos $G_{y}=G_{x}$. Como $G\left(S_{x}\right)$ é aberto, $G(x)$ é órbita principal.

Afirmação 2.91. $M_{p}$ é aberto

Prova: Seja $x \in M_{p}$. Então, para todo $y \in S_{x}$, temos $G_{y}=G_{x}$. Assim $G\left(S_{x}\right) \subset M_{p}$.

Afirmação 2.92. $M_{p}$ é denso

Prova: Seja $x \in M, \mathcal{U}$ vizinhança de $x$ em $M$ e $S_{x}$ slice em $x$.

Escolha $y \in G\left(S_{x}\right) \cap \mathcal{U}$ tal que $G_{y}$ tem a menor dimensão e para tal dimensão o menor número de componentes conexas.

Seja $z \in G\left(S_{y}\right) \cap \mathcal{U} \cap G\left(S_{x}\right)$. Então, $G_{g^{-1} \cdot z} \cap G_{y}$. A escolha de $y$ implica que $G_{g^{-1} \cdot z}=G_{y}$ e portanto $G(y)$ é principal. 
Lema 2.93. Se $\left(M-M_{p}\right) / G$ não desconecta localmente por caminhos $M_{p} / G$ e $M / G$ é conexo, então $M_{p} / G$ é conexo por caminhos.

Prova: Sejam $y, z \in M_{p} / G$ e $\alpha$ uma curva em $M / G$ com $\alpha(0)=y$ e $\alpha(1)=z$ (isto é possível porque $M / G$ é conexo por caminhos).

Seja $\mathcal{V}_{1}, \ldots, \mathcal{V}_{n}$ uma cobertura aberta de $M / G$ que não desconecta caminhos. Sabemos que $M_{p} / G$ é aberto e denso em $M / G$, assim existe

$$
p_{i} \in \mathcal{V}_{i} \cap \mathcal{V}_{i+1} \cap M_{p} / G
$$

Usando a propriedade que $\mathcal{V}_{i}$ não desconecta por caminhos $M_{p} / G$, podemos encontrar uma curva $\beta$ em $M_{p} / G$ tal que $y, z, p_{i} \in \beta$.

Lema 2.94. $\left(M-M_{p}\right) / G$ não desconecta localmente por caminhos $M_{p} / G$.

Prova: O fato de $S_{x} / G_{x} \cong G\left(S_{x}\right) / G$ implica que, para provar o lema 2.94 , basta mostrar que :

Afirmação 2.95. $\left(S_{x}-\left(S_{x}\right)_{p}\right) / G_{x}$ não desconecta $\left(S_{x}\right)_{p} / G_{x}$.

Usando a definição de representação isotrópica e a afirmação 2.95 , concluimos que para provar o lema 3, basta provar:

Afirmação 2.96.

Seja $K$ um grupo compacto de isometrias agindo em $\mathbb{R}^{n}$. Então $\left(\mathbb{R}^{m}-\mathbb{R}_{p}^{n}\right) / K$ não desconecta $\mathbb{R}_{p}^{n} / K$

Prova: Provemos por indução na dimensão $m$.

Se $m=1$, temos $K=\{-1,1\}$ e $\mathbb{R} / K=[0, \infty)$.

Vamos supor que a afirmação 2.96 seja verdadeira para $m<n$.

Considerando a ação de $K$ em $S^{n-1}$ (esfera unitária), a hipótese de indução, a definição de representação slice, e a afirmação 2.90 temos que

$$
\left(S^{n-1}-S_{p}^{n-1}\right) / K
$$

não desconecta localmente $S^{n-1} / K$.

Seja $x, y \in \mathbb{R}_{p}^{n}$ e $\tilde{x}$ o ponto contido na reta ligando 0 a $x$ tal que $\tilde{x}$ e $y$ estão na mesma esfera.

O fato de $\left(S^{n-1}-S_{p}^{n-1}\right) / K$ não desconectar localmente $S^{n-1} / K$ e o lema 2.89 implicam que $G(\tilde{x})$ e $G(y)$ podem ser conectados por uma curva $\alpha$ em $\mathbb{R}_{p}^{n} / K$. 
Por outro lado, os pontos ao longo da reta que ligam $x$ a $\tilde{x}$ têm a mesma isotropia (representação linear), assim $G(x)$ e $G(\tilde{x})$ estão conectados em $\mathbb{R}_{p}^{n} / K$ por uma curva $\beta$. Concluimos que $\alpha * \beta$ conecta $G(x)$ a $G(y)$ em $\mathbb{R}_{p}^{n} / K$.

O item b) será provado no lema abaixo:

Lema 2.97. $M_{p} / G$ é variedade conexa.

Prova: Os lemas 2.93 e 2.94 implicam que $M_{p} / G$ é conexo por caminhos. O fato de $M_{p} / G$ ser conexo por caminhos e a representação isotrópica em $S_{x}$ ser trivial (proposição 2.81) junto com o mesmo argumento usado na demonstração do teorema 2.31 implica que $M_{p} / G$ é variedade conexa.

O item c) é consequência do item b).

\subsection{Tipos de Órbita}

Definição 2.98. Seja $\mu: G \times M \longrightarrow M$ uma ação própria.

a) Duas órbitas $G(x)$ e $G(y)$ são do mesmo tipo se existir $g \in G$ tal que

$$
G_{x}=g G_{y} g^{-1}=G_{g \cdot y}
$$

b) Se $H$ é grupo de isometrias de algum ponto de $M$ dizemos que $G(x)$ é do tipo $(H)$ se existir $g \in G$ tal que

$$
g G_{x} g^{-1}=H
$$

c) $\left(G_{x}\right) \leq\left(G_{y}\right)$ se existir $g \in G$ tal que

$$
g G_{y} g^{-1} \subset G_{x}
$$

d) Uma órbita é chamada regular se $\operatorname{dim} G(x)$ é igual à dimensão da órbita principal.

e) Uma órbita que não é regular é chamada singular.

f) Uma órbita regular que não é principal é chamada excepcional. 
Teorema 2.99. Seja $\mu: G \times M \longrightarrow M$ uma ação própria. Então, dado um ponto $p \in M$, existe uma vizinhança $G$-invariante $\mathcal{U}$ de $p$ de maneira que $\mathcal{U}$ tem somente um número finito de tipos de órbitas. Em particular, se $M$ é compacta, $M$ em um número finito de tipos de órbitas.

Demonstração: Considere $S_{p}$ o slice em $p$ e defina $\mathcal{U}:=G\left(S_{p}\right)$. Considere também $M$ dotado de métrica tal que $\mu^{G} \subset I s o(M)$.

Afirmação 2.100. Se duas $G_{p}$-órbitas $G_{p}(x)$ e $G_{p}(y)$, com $x, y \in S_{p}$, são do mesmo tipo então as duas $G$-órbitas $G(x)$ e $G(y)$ são do mesmo tipo.

Observação 2.101. A afirmação 2.100 implica que o número de tipos de $G$-órbitas em $\mathcal{U}$ é menor ou igual ao número de tipos de $G_{p}$-órbitas em $S_{p}$.

Observação 2.102. $G_{p}$ age propriamente em $S_{p}$, pois $G_{p}$ é compacto.

Faremos uma demonstração por indução na dimensão de $M$.

Quando $\operatorname{dim} M=0$, o resultado é trivialmente verdadeiro.

Hipótese de Indução: O teorema é verdadeiro para $M \operatorname{com} \operatorname{dim} M=k<n$.

Desejamos mostrar que o teorema é verdadeiro para $k=n$.

Se $\operatorname{dim} S_{p}<n$, podemos utilizar a observação 2.101 e a hipótese de indução para encerrar a demonstração.

Vamos supor que $\operatorname{dim} S_{p}=n$.

Neste caso, $G_{p}=G$ e $d \mu^{G_{p}}$ age em $T_{p} M \cong \mathbb{R}^{n}$ como subgrupo de $\mathcal{O}_{n}(\mathbb{R})$.

Note que $\mathcal{O}_{n}(\mathbb{R})$ deixa $S^{n-1}$ invariante e, por hipótese de indução, $S^{n-1}$ tem número finito de tipos de órbitas. Os grupos de isotropia (e assim os tipos isotrópicos) são constantes em cada linha que contém 0 , e portanto a ação da representação isotrópica tem no máximo uma órbita a mais em $S_{p}$ do que em $S^{n-1}$, e consequentemente um número finito.

Proposição 2.103. Seja $\mu: G \times M \longrightarrow M$ uma ação riemanniana própria e $H$ um subrupo compacto de G. Então, cada componente de

$$
M^{H}=\{x \in M: h \cdot x=x, \forall h \in H\}
$$

é variedade mergulhada totalmente geodésica isto é, toda geodésica de $M^{H}$ é geodésica de $M$.

Observação 2.104. Componentes conexas de $M^{H}$ podem ter dimensão diferentes. 
Definição 2.105. Seja $\mu: G \times M \longrightarrow M$ uma ação própria. $G(x)$ e $G(y)$ são do mesmo tipo local se existir um difeomorfismo equivariante

$$
\psi: S\left(S_{x}\right) \longrightarrow S\left(S_{y}\right)
$$

Observação 2.106. $G(x)$ e $G(y)$ são do mesmo tipo local se:

1. são do mesmo tipo.

2. existir um difeomorfismo

$$
\varphi: S_{x} \longrightarrow S_{y}
$$

com

$$
\varphi \cdot g=g \cdot \varphi
$$

para $g \in G_{x}$.

Observação 2.107. Relação de tipo local é mais fina do que a de tipo.

Definição 2.108. Seja $H \in G$ um subgrupo. Definimos o normalizador de $H$ como

$$
N(H):=\left\{g \in G: g H g^{-1}=H\right\}
$$

Teorema 2.109. Seja $\mu: G \times M \longrightarrow M$ uma ação própria e $x \in M$. Denote por $M_{\tilde{x}}$ o conjunto dos pontos com o mesmo tipo de $x$ e $M_{\hat{x}}$ o conjunto dos pontos com mesmo tipo local de $x$. Então:

a) $M_{\hat{x}}$ é aberto e fechado em $M_{\tilde{x}}$.

b) $M_{\hat{x}} \cap M^{G_{x}}$ é aberto em $M^{G_{x}}$. Além disso, é variedade $N\left(G_{x}\right)$-invariante.

c) $A$ ação de $N\left(G_{x}\right) / G_{x}$ em $M_{\hat{x}} \cap M^{G_{x}}$ é livre e própria.

d) $M_{\hat{x}}$ é variedade $G$-equivariante difeomorfa

$$
G / G_{x} \times_{N\left(G_{x}\right) / G_{x}}\left(M_{\hat{x}} \cap M^{G_{x}}\right)
$$

Definição 2.110. Uma estratificação de uma variedade $M$ é uma partição localmente finita por subvariedades $\left\{M_{i}\right\}_{i \in I}$ de $M$, chamadas estratos tal que:

a) Para cada $i \in I$,

$$
\bar{M}_{i}=M_{i} \cup\left(\cup_{j \in I_{i}} M_{j}\right)
$$

onde $I_{i} \subset I-\{i\}$. 
b) $\operatorname{dim} M_{j}<\operatorname{dim} M_{i}$ para cada $j \in I_{i}$.

Teorema 2.111. Seja $\mu: G \times M \longrightarrow M$ uma ação própria. As componentes conexas dos tipos de órbitas de $M$ formam uma estratificação de $M$. 


\section{Capítulo 3}

\section{A Conjectura de Terng-Palais}

Este é o capítulo final desta dissertação, onde usando o instrumental teórico apresentado nos capítulos 1 e 2 vamos compreender e demonstrar o Teorema 3.8, o qual chamamos de Conjectura de Palais-Terng. Segundo [HOL] este teorema garante que se a distribuiçao normal de uma ação propria é integravel, então a ação é polar, i.e., existem subvariedades imersas, completas, totalmente geodésicas que encontram todas as orbitas ortogonalmente

Algumas provas distintas foram feitas para esta conjectura, sendo uma delas apresentada no próprio artigo [HOL], mas para demonstrá-la optamos por adaptar a prova de Alexandrino ( [A]); em seu artigo, apresenta uma versão mais geral válida para folheações riemannianas singulares. Como lidamos com um caso particular, optamos por adaptar esse resultado ao caso de órbitas regulares de ações isométricas próprias.

$\mathrm{Na}$ organização desse capítulo iniciamos apresentando alguns fatos de imersões riemannianas que serão utilizados, mas sem a preocupação de demonstrá-los, visto que facilmente podem ser encontrados em qualquer livro-texto de Geometria Riemanniana. A seguir partimos para a demonstração da conjectura, à qual dedicamos uma seção.

\subsection{Fatos sobre Imersões Riemannianas}

Elencamos aqui alguns fatos sobre imersões riemannianas necessárias para o entendimento da demonstração do teorema 3.8. Para uma demonstração cuidadosa desses fatos, consultar o capítulo 6 de $[\mathrm{dC} 1]$.

Observação 3.1. Dada uma imersão $f: N \longrightarrow M$, onde $M$ possui uma estrutura riemanniana, podemos induzir em $N$ uma métrica por:

$$
\langle u, v\rangle_{p}:=\left\langle d f_{p} u, d f_{p} v\right\rangle_{f(p)}
$$

Neste caso, $\langle,\rangle_{p}$ é chamada métrica induzida por $f$ em $N$, e $f$ é chamada imersão isométrica.

Observação 3.2. Dada uma imersão $f: N \longrightarrow M$, como em cada $p \in N$ temos um mergulho $f: \mathcal{U} \longrightarrow f(\mathcal{U})$, onde $\mathcal{U}$ é uma vizinhança de $p$ em $N$, temos a seguinte decomposição:

$$
T_{p} M=T_{p} N \oplus\left(T_{p} N\right)^{\perp}
$$

Costumamos também denotar $\left(T_{p} N\right)^{\perp}$ por $\nu_{p} N$ 
Definição 3.3. Definimos $\nu_{p} N$ da observação 3.2 acima como o espaço normal a $N$ em $p$. Mais geralmente, sendo $\nu N:=\cup_{p \in N} \nu_{p} N$, temos que $(\nu N, \pi, N)$ é o fibrado normal a $N$.

Observação 3.4. Supondo $f: N \longrightarrow M$ uma imersão onde $M$ é uma variedade com conexão riemanniana $\tilde{\nabla}$, sendo $X, Y$ campos locais de vetores em $N$ podemos estendê-los a campos locais $\tilde{X}, \tilde{Y}$ em $M$, respectivamente, e definir a conexão (riemanniana) em $N$ :

$$
\nabla_{X} Y=\left(\tilde{\nabla}_{\tilde{X}} \tilde{Y}\right)^{T}
$$

onde $\left(\tilde{\nabla}_{\tilde{X}} \tilde{Y}\right)^{T}$ denota a componente tangencial de $\tilde{\nabla}_{\tilde{X}} \tilde{Y}$. Esta última coincide com a conexão riemanniana de $N$ com a métrica induzida de $M$.

Com isso, definimos a aplicação bilinear simétrica

$$
B(X, Y)=\tilde{\nabla}_{\tilde{X}} \tilde{Y}-\nabla_{X} Y
$$

Tomando $p \in N$ e $\eta \in \nu_{p} N$, definimos:

$$
H_{\eta}: T_{p} N \times T_{p} N \longrightarrow \mathbb{R}
$$

por

$$
H_{\eta}(X, Y)=\langle B(X, Y), \eta\rangle
$$

a qual é também bilinear simétrica.

Definição 3.5 (Segunda Forma Fundamental). A forma quadrática

$$
I I_{\eta}(X)=H_{\eta}(X, X)
$$

é chamada segunda forma fundamental de $f$ em $p$ segundo o vetor normal $\eta$ (veja a observação 3.4 acima).

Definição 3.6 (Imersão totalmente geodésica). Uma imersão $f: N \longrightarrow M$ é geodésica em $p \in M$ se $I I_{\eta}$ é identicamente nula para todo $\eta \in \nu_{p} N$, e totalmente geodésica se $f$ é imersão geodésica para todo $p \in N$.

Proposição 3.7. $f$ é imersão totalmente geodésica se, e somente se, toda geodésica em $N$ é também geodésica em $M$.

\subsection{A Prova da Conjectura}

Considere uma ação isométrica própria $G \times M \longrightarrow M$. Tomando um ponto regular $q \in M$, podemos considerar uma bola normal $\mathcal{B}_{\varepsilon}(q)$, que admite uma distribuição horizontal integrável $\mathcal{H}$. Logo, existe uma folha $H$ na bola normal de maneira que $H \perp\{G(x)\}_{x \in \mathcal{B}_{\varepsilon}(q)}$. Diremos que $\sigma$ é uma seção regular se $\sigma$ é aberto de uma folha de $H$. 
Teorema 3.8. Seja $M$ uma variedade riemanniana completa e $G$ um grupo que age propriamente por isometrias em $M$. Se a distribuição de espaços normais às órbitas regulares for integrável, então existe uma seção imersa totalmente geodésica para a ação de $G$ a qual encontra todas as órbitas e sempre perpendicularmente.

Na observação a seguir, apresentamos uma definição da variedade grassimaniana por meio do conceito de ação que estudamos no capítulo 2. Uma outra forma muito interessante de definir é identificando as bases ortogonais de um mesmo k-plano, o que pode ser verificado em [B], pg. 62.

Observação 3.9 (Grassmaniano de k-planos em $\mathbb{R}^{n}$ ). Seja $G_{k}\left(\mathbb{R}^{n}\right), k \leq n$ o conjunto dos subespaços vetoriais k-dimensionais de $\mathbb{R}^{n}$. Para dar uma estrutura de variedade a este conjunto, consideramos a ação natural:

$$
\mu: \mathcal{O}_{n} \times G_{k}\left(\mathbb{R}^{n}\right) \longrightarrow G_{k}\left(\mathbb{R}^{n}\right)
$$

Como na Proposição 2.36 do capítulo 2, temos a comutatividade do diagrama:

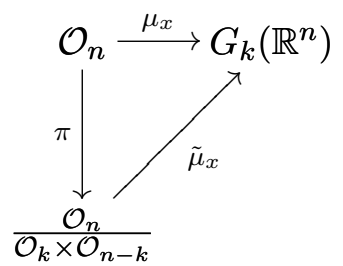

o que dá a $G_{k}\left(\mathbb{R}^{n}\right)$ estrutura de variedade. Esta é chamada Variedade de Grassman.

No caso de uma variedade $M$, vamos definir $G_{k}(M):=\cup_{p} G_{k}\left(T_{p} M\right)$

Para facilitar o entendimento do texto, adotamos agora uma convenção: dada uma ação isométrica própria $\mu: G \times M \longrightarrow M$, vamos considerar $\mathcal{F}=\{G(x)\}_{x \in M}$ a folheação de $M$ dada pelas órbitas regulares da ação $\mu$. Quando denotarmos $\mathcal{F}$, ficará entendido que tratamos da folheação dada pelas órbitas regulares.

No próximo exemplo pretendemos dar uma visão simples da folheação determinada pelas órbitas regulares de uma ação isométrica própria. Na Proposição 3.11 bem como na observação que a segue, explicamos como ocorre a ação induzida.

Exemplo 3.10. Considere $M=S^{2} \times \mathbb{R}$, e $G=S O(2) \times \mathbb{R}$. Tomando $q$ o pólo norte da esfera, temos que as órbitas $G(x)$, para $x \neq q$ e de sua antípoda, são difeomorfas a $S^{1} \times \mathbb{R}$ e ortogonais a $S^{2} \times 0$, e $G(q)$ é o próprio eixo $0 z$. Assim, $S_{q}=\mathcal{U} \times\{0\}$ onde $\mathcal{U}$ é uma vizinhança de $q$ em $S^{2}$ e $G_{q}=S O(2)$. Neste caso temos que, $\left.\mathcal{F}\right|_{S_{p}}$ são os paralelos da esfera e $\tilde{\mathcal{F}}=\{S O(2) \cdot \vec{X}\}_{\vec{X} \in \mathbb{R}^{2}}$ são circunferências centradas na origem em $T_{q} S_{q}$. Note que temos um difeomorfismo entre $\tilde{\mathcal{F}}$ e $\left.\mathcal{F}\right|_{S_{q}}$.

Enunciamos a seguir um resultado importante no processo de demonstração desse teorema. Como não apresentamos sua demonstração, indicamos que pode ser conferida em [P]. 
Proposição 3.11 (Boualem). Seja $M$ uma variedade riemanniana completa, $G \times M \longrightarrow M$ uma ação isométrica própria, $S_{q}$ slice em $q$ de raio $\varepsilon$ e $\sigma$ uma seção regular contida na bola normal $\mathcal{B}_{\varepsilon}(q)$. Suponha que $\sigma$ insersecta $S_{q}$. Então:

a) Existe um subespaço $V \subset T_{q} S_{q}$ de maneira que

$$
\sigma \subset \mathcal{D}_{\varepsilon}(q):=\exp _{q}(V \cap \mathcal{U})
$$

onde $\mathcal{U}=\mathcal{B}_{\varepsilon}(0) \subset T_{q} S_{q}$.

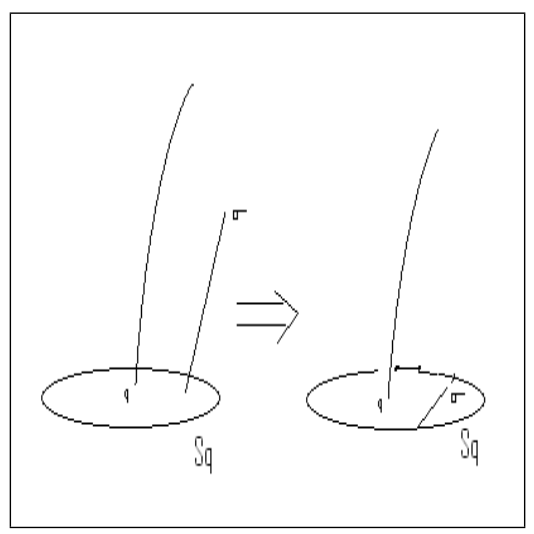

Figura 3.1: Proposição de Boualem

Observação 3.12. Quanto à Proposição anterior vale lembrar que pela definição de slice temos uma ação $\mu: G_{q} \times S_{q} \longrightarrow S_{q}$ o que nos dá uma folheação $\left.\mathcal{F}\right|_{S_{q}}=\left\{G_{q}(y)\right\}_{y \in S_{q}}$. Isto induz uma ação

$$
\begin{gathered}
\tilde{\mu}: G_{q} \times T_{q} S_{q} \longrightarrow T_{q} S_{q} \\
(g, X) \longmapsto d\left(\mu^{g}\right)_{q} X
\end{gathered}
$$

e então temos uma folheação $\tilde{\mathcal{F}}=\left\{G_{q}(X)\right\}_{X \in T_{q} S_{q}}$. Desta forma, $\exp _{q}^{-1}$ é um difeomorfismo entre $\left.\mathcal{F}\right|_{S_{q}}$ e $\tilde{\mathcal{F}}$.

Proposição 3.13. Se $\gamma$ é uma geodésica em $M$ ortogonal a uma órbita regular, então o conjunto dos pontos singulares é isolado em $\gamma$.

Demonstração: Considere primeiro o caso linear onde $G=\mathcal{O}_{n}$ e temos a ação natural:

$$
\begin{gathered}
\mathcal{O}_{n} \times \mathbb{R}^{n} \longrightarrow \mathbb{R}^{n} \\
(g, x) \longmapsto g \cdot x
\end{gathered}
$$

Neste caso, $\overrightarrow{0}$ é órbita singular. Vamos verificar que, se $G(\vec{X})$ é regular, então $G(\lambda \vec{X})$ é regular para qualquer $\lambda \neq 0$.

De fato, $G_{\lambda X}=G_{X}$ e como $G(X)=G / G_{X}$, temos que $\operatorname{dim} G(X)=\operatorname{dim} G(\lambda X)$. Logo, $G(\lambda X)$ é regular. 
O caso geral segue do caso linear discutido acima junto com as propriedades de representação isotrópica.

Lema 3.14. Seja $S_{q}$ slice em $q \in M$ e $\tilde{\mathcal{F}}$ conforme definida na Proposição 3.11. Considere $V$ um subespaço ortogonal às folhas de $\tilde{\mathcal{F}}$. Então:

a) $O$ conjunto dos pontos regulares de $\tilde{\mathcal{F}}$ é aberto e denso em $V \cap \mathcal{U}$, o qual é ortogonal às folhas de $\tilde{\mathcal{F}}$.

b) $O$ conjunto dos pontos regulares de $\mathcal{F}$ é aberto e denso em $\mathcal{D}_{\varepsilon}(q):=\exp _{q}(V \cap \mathcal{U})$.

c) A subvariedade $\mathcal{D}_{\varepsilon}(q)$ é ortogonal às folhas de $\mathcal{F}$

d) $\mathcal{D}_{\varepsilon}(q)$ é uma subvariedade totalmente geodésica.

\section{Demonstração:}

a) Vamos separar a prova em três partes:

i) O conjunto é denso.

Seja $z \in V \cap \mathcal{U}$. Precisamos mostrar que, tão próximo quanto se queira de $z$, é possível encontrar pontos regulares.

De fato, tome um ponto regular $x \in V \cap \mathcal{U}$ (o que é possível pois tomamos $V$ ortogonal a uma folha regular). Considere $\gamma$ a reta em $V \cap \mathcal{U}$ ligando $x$ a $z$. Como $\gamma \subset V$ e $V \perp G(x)$, temos que $\gamma \perp G(x)$. Já que $x$ é regular, segue da Proposição 3.13 que os pontos singulares ao longo de $\gamma$ são isolados. Em particular, como $z \in \gamma$, é possível encontrar pontos regulares tão próximo de $z$ quanto se queira.

ii) O conjunto é aberto.

Seja $z \in V \cap \mathcal{U}$ ponto regular. Mostremos que existe uma vizinhança de $z$ formada por pontos regulares. Como $G(z)$ é regular, as órbitas próximas a $G(z)$ são também regulares. Como $\operatorname{dim}(V)=\operatorname{codim}(G(z))$, tais órbitas intersectam $V$.

iii) $O$ conjunto dos pontos regulares em $V \cap \mathcal{U}$ é ortogonal às órbitas.

Seja $z \in V \cap \mathcal{U}$ um ponto regular e construa $\ell_{1}, \ldots, \ell_{k}:[0,1] \longrightarrow V$ como segue:

$$
\begin{aligned}
& \ell_{i}(0) \in \tilde{\sigma} \\
& \ell_{i}(1)=z \\
& \left\{\ell^{\prime}(1)\right\} \text { é base de } T_{z} V
\end{aligned}
$$

Temos que $\ell_{i} \perp G\left(\ell_{i}(0)\right)$, de onde temos que $\ell_{i} \perp G(z), \forall i$. Consequentemente, $G(z) \perp V$.

b) Sendo $A$ o conjunto dos pontos regulares de $\tilde{\mathcal{F}}$, temos que $\exp _{q}(A)$ é aberto e denso, visto que $\exp _{q}$ é suave.

c) Tome $y \in \mathcal{D}_{\varepsilon}(q)$ ponto regular e $\sigma_{y}$ seção regular contendo $y$. Provemos que $\sigma_{y} \subset \mathcal{D}_{\varepsilon}(q)$.

Seja $\tilde{\sigma_{y}}:=\exp _{q}^{-1}\left(\sigma_{y}\right)$, da Proposição 3.11 temos que $\tilde{\sigma_{y}}$ é seção regular de $\tilde{\mathcal{F}}$. Pelo item $(a)$, $\tilde{\sigma_{y}} \subset \tilde{V} \cap \mathcal{U}$ já que $G(\tilde{y}) \perp V$. 
d) Segue diretamente de $c$ ) e do fato que pontos regulares são densos em $\mathcal{D}_{\varepsilon}(q)$.

Proposição 3.15. Seja $q \in M$ e $\mathcal{D}_{\varepsilon}(q)=\exp _{q}(V \cap \mathcal{U})$. Seja $\mathcal{B}_{\delta}(q)$ bola normal de raio $\delta$ em $M$. Defina

$$
\mathcal{D}_{\delta}(q):=\left\{\exp _{q}(v): v \in T_{q} \mathcal{D}_{\varepsilon}(q),\|v\|<\delta\right\}
$$

Então:

a) O conjunto dos pontos regulares de $\mathcal{F}$ é aberto e denso em $\mathcal{D}_{\delta}(q)$.

b) $\mathcal{D}_{\delta}(q)$ é ortogonal às folhas de $\mathcal{F}$.

c) $\mathcal{D}_{\delta}(q)$ é totalmente geodésica.

Observação 3.16. Vale ressaltar que $\delta$ da Proposição 3.15 é frequentemente maior que $\varepsilon$ do lema 3.14

Demonstração: Seja $\xi \in T_{q} \mathcal{D}_{\varepsilon}(q)$ tal que $\gamma(t)=\exp _{q}(t \xi)$ tem pontos regulares $(0 \leq t \leq 1)$. Da Proposição 3.13, os pontos singulares são isolados em $\gamma$. Como $\gamma$ é compacto, podemos cobrir $\gamma$ com um número finito de slices centrados em $\gamma\left(t_{i}\right)$ tal que $\gamma\left(t_{i}\right)$ é o único ponto singular possível em $\gamma \cap S_{\gamma\left(t_{i}\right)}$.

Construa $T=\cup_{i} \mathcal{D}_{\varepsilon}\left(\gamma\left(t_{i}\right)\right)$ conforme lema 3.14.

Como $\exp _{q}$ é difeomorfismo e $T$ é totalmente geodésica, podemos encontrar vizinhança $\mathcal{W}$ de $\xi$ em $T_{q} \mathcal{D}_{\varepsilon}(q)$ e definir vizinhança de $\gamma$ em $T$ por $N:=\exp _{q}(\mathcal{W})$. Além disso, $N \subset \mathcal{D}_{\delta}(q)$. Assim, temos vizinhança $N$ de $\gamma \operatorname{em~} \mathcal{D}_{\delta}(q)$ satisfazendo os itens (a) a (c). Da arbitrariedade da escolha de $\xi$ segue o resultado.

Agora que enunciamos alguns resultados necessários, vamos efetuar a prova da conjectura de Palais-Terng.

\subsubsection{Demonstração do Teorema 3.8}

Seja $q \in M$ e

$$
\mathcal{D}_{r_{1}}(q):=\left\{\exp _{q}(v): v \in T_{q} \mathcal{D}_{\varepsilon}(q) \text { e }\|v\| \leq r_{1}\right\}
$$

onde $r_{1}>0$. Como $\mathcal{D}_{r_{1}}(q)$ é compacto, é possível encontrar uma vizinhança compacta $\tilde{\mathcal{U}}$ de $\mathcal{D}_{r_{1}}(q)$ e $\delta>0$ (a saber, o delta de Lebesgue) tal que, para todo $x \in \tilde{\mathcal{U}}, \mathcal{B}_{\delta}(x)$ é bola normal. Seja $\gamma$ geodésica de comprimento $r_{1}$ tal que $\gamma^{\prime}(0) \in T_{q} \mathcal{D}_{\varepsilon}(q)$. Note que podemos estender $\mathcal{D}_{\varepsilon}(q)$ ao longo da geodésica $\gamma$ para uma subvariedade (não necessariamente completa) satisfazendo a Proposição 3.15 , por meio de colagem de discos totalmente geodésicos de raio $\delta / 2$. 


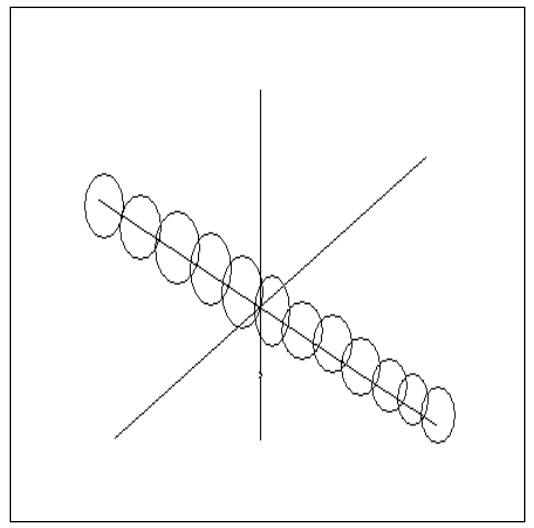

Figura 3.2: Colagem de discos ao longo da geodésica

Explicando melhor, considere inicialmente $\mathcal{D}_{\delta / 2}(q)$, o qual satisfaz os itens $(a)-(c)$ da Proposição 3.15. Defina o segundo disco como

$$
\mathcal{D}_{\delta / 2}(\gamma(\delta / 4)):=\left\{\exp _{\gamma(\delta / 4)}(v): v \in T_{\gamma(\delta / 4)} \mathcal{D}_{\delta / 2}(q) \text { e }\|v\| \leq \delta / 2\right\}
$$

Este conjunto também satisfaz os itens $(a)-(c)$ da Proposição 3.15. Continuando esse processo, obtemos discos por indução e estendemos $\mathcal{D}_{\varepsilon}(q)$ ao longo de $\gamma$.

Seja $\Sigma_{r_{1}}$ a união das extensões construidas acima ao longo de todas as geodésicas $\gamma$ com comprimento $r_{1}$ tal que $\gamma^{\prime}(0) \in T_{q} \mathcal{D}_{\varepsilon}(q)$. Usaremos o lema abaixo:

Lema 3.17. $\Sigma_{r_{1}}$ pode ser coberto por um número finito de discos $\mathcal{D}_{i}$ 's (de raio $\delta$ ) satisfazendo os itens $(a)-(c)$ da Proposição 3.15.

Demonstração: Vamos provar a seguinte afirmação necessária para o lema:

Afirmação 3.18. Seja $x \in \mathcal{D}_{r_{1}}(q)$. Então, $\mathcal{D}_{r_{1}}(q) \cap \mathcal{B}_{\delta / 4}(x)$ pertence a um número finito de discos $\mathcal{D}_{i}$ 's de raio $\delta / 2$ satisfazendo os itens $(a)-(c)$ da Proposição 3.15 .

Prova: Seja

$$
K=\left\{v \in T_{q} \mathcal{D}_{\varepsilon}(q): \exp _{q}(v) \in \overline{\mathcal{B}}_{\delta / 4}(x) \text { e }\|v\| \leq r_{1}\right\}
$$

Note que $K$ é compacto. Agora tome $v_{0} \in K$. Conforme apresentado acima, podemos estender $\mathcal{D}_{\varepsilon}(q)$ ao longo de $\exp _{p}\left(t v_{0}\right)$ para uma subvariedade totalmente geodésica $T$. Como exp é contínua, podemos encontrar $\mathcal{W}$ vizinhança de $v_{0}$ tal que $\exp _{q}(t u) \subset T$, para todo $u \in \mathcal{W} \cap K$ e $0<t \leq 1$. Da compacidade de $K$ segue a afirmação.

Para concluir a prova do lema, vamos cobrir $\mathcal{D}_{r_{1}}(q)$ com um número finito de bolas $\mathcal{B}_{\delta / 4}^{i}$. Pela afirmação, deduzimos que $\mathcal{D}_{r_{1}}(q)$ está contida em uma união finita de discos $\mathcal{D}_{\delta / 2}\left(p_{i}\right)$ satisfazendo (a) - (c) da Proposição 3.15. Assim, conforme definimos $\Sigma_{r_{1}}$, concluimos que para todo $x \in \Sigma_{r_{1}}$, existe $i_{0}$ tal que $x \in \mathcal{D}_{\delta}\left(p_{i_{0}}\right)$. 
Dando continuidade à prova do teorema, vamos provar que $\Sigma_{r_{1}}$ é subvariedade imersa em $M$. Considere $\left(G^{k}(M), M, \pi\right)$ o k-grassmaniano de $M$ e defina $\mathcal{D}_{i}^{*} \subset G^{k}(M)$ por

$$
\mathcal{D}_{i}^{*}:=\left\{T_{x} D_{i}: x \in D_{i}\right\}
$$

e $\Sigma_{r_{1}}^{*} \subset G^{k}(M)$ por

$$
\Sigma_{r_{1}}^{*}=\left\{T_{x} D_{i}\right\}_{x \in \tilde{\Sigma}_{r 1}}
$$

$D_{i}^{*}$ é imersa em $G^{k}(M)$, e se $D_{i}^{*}$ e $D_{j}^{*}$ têm um ponto em comum então eles coincidem numa vizinhança desse ponto. Como $\Sigma_{r^{1}}^{*}$ pode ser recoberto por um número finito de $D_{i}^{*}$, vemos que $\Sigma_{r^{1}}^{*}$ é mergulhada em $G^{k}(M)$. Concluimos que $\Sigma_{r^{1}}$ é imerso sendo $\Sigma_{r^{1}}=\prod \Sigma_{r^{1}}^{*}$.

Para concluir a prova, tome uma sequência $r_{1}<r_{2}<\ldots<r_{n} \longrightarrow \infty$ e construa $\Sigma_{r^{i}}$ e $\Sigma_{r^{i}}^{*}$ como acima. Podemos mostrar assim que $\Sigma_{r^{i}}^{*}$ é mergulhada e $\Sigma_{r^{i}}$ imersa. Finalmente, defina

$$
\Sigma^{*}:=\cup_{r_{i}} \Sigma_{r^{i}}^{*}
$$

Temos que $\Sigma^{*}$ é uma subvariedade e que $\Sigma:=\cup_{r_{i}} \Sigma_{r_{1}}$ é imersa para $\Sigma=\prod \Sigma^{*}$. Usando o Teorema de Hopf-Rinow (teorema 1.84) temos que $\Sigma$ é completa. 


\section{Referências Bibliográficas}

[A] Alexandrino, M. M., Proofs of Conjectures about Singular Riemannian Foliations. Geom Dedicata (2006) 119:219-234. Springer.

[B] Boothby, W. M., An Introduction to Differentiable Manifolds and Riemannian Geometry Revised Second Edition, San Diego: Academic Press, 2003.

[CL] Camacho, C., Lins Neto, A., Teoria Geométrica das Folheações, Rio de Janeiro: IMPA, 1979.

[dC1] Carmo, M. P. do, Geometria Diferencial de Curvas e Superfícies, Rio de Janeiro: SBM, 2005.

[dC2] Carmo, M. P. do, Geometria Riemanniana, Terceira Edição, Rio de Janeiro: IMPA, 2005.

[DK] Duistermaat, J., Kolk, J., Lie Groups, Berlin: Springer-Verlag, 2000.

[F] Fegan, H. D., Introduction to Compact Lie Groups, Series in Pure Mathematics, 13. World Scientific Publishing Co., Inc., River Edge, NJ, 1991.

[HOL] Heintze, E.; Liu, X.; Olmos, C. Isoparametric Submanifolds and a Chevalley-Type Restriction Theorem.

[KN] Kobayashi, S., Nomizu, K., Foundations of Differential Geometry, Vol. I. John Wiley Sons Inc. 1996.

[L1] Lima, E. L., Espaços Métricos, Quarta Edição, Rio de Janeiro: IMPA, 2007.

[L2] Lima, E. L., Grupo Fundamental e Espaços de Recobrimento, Terceira Edição, Rio de Janeiro: IMPA, 2006.

[P] Pineros, D. A. C., Sobre as folheações e o teorema de slice para folheações riemannianas singulares com seções, Dissertação de Mestrado, São Paulo: IME/USP, 2008.

[PT] Palais, R.S, Terng, C-L, Critical Point Theory and Submanifold Geometry, Lectures Notes in Mathematics 1353: Springer Verlag, 1988.

[S] Spivak, M., A Comprehensive Introduction to Differential Geometry, vol. 1, Boston: Publish or Perish, [1970 - 1975]. 